\title{
Integration time adjusted completeness
}

\author{
Dean Keithly $\oplus_{,}^{\text {a,b, }, *}$ Dmitry Savransky $\oplus^{\text {a,b }}$ and Corey Spohn $\oplus^{\mathrm{a}, \mathrm{b}}$ \\ ${ }^{a}$ Cornell University, Sibley School of Mechanical and Aerospace Engineering, \\ Ithaca, New York, United States \\ ${ }^{b}$ Cornell University, Carl Sagan Institute, Ithaca, New York, United States
}

\begin{abstract}
Future, large-scale, exoplanet direct-imaging missions will be capable of discovering and characterizing Earth-like exoplanets. These mission designs can be evaluated using completeness, the fraction of planets from some population that are detectable by a telescope at an arbitrary observation time. However, the original formulation of completeness uses instrument visibility limits and ignores additional integration time and planetary motion constraints. Some of the sampled planets used to calculate completeness may transit in and out of an instrument's geometric and photometric visibility limits while they are being observed, thereby causing the integration time agnostic calculation to overestimate completeness. We present a method for calculating completeness that accounts for the fraction of planets that leave the visibility limits of the telescope during the integration time period. We define completeness using the aggregate fraction of an orbital period during which planets are detectable, calculated using the specific times that planets enter and leave an instrument's visibility limits and the integration time. To perform this calculation, we derive analytical methods for finding the planet-star projected separation extrema, times past periastron that these extrema occur, and times past periastron that the planet-star projected separation intersects a specific separation circle. We also provide efficient numerical methods for calculating the planet-star difference in magnitude extrema and times past periastron corresponding to specific values $\Delta$ mag. Our integration time adjusted completeness shows that, for a planned star observation at $25 \mathrm{pc}$ with 1-day and 5-day integration times, integration time adjusted completeness of Earth-like planets is reduced by $1 \%$ and $5 \%$ from the integration time agnostic completeness, respectively. Integration time adjusted completeness calculated in this manner also provides a computationally inexpensive method for finding dynamic completeness - the completeness change on subsequent observations. (C) The Authors. Published by SPIE under a Creative Commons Attribution 4.0 Unported License. Distribution or reproduction of this work in whole or in part requires full attribution of the original publication, including its DOI. [DOI: 10 .1117/1.JATIS.7.3.037002]
\end{abstract}

Keywords: exoplanets; completeness; dynamic completeness; direct imaging; modeling; optimization.

Paper 21035 received Mar. 30, 2021; accepted for publication Aug. 16, 2021; published online Sep. 1, 2021.

\section{Introduction}

Direct imaging blind search mission schedules can be optimized ${ }^{1}$ by maximizing completeness ${ }^{2}$ the fraction of exoplanets from an assumed planet population that are detectable by a particular instrument at an arbitrary observation time. Completeness is typically parameterized by a limiting planet-star brightness difference $\left(\Delta \mathrm{mag}_{\text {lim }}\right)$, the inner working angle (IWA) of the instrument, and the outer working angle (OWA) of the instrument. The original Monte Carlo approach developed by Brown ${ }^{2}$ involved creating a cloud of synthetic planets by sampling the underlying Keplerian orbital elements (KOE) and physical parameters of a planet population and determining the fraction of those individually simulated planets within the visible limits of the instrument. Multiplying completeness by the exoplanet occurrence rate gives the expected exoplanet yield for observing a given star. Although completeness is a good metric for predicting instrument performance, the calculation described above only captures an instant in time and

*Address all correspondence to Dean Keithly, drk94@cornell.edu 
does not include whether the time that a planet is within the visible region of the telescope is sufficient to actually make a detection.

The original method of calculating completeness was developed by Brown. ${ }^{2}$ Our adaptation of this method in EXOSIMS ${ }^{3}$ is extensively outlined in Ref. 1 and involves randomly generating planet KOE, planet geometric albedo $(p)$, and planetary radius $(R)$ sampled from NASA's Exoplanet Program Analysis Group Study Analysis Group 13 (SAG13) ${ }^{1,4,5}$ probability density functions, generating a finely binned 2D histogram of $\Delta$ mag versus $s$ (planet-star separation), and fitting a 2D spline to the histogram bins. In all current methods, completeness is calculated as the double integral of $s$ and $\Delta$ mag over the joint probability density function as in Eq. (17) of Ref. 1 and Eq. (7) in Ref. 6. The limits of integration define a detectable planet as one in which $\Delta$ mag $<\Delta \operatorname{mag}_{\text {lim }}$ and $d_{i}$ IWA $<s<d_{i}$ OWA, where $d_{i}$ is the distance of the host star from the spacecraft. While the $\Delta \operatorname{mag}_{\text {lim }}$ in this paper is used to describe a general upper limit of integration for calculating completeness, this $\Delta \operatorname{mag}_{\text {lim }}$ can be formulated as a function of integration time as in Eq. (12) of Ref. 1 by making assumptions about a multitude of instrument parameters and external noise sources. This approach, as conventionally implemented, is a good estimator for completeness, ${ }^{1}$ but it does not take planet motion in time into account.

Searches like the Gemini Planet Imager and Nancy Grace Roman Space Telescope (Roman) Coronagraphic Instrument (CGI) ${ }^{7}$ are sensitive to larger planets with larger planet-star separations and longer orbital periods. ${ }^{1}$ They make use of Brown completeness to plan blind searches. However, future telescope concepts like the Habitable Exoplanet Observatory $(\mathrm{HabEx})^{8}$ and the Large UV/Optical/IR Surveyor (LUVOIR) ${ }^{9}$ seek to find smaller Earth-like exoplanets, with shorter orbital periods around stars farther away. Their search criteria will result in Brown completeness based yield overestimations due to planet motion out of instrument visibility limits. Figure 1 shows a schematic of a direct imaging observation; it demonstrates how a planet can be within the photometric and geometric visibility limits of the instrument and still not be detected. The red regions of the planet's orbit indicate where the planet is within the visible limits but not detectable because the planet will move out of the region in less time than it takes to detect the planet [case (b) in this figure]. Since there will be some Earth-like exoplanets that are counted toward the completeness score but are not actually within the visibility constraints of the

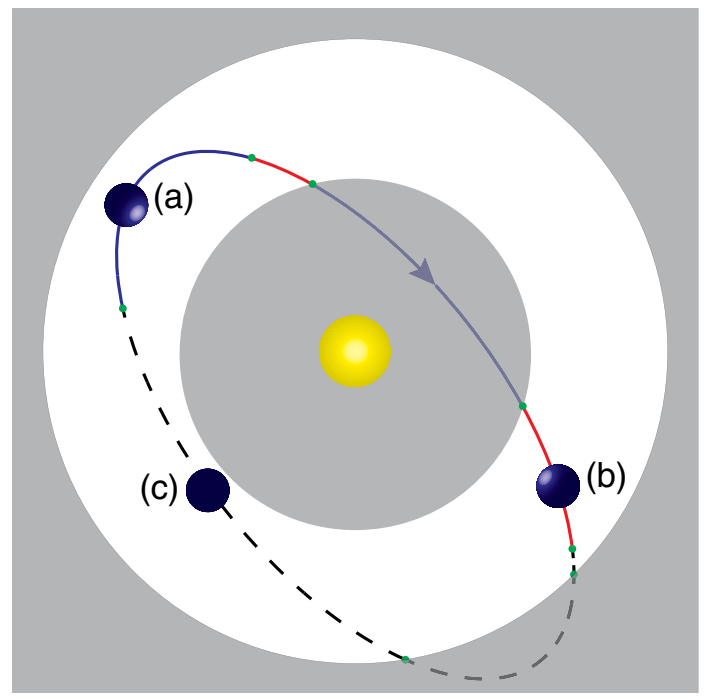

Fig. 1 Schematic of a direct imaging observation. The line represents the projection of a planet's orbit about its parent star (yellow) into the plane of the sky as seen by a distant observer. The arrow indicates the planet's direction of motion. Blue portions of the orbit (a) indicate times when the planet is detectable. Red portions of the orbit (b) indicate times when the planet is within the visibility limits of the instrument, but it is not detectable due to integration time constraints. The dashed portions of the orbit (c) indicate times when the planet is too faint to be observed. The shaded gray regions represent the projected inner and OWAs of the instrument. The planet is unobservable on portions of the orbit intersecting these gray regions. Green dots indicate transitions in and out of instrument visibility limits. 
instrument long enough to be directly imaged, we need a new method that only counts targets within the instrument visibility long enough to be observed or characterized.

Figure 2 demonstrates this phenomenon in the separation versus $\Delta$ mag phase space commonly used to define instrument contrast curves and completeness. Figure 2(b) shows a shortperiod, fast-moving, Mars-like planet that transitions into and out of the assumed instrument's visible limits before it can be detected. Figure 2(c) shows the effect for a Uranus-like planet that crosses through and exits the visible region in less time than it takes for it to be detected. In these cases, Brown's completeness calculation would include simulated planets in these regions, thus overestimating the overall detection yield. Additionally, Fig. 2(d) shows that the last possible moment a Neptune-like planet could be imaged before it leaves the visible limits of the telescope. This demonstrates a portion of the orbit where the planet is within the visible limits of the telescope but is not detectable.

This paper presents a method, implemented in the EXOSIMS modeling software, ${ }^{3}$ for calculating integration time adjusted completeness that allows us to investigate exactly how much the original completeness definition overestimates planet yields. EXOSIMS is an exoplanet direct imaging mission modeling software used to simulate the Roman $\mathrm{CGI}^{7}$ and future mission concepts such as $\mathrm{HabEx}^{8}$ and LUVOIR. ${ }^{9}$ EXOSIMS simulates populations of planets, observatories, instruments, and underlying dynamics to create full, end-to-end mission simulations. We derive distributions of potential mission yield from ensembles of these mission simulations. EXOSIMS utilizes completeness as a heuristic for target selection and observation scheduling, ${ }^{1}$
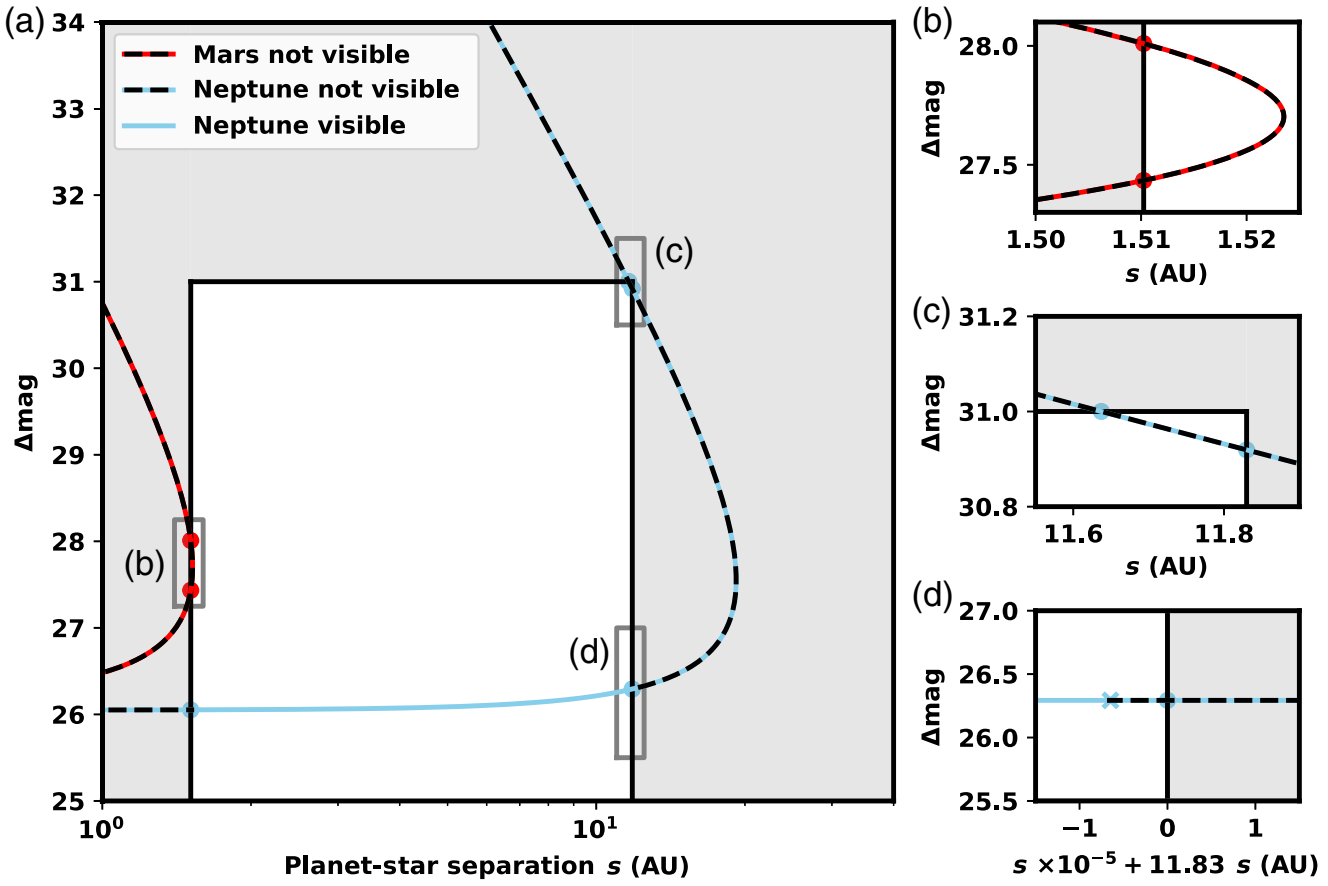

Fig. 2 The $\Delta$ mag versus s curve for Neptune (blue) and Mars (red). The instrument's visible region (white) is bounded by 0.066 and 0.517 arc sec, and $\Delta$ mag $=31$ at $d_{i}=22.87 \mathrm{pc}$ (we selected instrument parameters to make a demonstration on solar system planets about a Sun-like star, but any instrument will have planets with similar effects). Any planet in the grayed region is not visible by the instrument. Dots indicate calculated intersection points between the planet and the visible region bounds. The black dashed portions of planet orbits indicate portions of the orbit where the planet is not detectable. (b) A portion of Mars's orbit where the planet enters and exits the visible limits of the instrument in less time than the required integration time. ${ }^{1}$ (c) A portion of Neptune's orbit where the planet enters and exits the visible region of the instrument in less time than the required integration time. (d) Neptune's detectable region adjusted for integration time. If the observation of Neptune in (d) is started when it is left of the blue $\times$ and in the visible region, then it will be detected; if it is started right of the blue $\times$, the planet will not be detected because the required integration time is larger than the time the planet will be in the visible region of the instrument. 
implementing both Brown's Monte Carlo approach to completeness ${ }^{2}$ as well as the analytical completeness formulation from Ref. 6. However, EXOSIMS determines whether actual detections occur by evaluating the effective signal-to-noise ratio (SNR) of individual simulated observations on simulated planets. Keithly et al. ${ }^{1}$ discussed the full optimization process for a singlevisit blind search and showed that the ideal yield from an EXOSIMS simulation ensemble (when mission constraints are discounted) for a limited blind search mission of Roman is equivalent to the yield calculated via Brown Completeness.

Comparisons between Brown completeness-based exoplanet yield estimations and Monte Carlo of full mission simulations have been done in Ref. 10 and the Standard Definitions and Evaluation Team Final Report. ${ }^{11}$ The yields calculated by completeness are higher than the average yield from a Monte Carlo of full missions simulated in EXOSIMS as noted in Table 6 of Ref. 11. These works attribute the difference in yields to the additional mission constraints captured by the full mission simulation approach, inefficiencies in the optimization algorithms, and inefficiencies in the scheduling algorithms implemented in EXOSIMS. ${ }^{12}$ The unmentioned assumption is that Brown completeness-based yield estimates are accurate, but this paper demonstrates how the omission of planet motion and integration time reduces the resulting completeness yield estimates.

To know the amount of time that an individual planet spends within the observable limits of an instrument, we need to calculate the times in a planet's orbit when a planet enters or exits these limits. This means that we need methods for calculating when a planet has a given planetstar separation and $\Delta$ mag. Once we know all of the times when a planet enters or exits an instrument's visibility limits, we know the fraction of time that the planet is able to be detected with that instrument. Averaging over these visibility fractions for a large number of samples evaluates completeness in a fundamentally different way from its original formulation. These same methods also allow for the evaluation of integration time adjusted completeness as well as a new method of calculating dynamic completeness. ${ }^{13}$

Section 2 presents the derivation of this chain of calculations and discusses practical aspects of their implementation. Section 3 provides detailed validation of the methods and presents results of the various calculations enabled by them. Finally, in Section 4, we discuss various aspects of the algorithms and results, and we lay out future applications for this methodology.

\section{Methods}

In this section, we present our detailed process for calculating integration time adjusted completeness. We do this by finding the time windows in which a planet is within the separation and $\Delta$ mag visibility limits of an instrument, discounting each time window by the integration time. The general overview of this process is as follows.

1. Calculate locations of apparent intersections between the projection of the 3D orbit into the plane of the sky and the $s_{\mathrm{WA}}$ circle about the star.

(a) Parameterize the 3D orbit as an ellipse in the plane of the sky.

(b) Formulate the planet-star separation equation and its derivative.

(c) Solve for the magnitude and locations of planet-star separation extrema.

(d) Identify and assign the subset of algebraic solutions that are separation extrema.

(e) Classify the expected number of intersections between the projected ellipse and the $s_{\mathrm{WA}}$ circle.

(f) Solve for intersections between the projection of the orbital ellipse and the $s_{\mathrm{WA}}$ circle.

(g) Identify and assign the subset of algebraic solutions that form intersections.

2. Calculate locations and magnitudes of $\Delta$ mag intersections.

(h) Formulate an expression isolating the $\Delta$ mag and $\nu$ terms and its derivative.

(i) Express this formulation as a polynomial and solve for the algebraic solutions.

(j) Identify and assign the subset of algebraic solutions that are $\Delta$ mag extrema. 
(k) Classify the expected number of $\Delta$ mag and $\Delta \operatorname{mag}_{\text {lim }}$ intersections.

(l) Solve for $\Delta$ mag and $\Delta \operatorname{mag}_{\text {lim }}$ intersections.

(m) Identify and assign the subset of algebraic solutions that form the intersections.

3. Calculate $\nu$ from $\mathrm{X}$ and $\mathrm{Y}$ for each intersection and extrema.

4. Calculate $t$ from $\nu$ of each intersection and extrema.

5. Combine times of $s_{\mathrm{WA}}$ and $\Delta \mathrm{mag}_{\text {lim }}$ intersections to create time windows between intersections.

6. Identify time windows in which the planet is visible or not visible.

7. Calculate integration time adjusted completeness averaging the orbital fraction of time that a planet is visible discounted by the integration time.

The general equations for $\Delta$ mag and $s$ used in these derivations are

$$
\Delta \operatorname{mag}=-2.5 \log _{10}\left(p\left(\frac{R}{\left|\underline{r}_{k / i}\right|}\right)^{2} \Phi(\beta)\right),
$$

and

$$
s=\left\|\underline{r}_{k / i}-\left(\underline{r}_{k / i} \cdot \underline{\hat{r}}_{i / \mathrm{SC}}\right) \underline{\hat{r}}_{i / \mathrm{SC}}\right\|
$$

Here $R$ is the planet radius, $p$ is the geometric albedo of the planet, and $\Phi(\beta)$ is the planet phase function. The other variables are defined in Fig. 3, where $\beta$ is the star-planet-observer angle (also called the phase angle), $\underline{r}_{k / i}$ is the vector from star $i$ to planet $k$, and $\underline{\underline{r}}_{i / \mathrm{SC}}$ is the unit vector from the spacecraft (SC) to the star ( $\underline{\hat{r}}$ is the unit vector of $\underline{r})$. The plane of the sky for a given observation lies in the $\underline{\hat{x}}$ and $\hat{\underline{y}}$ plane, where $\underline{\underline{r}}_{i / \mathrm{SC}}$ defines $\underline{\underline{z}}$ of the target system. For our purposes, the direction of $\underline{\hat{x}}$ is arbitrary, but is typically taken to be a well-defined, inertially fixed direction, such as the ICRS mean equinox or pole direction. Here and throughout this paper, $i$ in a subscript refers to the $i$ 'th target star and $i$ (not subscripted) refers to the orbit inclination.

\subsection{Projected Orbit and Separation Intersection}

In this section, we derive an analytical expression for the true anomaly $(\nu)$ of $s$-orbit intersection points between a circle in the plane of the sky and the projection of a 3D Keplerian orbit on the plane of the sky. A practical example of a circle in the plane of the sky is the projected inner or

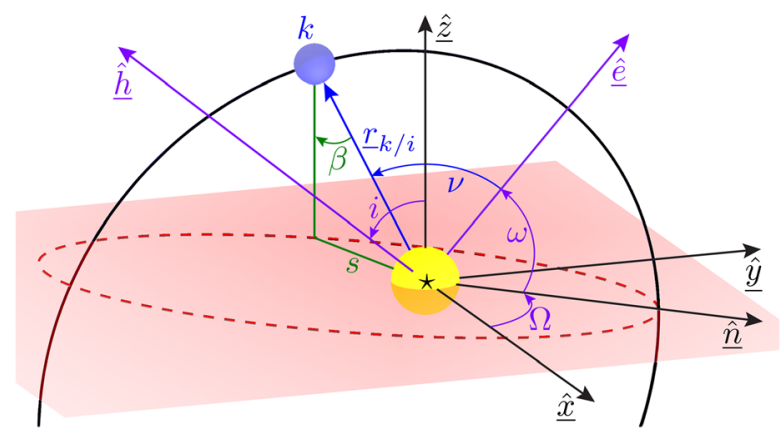

Fig. 3 The orbital path of planet $k$ (black) in a general $X Y Z$ Cartesian coordinate system. $\underline{r}_{k / i}$ (straight blue arrow) describes the location of the planet $k$ (blue circle) relative to star $i$ (yellow circle). The plane of the sky is noted by the translucent red parallelogram entirely in the $\underline{\hat{x}}$ and $\hat{y}$ plane. The dashed ellipse (red) is the projection of the planet orbit (black) onto the plane of the sky. $\underline{\hat{h}}$ is the orbit angular momentum vector. $\underline{\hat{e}}$ is the orbit eccentricity vector. $\underline{\hat{n}}$ is the line of nodes. The star $*$ is generally referred to in subscripts as $*$ and often referred to in subscripts as the $i$ 'th star. This is not to be confused with the variable $i$, which is the inclination of the planet orbit. $\nu$ is the true anomaly of the planet. $\omega$ is the argument of periapsis of the planet. $\Omega$ is the longitude of the ascending node of the planet. 
OWA of the instrument, equal to IWA $d_{i}$ (or OWA $d_{i}$ ) for target star distance $d_{i}$. An orbit defined by KOE takes the shape of an ellipse in the orbital plane. The perpendicular projection of a 3D elliptical orbit onto the plane of the sky is given by the $\underline{\hat{x}}$ and $\hat{\underline{y}}$ components of the 3D orbit. However, the resulting expression is not easily solvable for the true anomalies at intersection points. Instead, we can express the orbit projection in the plane of the sky as another ellipse. This simplifies the difficult intersection problem into analytically solvable subproblems and yields a relatively simple solution to the intersection between a circle in the plane of the sky and the projected orbit in the plane of the sky. Here we include the brief outline of this process, with the full procedure detailed in Appendix B.

1. Project the 3D orbital ellipse into a $2 \mathrm{D}$ projected ellipse and formulate the separation equation.

2. Find the expected number of $s$ intersection points.

3. Calculate $s$ intersection point coordinates.

\subsubsection{Projection of the elliptical orbit}

Given the KOE of a 3D orbit and a plane to project it on, we calculate the semimajor and semiminor axes of the projected orbit ( $a_{p}$ and $b_{p}$, respectively) as well as the angle between the projected semimajor axis and $\underline{\hat{x}}$, which we call $(\theta)$. We first convert the KOE into orbital radius components in the Cartesian $X Y Z$ coordinate system as in Fig. 3:

$$
\begin{aligned}
& X=r(\cos (\Omega) \cos (\omega+\nu)-\sin (\Omega) \sin (\omega+\nu) \cos (i)) \\
& Y=r(\sin (\Omega) \cos (\omega+\nu)+\cos (\Omega) \sin (\omega+\nu) \cos (i)) \\
& Z=r \sin (i) \sin (\omega+\nu),
\end{aligned}
$$

where $r$ is the orbital radius (magnitude of $\underline{r}_{k / i}$ ) given by

$$
r=\frac{a\left(1-e^{2}\right)}{1+e \cos (\nu)} .
$$

$\Omega$ is the longitude of the ascending node, $\omega$ is the argument of periapsis, $\nu$ is the true anomaly, and $e$ is the eccentricity of the planet's orbit.

Figure 4 shows a schematic view of the projection of the orbit onto the plane of the sky. There are two particularly important points associated with the projected orbit ellipse. First, $F$ is the filled focus (star location) of the orbit and retains the same coordinates in the plane of the sky. When observing a star, $F$ is the center of all working angle circles and the origin of the $X Y Z$ coordinate system. Note that in Fig. 4 the projected ellipse is located well below the original 3D ellipse for clarity, but the points $F$ indicated by the orange circle and $F$ indicated by the orange $\times$ are in fact coincident.

Second, $O$ is the geometric center of the 3D orbit and is found most efficiently by averaging the $X Y Z$ locations of the planet at apoapsis and periapsis:

$$
\overline{F O}=\frac{1}{2}\left(\underline{r}_{k / i}(\nu=0)+\underline{r}_{k / i}(\nu=\pi)\right),
$$

where $\overline{F O}$ is the line segment (equivalently Euclidean vector) from $F$ to $O$ and $\underline{r}_{k / i}(\nu)$ is the evaluation of Eq. (3) for the given value of $\nu$. As $\underline{\hat{x}}$ and $\hat{\underline{y}}$ define the plane of the sky, the $X Y Z$ coordinates of the geometric center of the projected ellipse $\left(O^{\prime}\right)$ are given by

$$
O^{\prime}=\langle\overline{F O} \cdot \underline{\hat{x}}, \overline{F O} \cdot \hat{\underline{y}}, 0\rangle .
$$

Appendix $\mathrm{J}$ provides a proof that any generic $3 \mathrm{D}$ ellipse projects to a $2 \mathrm{D}$ ellipse, as shown graphically in Fig. 4. The projection of the semimajor axis and semiminor axis from the 3D orbit onto the 2D plane of the sky form conjugate diameters of the projected ellipse. Any two diameters of an ellipse are conjugate diameters if and only if the tangent line to the ellipse at an 


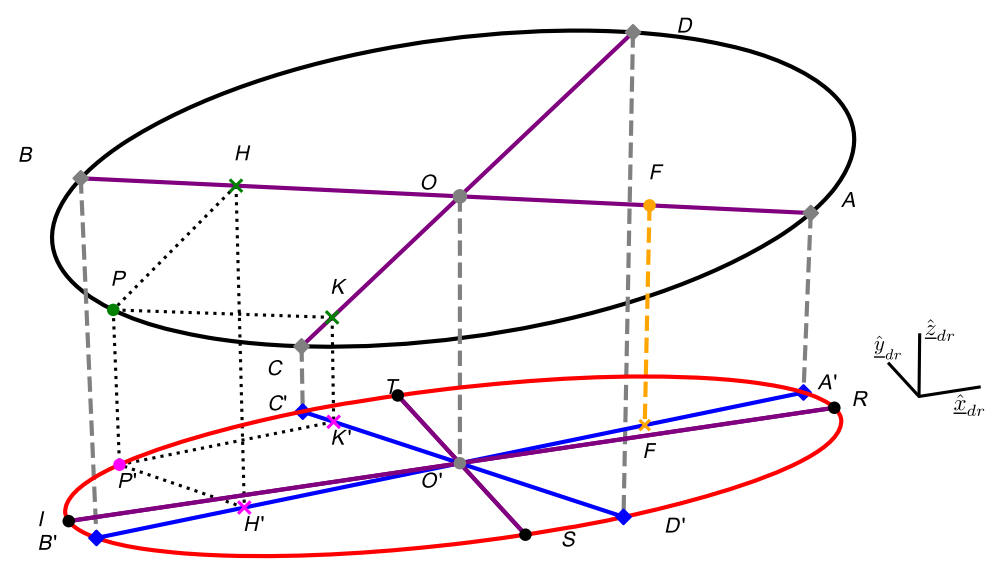

Fig. 4 The original 3D elliptical orbit of a planet (black line) containing points $A, B, C$, and $D$; the gray endpoints of the purple semimajor axis $\overline{A B}$ and semiminor axis $\overline{C D}$. The planet orbits about the star, which is located at focus $F$ (orange circle). The orange dot and orange $\times$ denoted as $F$ are the same point in space, but the red projection is shown as offset from the original orbit for clarity. The projection of the original $3 \mathrm{D}$ elliptical orbit onto the $X Y$ plane is given by the red ellipse containing points $A^{\prime}, B^{\prime}, C^{\prime}$, and $D^{\prime}$ (blue diamonds; perpendicular projections of $A, B, C$, and $D$ ). Point $O$ is the geometric center of the $3 \mathrm{D}$ elliptical orbit and projects to $O^{\prime}$ in the plane of the sky. Point $P$ (green circle) is any arbitrary point along the original 3D ellipse and maps to the semimajor axis and semiminor axis components $H$ and $K$, respectively (green $\times$ ). $P^{\prime}$ (pink circle) is the perpendicular projection of $P$, and $H^{\prime}$ and $K^{\prime}$ (pink $\times$ ) are projections of $H$ and $K$, respectively. The components of these perpendicular projections preserve the ratios of their values to the semimajor and semiminor axes, which, given the equation for an ellipse, can be used to prove the projection of an ellipse is itself an ellipse. The blue lines $\overline{A^{\prime} B^{\prime}}$ and $\overline{C^{\prime} D^{\prime}}$ form conjugate diameters of the red ellipse and are the projection of the semimajor axis and semiminor axis of the original $3 D$ ellipse onto the plane of the sky. These conjugate diameters can then be used to find the semimajor axis and semiminor axis of the red projected ellipse $\overline{I R}$ and $\overline{S T}$, respectively. $\underline{x}_{d r}, \underline{y}_{d r}, \underline{z}_{d r}$ are the components of the derotated reference frame $(d r)$ as defined in the text.

endpoint of one diameter is parallel to the other diameter. ${ }^{14}$ Each pair of conjugate diameters of an ellipse has a corresponding tangent parallelogram, sometimes called a bounding parallelogram. In Fig. 4, the pair of blue lines $\left(\overline{A^{\prime} B^{\prime}}\right.$ and $\left.\overline{C^{\prime} D^{\prime}}\right)$ are specific examples of conjugate diameters. The pair of purple lines $(\overline{I R}$ and $\overline{S T})$ are also examples of conjugate diameters, and they form the semimajor and semiminor axes of the projected ellipse.

The semimajor and semiminor axes of the projected ellipse may be found from any conjugate diameters. We take the conjugate diameters $\overline{A^{\prime} B^{\prime}}$ and $\overline{D^{\prime} C^{\prime}}$ and draw line $\overline{Q Q^{\prime}}$ through $B^{\prime}$, perpendicular to $\overline{D^{\prime} C^{\prime}}$ as shown by the gray line in Fig. 5. Points $Q$ and $Q^{\prime}$ are chosen such that $\overline{B^{\prime} Q}=\overline{B^{\prime} Q^{\prime}}=\overline{O^{\prime} D^{\prime}}$. The principal axes $\overline{I R}$ and $\overline{S T}$ lie on the bisectors of the angles formed by lines $\overline{O^{\prime} Q}$ and $\overline{O^{\prime} Q^{\prime}}$. From this construction, we calculate the projected ellipse semimajor axis, semiminor axis, and angular offset of the semimajor axis from $\underline{\hat{x}}^{16} \overline{I R}$ and $\overline{S T}$ are given by

$$
\begin{aligned}
& \overline{I R}=\overline{O^{\prime} Q^{\prime}}+\overline{O^{\prime} Q}, \\
& \overline{T S}=\overline{O^{\prime} Q^{\prime}}-\overline{O^{\prime} Q} .
\end{aligned}
$$

Defining the angle between $\overline{O^{\prime} B^{\prime}}$ and $\overline{O^{\prime} D^{\prime}}$ as $\phi$, the cosine rule applied to triangles $O^{\prime} B^{\prime} Q$ and $O^{\prime} B^{\prime} Q^{\prime}$ yielding

$$
\begin{aligned}
& \left|\overline{O^{\prime} Q}\right|^{2}=\left|\overline{O^{\prime} B^{\prime}}\right|^{2}+\left|\overline{O^{\prime} D^{\prime}}\right|^{2}-2\left|\overline{O^{\prime} B^{\prime}}\right|\left|\overline{O^{\prime} D^{\prime}}\right| \sin \phi, \\
& \left|\overline{O^{\prime} Q^{\prime}}\right|^{2}=\left|\overline{O^{\prime} B^{\prime}}\right|^{2}+\left|\overline{O^{\prime} D^{\prime}}\right|^{2}+2\left|\overline{O^{\prime} B^{\prime}}\right| \overline{O^{\prime} D^{\prime}} \mid \sin \phi,
\end{aligned}
$$

where $\left|\overline{O^{\prime} B^{\prime}}\right|$ denotes the length of $\overline{O^{\prime} B^{\prime}}$. Inserting these into Eq. (7), we obtain 


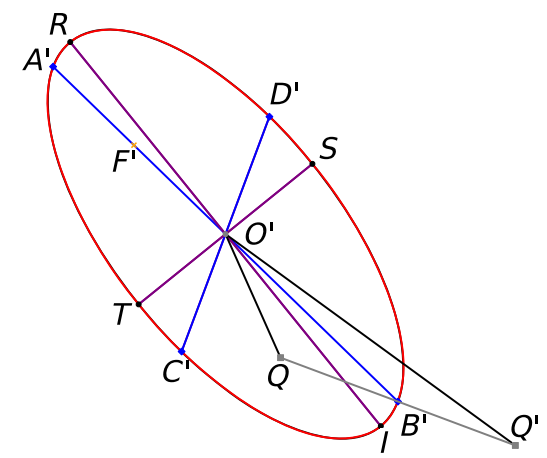

Fig. 5 The projected ellipse (red) has semimajor axis $\overline{I R}$ and semiminor axis $\overline{T S}$ (purple). Both are calculable from the conjugate diameters that are the projections of the semimajor and semiminor axes of the original 3D ellipse $\left(\overline{A^{\prime} B^{\prime}}, \overline{C^{\prime} D^{\prime}}\right.$, blue). The line $\overline{Q Q^{\prime}}$ is drawn such that it is perpendicular to the smaller conjugate diameter $\left(\overline{C^{\prime} D^{\prime}}\right)$ and is bisected by $B^{\prime}:\left|\overline{B^{\prime} Q}\right|=\left|\overline{B^{\prime} Q^{\prime}}\right|=$ $\left|\overline{O^{\prime} C^{\prime}}\right|$. The semimajor axis of the projected ellipse is the angular bisector of $\overline{O^{\prime} Q}$ and $\overline{O^{\prime} Q^{\prime}} .^{15}$ Finally, the semiminor axis $\overline{T S}$ of the projected ellipse is perpendicular to the semimajor axis.

$$
\begin{gathered}
\overline{O^{\prime} R} \cdot \overline{O^{\prime} S}=\left|\overline{O^{\prime} B^{\prime}}\right|\left|\overline{O^{\prime} D^{\prime}}\right| \sin \phi, \\
\left|\overline{O^{\prime} R}\right|^{2}+\left|\overline{O^{\prime} S}\right|^{2}=\left|\overline{O^{\prime} B^{\prime}}\right|^{2}+\left|\overline{O^{\prime} D^{\prime}}\right|^{2} .
\end{gathered}
$$

$|\overline{I R}|$ must be twice the semimajor axis of the projected ellipse $\left(a_{p}\right)$, so

$$
a_{p}=\frac{|\overline{I R}|}{2}=\left|\overline{O^{\prime} R}\right|
$$

and $|\overline{T S}|$ must be twice the semiminor axis of the projected ellipse, so

$$
b_{p}=\frac{|\overline{T S}|}{2}=\left|\overline{O^{\prime} S}\right|
$$

The angle of the semimajor axis of the projected ellipse from $\underline{\hat{x}}$ is calculated using the average of the angles between $\overline{O^{\prime} Q}$ and $\underline{\hat{x}}$ and $\overline{O^{\prime} Q^{\prime}}$ and $\underline{\hat{x}}$ :

$$
\theta=\frac{1}{2}\left(\tan ^{-1}\left(\frac{\overline{O^{\prime} Q} \cdot \hat{\underline{y}}}{\overline{O^{\prime} Q} \cdot \underline{\hat{x}}}\right)+\tan ^{-1}\left(\overline{\overline{O^{\prime} Q^{\prime}} \cdot \hat{y}} \overline{\overline{O^{\prime} Q^{\prime}} \cdot \underline{\hat{x}}}\right)\right) .
$$

Appendix E provides the full expressions for $a_{p}, b_{p}$, and $\theta$ via full expansions of Eqs. (13)-(15), respectively.

Now that we know all of the parameters necessary to describe the projected ellipse, we can standardize this ellipse into a simpler form to simplify subsequent calculations. We define a new frame ( $d r$ as in Fig. 4) as the derotation and geometric centering of the projected orbit such that the semimajor axis of the projected ellipse $\left(\overline{O^{\prime} I}\right)$ is aligned with $\underline{\hat{x}}_{d r}$, the semiminor axis of the projected ellipse $\left(\overline{O^{\prime} S}\right)$ is aligned with $\hat{y}_{d r}$, and $O^{\prime}$ is the origin of the $d r$ coordinate system. The $d r$ coordinates of the star location $F\left(x_{*}, y_{*}\right)$ are given by a simple rotation of the projection of $\overline{O^{\prime} F}$ onto the $\underline{x}, \underline{y}$ plane by angle $\theta$ :

$$
\left[\begin{array}{l}
x_{*} \\
y_{*}
\end{array}\right]_{\mathrm{dr}}=-\left[\begin{array}{cc}
\cos (\theta) & \sin (\theta) \\
-\sin (\theta) & \cos (\theta)
\end{array}\right]\left[\overline{\frac{O^{\prime} F}{O^{\prime} F} \cdot \hat{\hat{x}}}\right]_{X Y Z} .
$$

\subsubsection{Global and local extrema of planet-star separation}

Before solving for the true anomalies where the orbit's projected separation $s$ is equal to $s_{\mathrm{WA}}$ (general working angle separation $\mathrm{WA} d_{i}$ ), we first need to know how many of these $s_{\mathrm{WA}}$-orbit 
intersections we are looking for. We find the expected number of solutions by finding the $s$ extrema throughout the orbit. We do so by solving for the roots of the derivative of the projected planet-star separation.

We start with the general equation for an ellipse:

$$
\left(\frac{x_{e}}{a_{p}}\right)^{2}+\left(\frac{y_{e}}{b_{p}}\right)^{2}=1
$$

where $x_{e}$ and $y_{e}$ are the coordinates of any point on the ellipse and $a_{p}, b_{p}$ are the semimajor and semiminor axes, respectively. We rewrite this in terms of $x_{e}$, giving

$$
y_{e}=b_{p} \sqrt{1-\frac{x_{e}^{2}}{a_{p}^{2}}} .
$$

The projected separation is given by

$$
s^{2}=\left(-x_{*}+x_{e}\right)^{2}+\left(-y_{*}+y_{e}\right)^{2} .
$$

Taking the derivative of Eq. (19) with respect to $x_{e}$, substituting Eq. (18) (and its derivative), and setting it equal to zero, we have

$$
0=\frac{\delta s^{2}}{\delta x_{e}}=-2 x_{*}+2 x_{e}+\frac{2 b_{p} x_{e} y_{*}}{a_{p} \sqrt{a_{p}^{2}-x_{e}^{2}}}-\frac{2 b_{p}^{2} x_{e}}{a_{p}^{2}} .
$$

Isolating the square root term to one side and squaring both sides of the equation gives

$$
\left(-2 x_{*}+2 x_{e}-\frac{2 b_{p}^{2} x_{e}}{a_{p}^{2}}\right)^{2}=\left(\frac{2 b_{p} x_{e} y_{*}}{a_{p} \sqrt{a_{p}^{2}-x_{e}^{2}}}\right)^{2},
$$

which is expanded with coefficients of $x_{e}$, to get the polynomial expression

$$
\begin{aligned}
0= & x_{e}^{4}+\frac{-8 a_{p}^{2} x_{*}+8 b_{p}^{2} x_{*}}{\left(4 a_{p}^{4}-8 a_{p}^{2} b_{p}^{2}+4 b_{p}^{4}\right) / a_{p}^{2}} x_{e}^{3}+\frac{-4 a_{p}^{4}+8 a_{p}^{2} b_{p}^{2}+4 a_{p}^{2} x_{*}^{2}-4 b_{p}^{4}+4 b^{2} y_{*}^{2}}{\left(4 a_{p}^{4}-8 a_{p}^{2} b_{p}^{2}+4 b_{p}^{4}\right) / a_{p}^{2}} x_{e}^{2} \\
& +\frac{8 a_{p}^{4} x_{*}-8 a_{p}^{2} b_{p}^{2} x_{*}}{\left(4 a_{p}^{4}-8 a_{p}^{2} b_{p}^{2}+4 b_{p}^{4}\right) / a_{p}^{2}} x_{e}+\frac{-4 a_{p}^{4} x_{*}^{2}}{\left(4 a_{p}^{4}-8 a_{p}^{2} b_{p}^{2}+4 b_{p}^{4}\right) / a_{p}^{2}} .
\end{aligned}
$$

This expression is a fourth-order polynomial, which we write in standard quartic form as

$$
0=x_{e}^{4}+A_{0} x_{e}^{3}+B_{0} x_{e}^{2}+C_{0} x_{e}+D_{0},
$$

where $A_{0}, B_{0}, C_{0}$, and $D_{0}$ are the constants and functions of $a_{p}, b_{p}, x_{*}$, and $y_{*}$, defined in Appendix F. We now apply the analytical solutions of the general quartic expression in standard form given in Appendix K.

Solving this quartic gives us a set of four $x_{e}$ solutions $\left(\mathbf{x}_{e}\right)$ corresponding to two global extrema and two local extrema (if the latter exist for a particular orbit). Figure 6 shows a schematic representation of an orbit with four extrema. We use the imaginary components of the solutions, geometry of the ellipse, and magnitude of the higher order terms in the quartic solutions to identify which solutions belong to which extrema. We first use the magnitude of the imaginary components of solutions to determine how many extrema there are and filter out solutions that are not extrema. The algebraic solutions of the quartic polynomial all give values in the first quadrant (quadrants 1 to 4 are numbered counter-clockwise such that quadrant 1 has strictly positive coordinates, see the four quadrants of Fig. 6), so we must use geometry of the problem to determine the proper sign of each extremum's true coordinates $\mathbf{x}_{e, g}$ and $\mathbf{y}_{e, g}$ [ $g$ references solutions 0 to 3 Eq. (91)-Eq. (94)]. 


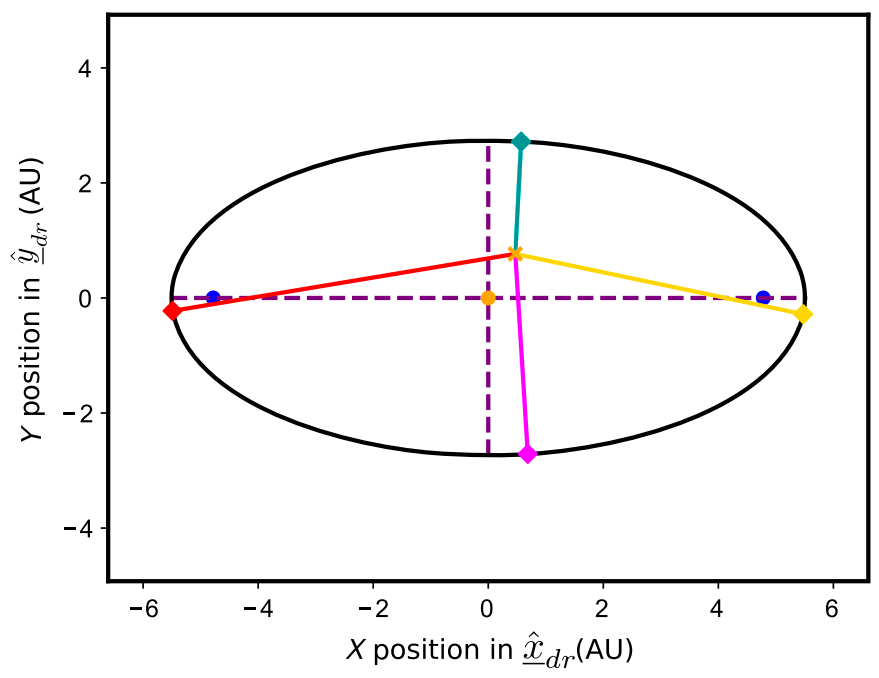

Fig. 6 Planet orbit (black) in the $d r$ frame and planet-star separation extrema. The minimum separation (cyan) always occurs in the same quadrant as the star in the $d r$ frame (orange $\times$ ). The maximum separation (red) always occurs in the quadrants opposite the star. The local minimum separation (magenta) and local maximum separation (gold) occur in the same half-plane about the $y$ axis as the star, but in opposite half-planes about the $x$ axis as the star (fourth quadrant). This configuration applies to the vast majority of orbits. A small number of edge cases exist, including circular orbits and some edge on orbits. The blue dots represent the foci of the projected ellipse in the $d r$ frame, the orange dot is the ellipse center, and the purple dashed lines are the semimajor and semiminor axes.

All numerical solutions to the quartic have some degree of imaginary component due to accumulation of numerical errors. Quartic solution sets with only two extrema will have two real solutions (solutions with small imaginary components only due to numerical error) and two solutions with large imaginary components (algebraic solutions that are artifacts to be thrown away). The majority of KOE have only two $s$ extrema. The only case in which no extrema exist is in a circular face-on orbit. In this specific case, all solutions to the quartic will be nearly identical and have large imaginary components, and the resulting $s$ extrema will be identical $\left(s_{\min }=s_{\max }\right)$. We assume that all solutions are real if $\left|\mathfrak{s}\left(\mathbf{x}_{e, g}\right)\right|<10^{-5} \forall g \in\{0,1,2,3\}$. We assume only two solutions are real if $\left|\mathfrak{I}\left(\mathbf{x}_{e}\right)\right|<10^{-5}$ for only two solutions. We define a new ordered set containing either two or four elements depending on magnitude of the imaginary components as

$$
\mathbf{x}_{\mathfrak{R}}=\left\{\left|\Re\left(\mathbf{x}_{e, g}\right)\right|:\left|\mathfrak{I}\left(\mathbf{x}_{e, g}\right)\right|<10^{-5} \forall g \in 0 . .3\right\},
$$

where the absolute value is due to the algebraic solutions of the quartic being only defined in the first quadrant.

We now know the number of expected solutions from the dimension of $\mathbf{x}_{\mathfrak{R}}$ but need to leverage the quartic algebraic solution and geometry to determine which components belong to which quadrant. For orbits with $\mathbf{x}_{\mathfrak{R}}$ containing only two solutions, the first two solutions to the quartic ( $\mathbf{x}_{\mathfrak{R}, 0}$ and $\mathbf{x}_{\mathfrak{R}, 1}$ ) produce the largest and smallest $\mathbf{x}_{\mathfrak{R}, g}-x_{*}$, respectively. Due to the shape of an ellipse, these must necessarily produce $s_{\min }$ and $s_{\max }$. We define the set $\mathbf{y}_{\Re}$ as the application of Eq. (18) to each element of $\mathbf{x}_{\mathfrak{R}}$. We define four separation quantities from the possible sign combinations of the coordinate magnitudes in $\mathbf{x}_{\Re}$ and $\mathbf{y}_{\Re}$ as

$$
\begin{aligned}
& s_{ - \pm 0}=\sqrt{\left(\mathbf{x}_{\Re, 0}-x_{*}\right)^{2}+\left(\mathbf{y}_{\Re, 0} \pm y_{*}\right)^{2}}, \\
& s_{ + \pm 0}=\sqrt{\left(\mathbf{x}_{\Re, 0}+x_{*}\right)^{2}+\left(\mathbf{y}_{\Re, 0} \pm y_{*}\right)^{2}}
\end{aligned}
$$




$$
\begin{aligned}
& s_{ - \pm 1}=\sqrt{\left(\mathbf{x}_{\Re, 1}-x_{*}\right)^{2}+\left(\mathbf{y}_{\Re, 1} \pm y_{*}\right)^{2}}, \\
& s_{ + \pm 1}=\sqrt{\left(\mathbf{x}_{\Re, 1}+x_{*}\right)^{2}+\left(\mathbf{y}_{\Re, 1} \pm y_{*}\right)^{2}} .
\end{aligned}
$$

Typically, $s_{--1}$ is smaller than $s_{--0}$, but in $\ll 0.01 \%$ of cases, $s_{--0}$ can be the smallest separation due to numerical error. (In this case, we ensure $\left|s_{--1}-s_{--0}\right|<10^{-8}$ and swap values.) In addition to $s_{--1}<s_{--0}$, we also know that $s_{-+0}<s_{-+1}, s_{+-1}<s_{+-0}$, and $s_{++1}<s_{++0}$ for all cases. Using this knowledge, we reduce the number of comparisons that we need to find the minimum planet-star separation to

$$
s_{\min }=\left\{\begin{array}{l}
s_{--1} \text { where }\left(s_{--1}<s_{-+0}\right) \text { and }\left(s_{--1}<s_{+-1}\right) \text { and }\left(s_{--1}<s_{++1}\right) \\
s_{-+0} \text { where }\left(s_{-+0}<s_{--1}\right) \text { and }\left(s_{-+0}<s_{+-1}\right) \text { and }\left(s_{-+0}<s_{++1}\right) \\
s_{+-1} \text { where }\left(s_{+-1}<s_{-+0}\right) \text { and }\left(s_{+-1}<s_{--1}\right) \text { and }\left(s_{+-1}<s_{++1}\right) \\
s_{++1} \text { where }\left(s_{++1}<s_{-+0}\right) \text { and }\left(s_{++1}<s_{+-1}\right) \text { and }\left(s_{++1}<s_{--1}\right)
\end{array}\right.
$$

The maximum separation similarly is found as

$$
s_{\max }=\left\{\begin{array}{l}
s_{--0}, \text { where }\left(s_{--0}>s_{-+0}\right) \text { and }\left(s_{--0}>s_{+-1}\right) \text { and }\left(s_{--0}>s_{++0}\right), \\
s_{-+0}, \text { where }\left(s_{-+0}>s_{--0}\right) \text { and }\left(s_{-+0}>s_{+-1}\right) \text { and }\left(s_{-+0}>s_{++0}\right) \\
s_{+-1}, \text { where }\left(s_{+-1}>s_{-+0}\right) \text { and }\left(s_{+-1}>s_{--0}\right) \text { and }\left(s_{+-1}>s_{++0}\right) \\
s_{++0}, \text { where }\left(s_{++0}>s_{-+0}\right) \text { and }\left(s_{++0}>s_{+-1}\right) \text { and }\left(s_{++0}>s_{--0}\right) .
\end{array}\right.
$$

We are able to find $x_{e}$ and $y_{e}$ of the minimum and maximum separation diamonds drawn in Fig. 6 using the same logic as for finding $s_{\min }$ and $s_{\max }$.

For the orbits with four solutions in $\mathbf{x}_{\mathfrak{R}}$, the first two elements will always be the global extrema, and the last two elements will be the local extrema, which are given by

$$
\begin{gathered}
s_{\min }=s_{--1}, \\
s_{\max }=s_{++0}, \\
s_{\text {lmin }}=\left\{\begin{array}{ll}
s_{-+3}, & s_{-+2}>s_{-+3} \\
s_{-+2}, & \text { else }
\end{array},\right. \\
s_{\operatorname{lmax}}= \begin{cases}s_{-+2}, & s_{-+2}>s_{-+3} \\
s_{-+3}, & \text { else }\end{cases}
\end{gathered}
$$

where $s_{-+2}$ and $s_{-+3}$ are calculated in the same manner as $s_{-+0}$ and $s_{-+0}$. We are able to find the coordinates of all four using the same logic as for finding $s_{\min }, s_{\max }, s_{\text {lmin }}$, and $s_{\text {lmax }}$. This procedure yields the coordinates of all existing extrema in the $d r$ frame. To find their locations on the projection of orbit in the plane of the sky, we apply the inverse of Eq. (16).

\subsubsection{Intersections between a circle and an ellipse}

We find $s_{\mathrm{WA}}$-orbit intersections by formulating the circle-ellipse intersections as another quartic, solving this, and assigning the algebraic solutions to intersections. We assign solutions using the number of $s$ extrema, the size of the $s_{\mathrm{WA}}$ intersecting circle relative to these $s$ extrema, and the ellipse geometry. To formulate the $s_{\mathrm{WA}}$-orbit intersections as a quartic, we start with Eq. (19) and substitute in Eq. (18). This gives us a separation equation solely as a function of $x_{e}$ and star location. We expand and transform this into a general polynomial of $x_{e}$ with a general $s$ 


$$
\begin{aligned}
0= & \left(\frac{a_{p}^{4}-2 a_{p}^{2} b_{p}^{2}+b_{p}^{4}}{a_{p}^{4}}\right) x_{e}^{4}+\left(\frac{-4 a_{p}^{2} x+4 b_{p}^{2} x_{*}}{a_{p}^{2}}\right) x_{e}^{3} \\
& +\left(\frac{2 a_{p}^{2} b_{p}^{2}-2 a_{p}^{2} s^{2}+6 a_{p}^{2} x_{*}^{2}+2 a_{p}^{2} y_{*}^{2}-2 b_{p}^{4}+2 b_{p}^{2} s^{2}-2 b_{p}^{2} x_{*}^{2}+2 b_{p}^{2} y_{*}^{2}}{a_{p}^{2}}\right) x_{e}^{2} \\
& +\left(-4 b_{p}^{2} x+4 s^{2} x-4 x_{*}^{3}-4 x_{*} y_{*}^{2}\right) x_{e} \\
& +\left(b_{p}^{4}-2 b_{p}^{2} s^{2}+2 b_{p}^{2} x_{*}^{2}-2 b_{p}^{2} y_{*}^{2}+s^{4}-2 s^{2} x_{*}^{2}-2 s^{2} y_{*}^{2}+x_{*}^{4}+2 x_{*}^{2} y_{*}^{2}+y_{*}^{4}\right) .
\end{aligned}
$$

As in Sec. 2.1.2, we divide by the leading coefficient to convert this to the general quartic form:

$$
0=x_{e}^{4}+A_{1} x_{e}^{3}+B_{1} x_{e}^{2}+C_{1} x_{e}+D_{1},
$$

with $A_{1}, B_{1}, C_{1}$, and $D_{1}$ given in Appendix G. We solve this using the general quartic solution as given in Appendix K. This results in a solution for the $x_{e}$ of intersections in the first quadrant of the $d r$ frame.

We always have four algebraic $x_{e}$ solutions that may or may not correspond to actual $s_{\mathrm{WA}^{-}}$ orbit intersections. The number of $s$ extrema (either two or four) and projected separation relative to these extrema determine how many intersections will occur. (If we expect two intersections, then two of the four algebraic solutions must be real solutions, and the other two are some combination of repeated roots or non-physical imaginary solutions.) In each of these cases, we must handle the assignment of $x_{e}$ and $y_{e}$ solutions to the correct quadrants. Figure 7 shows a schematic representation of two cases corresponding to four total intersections.

For KOE with four extrema and $s_{\min }<s_{\mathrm{WA}}<s_{\operatorname{lmin}}$, we know that there will be two intersections on the same $y$ side of the ellipse as the star (quadrants 1 or 2 in the $d r$ frame). Of the four quartic solutions to Eq. (36) that we have to choose from, we know that $x_{0}$ is one of them. The other solution could either be $x_{1}$ if $\mathfrak{I}\left(x_{1}\right)<10^{-9}$ or $x_{3}$ if $\mathfrak{I}\left(x_{1}\right)>10^{-9}$.

For KOE with four extrema and $s_{\mathrm{Imax}}<s_{\mathrm{WA}}<s_{\max }$, we know that there will be two intersections on the opposite $x$ side of the ellipse as the star (quadrants 2 and 3 in the $d r$ frame). In all cases, $x_{0}$ and $x_{1}$ are the intersection solutions. This is because the first two solutions have the largest term in the quartic solution [Eqs. (91) and (92)]. $x_{1}$ is slightly smaller than $x_{0}$ because it subtracts the second largest term. The relative magnitudes of $x_{0}$ and $x_{1}$ determine that $y_{0}$ occurs in the same side of the ellipse as the star and $y_{1}$ must occur on the opposite side of the ellipse as the star. Therefore, $\left(x_{0}, y_{0}\right)$ occurs in quadrant 2 , and $\left(x_{1}, y_{1}\right)$ occurs in quadrant 3 .

For KOE with four extrema and $s_{\mathrm{lmin}}<s_{\mathrm{WA}}<s_{\mathrm{lmax}}$, we know that there will be four intersections. Unlike in Sec. 2.1.2 and the rest of this paper where $x_{0}$ through $x_{3}$ are ordered as in Appendix $\mathrm{K}$, we order $x_{h}$ based off $\Delta x_{h}=\left|x_{h}-x_{*}\right|$. We order the quartic solutions from $x_{0}$ to $x_{3}$ such that $x_{0}$ is where $\min \left(\left\{\Delta x_{h} \forall h \in\{0,1,2,3\}\right\}\right)$ and $x_{3}$ is where $\max \left(\left\{\Delta x_{h} \forall h \in\right.\right.$ $\{0,1,2,3\}\})$. These newly ordered $\Delta x_{h}$ correspond to those shown in Fig. 7. The $\left(x_{2}, y_{2}\right)$ intersection occurs in either quadrant 1 or 2 , but it is always above and to the left of the star in the $d r$ frame and has the second largest $\Delta x_{h}$ component. The $\left(x_{3}, y_{3}\right)$ intersection occurs in either quadrant 1 or 4 , but it always has the largest $\Delta x_{h}$ component. We resolve the sign of $y_{3}$ by testing it in both quadrants. In $>99.992 \%$ of cases, we assign $y_{3}$ to quadrant 1 as in Fig. 7(a), but for a minority of cases, its correct assignment is quadrant 4 as in Fig. 7(b). The $\left(x_{1}, y_{1}\right)$ intersection always occurs in quadrant 4 . The $\left(x_{0}, y_{0}\right)$ intersection occurs in either quadrant 3 or quadrant 4 , but it is always below and to the left of the star in the $d r$ frame and has the smallest $\Delta x_{h}$ component.

For KOE with two extrema and $s_{\min }<s_{\mathrm{WA}}<s_{\max }$, we know that there will be two circleellipse intersections. The KOE determine where the star is located in the $d r$ frame. The location of the star in the $d r$ frame relative to the vertices of the projected ellipse $\left[\left(0, b_{p}\right),\left(0,-b_{p}\right)\right.$, $\left(-a_{p}, 0\right)$, and $\left.\left(a_{p}, 0\right)\right]$ determines the star-vertex separation ordering and subsequently to which quadrants the two intersection solutions belong. Instead of calculating the star-vertex separation for each orbit, we divide the first quadrant into four regions that specify the star location type [types 0 to 3 as indicated in Fig. 8(a)]. This means that any KOE with the star in location type 2 has the associated star-vertex separation ordering.

Using the equidistant lines between ellipse vertices, we divide the first quadrant into four regions (types 0 to 3 ) as shown in Fig. 8(a). Regions 0 and 2 are divided by the line defined by 

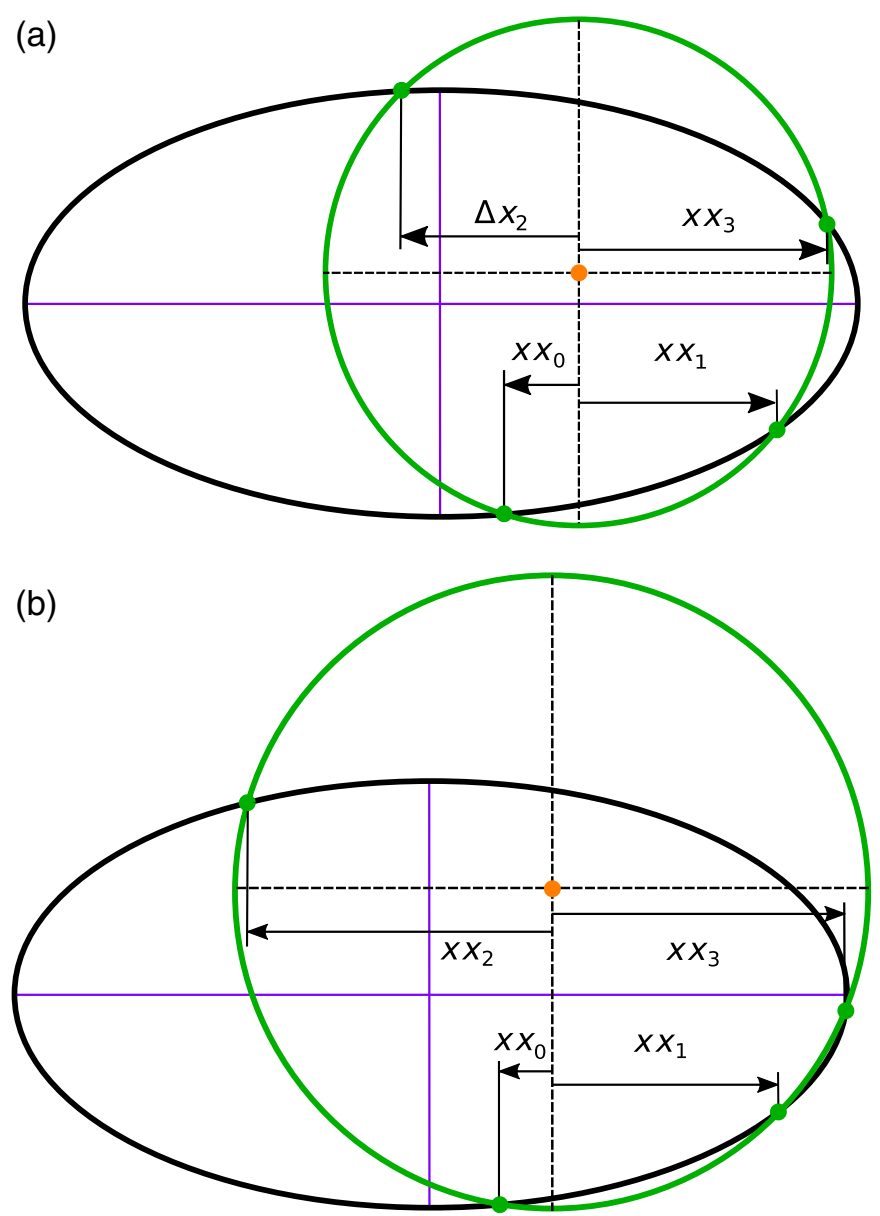

Fig. 7 Diagrams of orbits where the projected ellipse in the $d r$ frame produces four intersections (green dots) with the $s_{\text {WA }}$ separation circle (green circle). The general orbit's projected ellipse (black) is centered at the origin, and the projected ellipse axes (purple) define the $\overline{\hat{x}}_{d r}$ and $\overline{\hat{y}}_{d r}$ axes. (Note that the projected ellipse semimajor axis has been derotated such that the star is always located in the first quadrant.) The separation circle center (orange dot) is the star's location relative to the orbit's projected ellipse. The $x_{h}$ points are the quartic solutions, where subscripts are reordered in ascending distance from the star's $x$ position. This ordering means that $x_{2}$ must always occur in the fourth quadrant, $x_{0}$ may occur in either the first or second quadrants, and $x_{1}$ may occur in either the third or fourth quadrants. For $99.992 \%$ of KOE sampled from the SAG13 population that produce four intersections, the $x_{3}$ intersection occurs in the first quadrant (a). The other $0.008 \%$ of KOE result in the $x_{3}$ intersection occurring in the fourth quadrant (b).

$$
y_{a_{p}-x, b_{p}-y}=\frac{a_{p}}{b_{p}} x+\frac{a_{p}^{2}}{2 b_{p}}-\frac{b_{p}}{2},
$$

where a projected ellipse and star is of type 0 if $y_{*}>y_{a_{p}-x, b_{p}-y}\left(x_{*}\right)$ and one of types 1, 2, or 3 otherwise depending on the other equidistant lines. Similarly, regions 1 and 2 are divided by the line defined by

$$
y_{a_{p}+x, b_{p}-y}=-\frac{a_{p}}{b_{p}} x+\frac{a_{p}^{2}}{2 b_{p}}-\frac{b_{p}}{2},
$$

regions 2 and 3 are divided by the line defined by

$$
y_{a_{p}+x, b_{p}+y}=\frac{a_{p}}{b_{p}} x-\frac{a_{p}^{2}}{2 b_{p}}+\frac{b_{p}}{2},
$$

and the star-ellipse classification type is calculated similar to type 0 . 
(a) Ellipse extrema distance ordering by star location

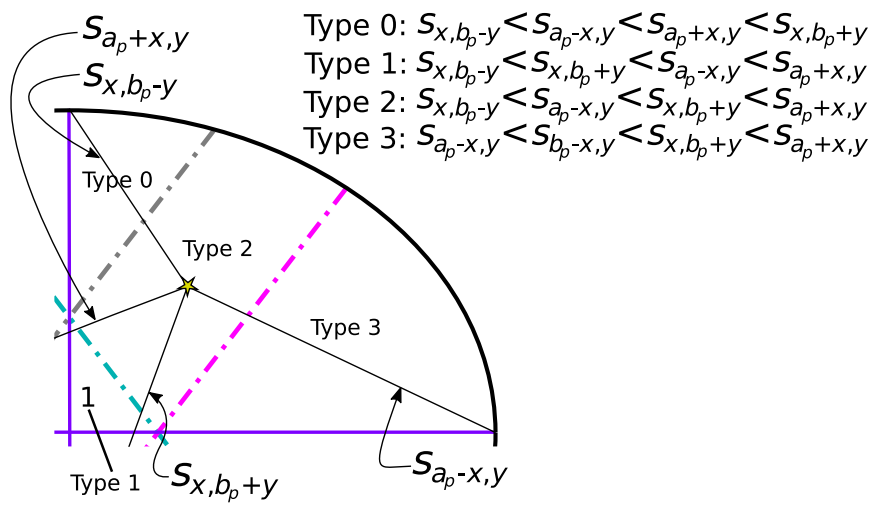

(b) closest vertex

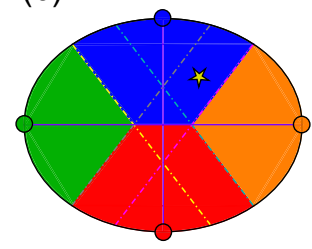

(d) $3^{\text {rd }}$ closest vertex

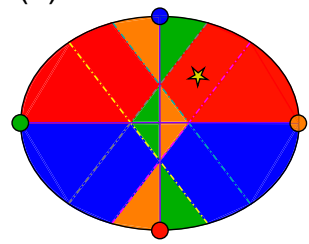

(c) $2^{\text {nd }}$ closest vertex

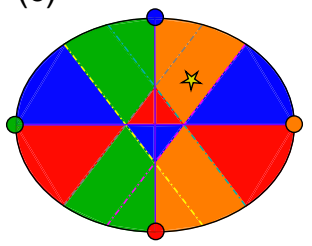

(e) $4^{\text {th }}$ closest vertex

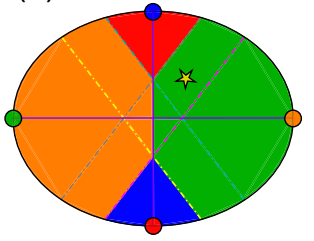

Fig. 8 The regions identifying the star type and which vertices are closest to the host star. (a) The first quadrant of a projected ellipse (black curve) with the semimajor and semiminor axes (purple) and three dashed lines dividing the quadrant into four regions defining the separation ordering. The pink dashed line represents the line of points equidistant from $\left(0, b_{p}\right)$ and $\left(a_{p}, 0\right)$. The gray dashed line represents the line of points equidistant from $\left(-a_{p}, 0\right)$ and $\left(0,-b_{p}\right)$. The turquoise dashed line represents the line of points equidistant from $\left(0,-b_{p}\right)$ and $\left(a_{p}, 0\right)$. The yellow dashed line represents the line of points equidistant from $\left(-a_{p}, 0\right)$ and $\left(0, b_{p}\right)$. We say that the star in $(a)$ is a type 2 star and has the associated separation ordering. (b)-(e) Color each region of the ellipse, identifying which vertex is closest, second closest, third closest, and fourth closest. The star in (a) is type 2 and has the top vertex as the closest as seen in (b). These plots are based off the projected ellipse of a planet with $a=0.40 \mathrm{AU}, e=0.23, i=0.69 \mathrm{rad}, \Omega=3.49 \mathrm{rad}$, and $\omega=5.64 \mathrm{rad}$.

An example star is shown in Fig. 8(a). This star location is type 2 because it is in the region bounded by the three dashed lines (gray, pink, and teal). This type 2 star has the separation ordering indicated in Fig. 8(a) and Table 1. Let us consider a $s_{\mathrm{WA}}$ separation circle such that $s_{a_{p}-x_{*}, y_{*}}<s_{\mathrm{WA}}<s_{x_{*}, b_{p}+y_{*}}$. We, therefore, know that the two intersections must occur in quadrant 2 and quadrant 4 of the $d r$ frame.

The distances $s_{x_{*}, b_{p}+y_{*}}, s_{x_{*}, b_{p}-y_{*}}, s_{a_{p}+x_{*}, y_{*}}$, and $s_{a_{p}-x_{*}, y_{*}}$ in Fig. 8(a) are the distances of the star to each of the ellipse vertices and are calculated by

$$
\begin{aligned}
& s_{x_{*}, b_{p} \pm y_{*}}=\sqrt{x_{*}^{2}+\left(b_{p} \pm y_{*}\right)^{2}}, \\
& s_{a_{p} \pm x_{*}, y_{*}}=\sqrt{\left(a_{p} \pm x_{*}\right)^{2}+y_{*}^{2}} .
\end{aligned}
$$

Figure 8(a) and Table 1 define orbit types sorted by vertex distances from smallest to largest based on star location type in the first quadrant of the $d r$ frame. 
Table 1 Separation order from smallest to largest by star location type.

\begin{tabular}{|c|c|c|c|c|c|}
\hline \multirow[b]{2}{*}{ Type } & \multirow[b]{2}{*}{ Condition } & \multicolumn{4}{|c|}{ Separation order from smallest to largest } \\
\hline & & First & Second & Third & Fourth \\
\hline 0 & $s_{a_{p}+x_{*}, y_{*}}<s_{x_{*}, b_{p}+y_{*}}$ & $s_{x_{*}, b_{p}-y_{*}}$ & $s_{a_{p}-x_{*}, y_{*}}$ & $s_{a_{p}+x_{*}, b_{p}}$ & $s_{x_{*}, b_{p}+y_{*}}$ \\
\hline 1 & $s_{x_{*}, b_{p}+y_{*}}<s_{a_{p}-x_{*}, y_{*}}$ & $s_{X_{*}, b_{p}-y_{*}}$ & $s_{X_{*}, b_{p}+y_{*}}$ & $s_{a_{p}-x_{*}, y_{*}}$ & $s_{a_{\rho}+x_{*}, y_{*}}$ \\
\hline 2 & $\begin{array}{l}s_{a_{p}-X_{*}, y_{*}}<s_{X_{*}, b_{p}+y_{*}} \\
s_{X_{*}, b_{p}+y_{*}}<s_{a_{p}+X_{*}, y_{*}} \\
s_{X_{*}, b_{p}-y_{*}}<s_{a_{p}-X_{*}, y_{*}}\end{array}$ & $s_{x_{*}, b_{p}-y_{*}}$ & $s_{a_{p}-x_{*}, y_{*}}$ & $s_{X_{*}, b_{p}+y_{*}}$ & $s_{a_{p}+x_{*}, y_{*}}$ \\
\hline 3 & $s_{a_{p}-X_{*}, y_{*}}<s_{X_{*}, b_{p}-y_{*}}$ & $s_{a_{p}-X_{*}, y_{*}}$ & $s_{X_{*}, b_{p}-y_{*}}$ & $s_{X_{*}, b_{p}+y_{*}}$ & $s_{a_{p}+x_{*}, y_{*}}$ \\
\hline
\end{tabular}

Finally, after determining the correct number of intersection solutions and the proper quadrants to which these solutions belong, we rerotate and translate the intersection solution locations back into the $2 \mathrm{D}$ projection of the $3 \mathrm{D}$ orbit.

\section{2 $\Delta$ mag Intersections}

In this section, we present our method for calculating the values of $\nu$ on a planet's orbit where the planet has a specific value of $\Delta \mathrm{mag}_{\text {lim }}$, called $\Delta$ mag intersections. As in Sec. 2.1, to compute these solutions, we first need to calculate all $\Delta$ mag extrema $\left(\Delta \operatorname{mag}_{\min }, \Delta \operatorname{mag}_{\max }, \Delta \operatorname{mag}_{\operatorname{lmin}}\right.$, and $\left.\Delta \operatorname{mag}_{\operatorname{lmax}}\right)$. The process for calculating $\Delta$ mag extrema is detailed in Appendix $\mathrm{C}$, but briefly included as follows:

1. express $\Delta$ mag as a polynomial in $\cos (\nu)$;

2. find the values of $\nu$ and $\Delta$ mag for all polynomial roots; and

3. remove invalid and duplicate solutions.

The general process for calculating the true anomalies where a planet has $\Delta \operatorname{mag}_{\text {lim }}$ is similar to the extrema-finding process but with some minor modifications. After calculating the $\Delta$ mag extrema, we determine how many $\Delta$ mag intersections a given orbit should have with a particular $\Delta \operatorname{mag}_{\text {lim }}$ value. If $\Delta \operatorname{mag}_{\text {lim }}<\Delta \operatorname{mag}_{\min }$ or $\Delta \operatorname{mag}_{\text {lim }}>\Delta \operatorname{mag}_{\max }$, there are no intersections. If $\Delta \operatorname{mag}_{\min }<\Delta \operatorname{mag}_{\text {lim }}<\Delta \operatorname{mag}_{\text {Imin }}$ or $\Delta \operatorname{mag}_{\operatorname{lmax}}<\Delta \operatorname{mag}_{\text {lim }}<\Delta \operatorname{mag}_{\max }$, then there are exactly two intersections. If $\Delta \operatorname{mag}_{\operatorname{lmin}}<\Delta \operatorname{mag}_{\lim }<\Delta \operatorname{mag}_{\operatorname{lmax}}$, then there are exactly four intersections. When the orbit does not contain local $\Delta$ mag extrema, there are just two $\Delta$ mag intersections if $\Delta \operatorname{mag}_{\min }<\Delta \operatorname{mag}_{\text {lim }}<\Delta \operatorname{mag}_{\max }$.

Knowing the number of solutions to expect, we follow the same steps as above: finding a governing polynomial equation, solving for its roots (represented by the ordered set $\mathbf{x}$ ), and filtering out the relevant solutions. We additionally throw out any solutions with large errors from the input $\Delta \mathrm{mag}_{\text {lim }}$. The full process outline is included in Appendix D and discussed in depth below.

We start with the definition of $\Delta$ mag given in Eq. (1). Although this expression contains multiple terms that are fully defined by an orbit's KOE, it is also a function of other planet properties, including the planet's radius, geometric albedo, and phase function. To make the mathematical development presented below tractable, we assume that the quasi-Lambert phase function ${ }^{17}$ is a sufficient approximation of any planet's phase function. In general, the quasiLambert phase function is a better representation of the Earth's phase function than the Lambert phase function.

This quasi-Lambert phase function is given by

$$
\Phi_{L}(\beta)=\cos ^{4}\left(\frac{\beta}{2}\right)
$$

We take advantage of this function's form by substituting the half-angle formula: 


$$
\cos \left(\frac{\beta}{2}\right)=\sqrt{\frac{1+\cos (\beta)}{2}}
$$

At the same time, from the orbital geometry defined in Fig. 3 and Eq. (3), we write

$$
\beta=\cos ^{-1}(\sin (i) \sin (\nu+\omega)),
$$

where the final term is expanded by the angle addition formula as

$$
\sin (\nu+\omega)=\sin (\nu) \cos (\omega)+\cos (\nu) \sin (\omega) .
$$

Substituting in $\beta$ from Eq. (44) expanded with Eq. (45) into Eq. (43) allows us to reduce the order of the fully substituted Eq. (42). Note that this expression for $\beta$ depends on making the approximation that the observer-star and observer-planet vectors are parallel, which introduces minor error given the large distances to even the nearest stars. ${ }^{18}$

After making these substitutions, as well as taking Eq. (4) for the planet-star distance term $\left|\underline{r}_{k / i}\right|$, simplifying, and collecting all of the non-orbital planet parameters on the left side, we find

$$
\frac{a^{2}\left(1-e^{2}\right)^{2}}{p R^{2}} 10^{-2.5 \Delta \mathrm{mag}}=\frac{1}{4}(e \cos (\nu)+1)^{2}(1+\sin (i) \cos (\omega) \sin (\nu)+\sin (i) \sin (\omega) \cos (\nu))^{2} .
$$

We now have an expression that isolates terms containing $\nu$ and can be decomposed into a numerically solvable polynomial. To improve solving efficiency and determine which subset of planets should have $\nu$ solutions for a given $\Delta$ mag, we need to first calculate the $\Delta$ mag extrema over the full orbit. The outline of the process for calculating these $\Delta$ mag extrema is included in Appendix C.

To calculate these $\Delta$ mag extrema, we first find the derivative of Eq. (46) and multiply by two (for simplification purposes) to get

$$
\begin{aligned}
0= & -e^{2} \sin ^{2}(i) \sin ^{3}(\nu) \cos (\nu) \cos ^{2}(\omega)-3 e^{2} \sin ^{2}(i) \sin ^{2}(\nu) \sin (\omega) \cos ^{2}(\nu) \cos (\omega) \\
& -2 e^{2} \sin ^{2}(i) \sin (\nu) \sin ^{2}(\omega) \cos ^{3}(\nu)+e^{2} \sin ^{2}(i) \sin (\nu) \cos ^{3}(\nu) \cos ^{2}(\omega) \\
& +e^{2} \sin ^{2}(i) \sin (\omega) \cos ^{4}(\nu) \cos (\omega)-2 e^{2} \sin (i) \sin ^{2}(\nu) \cos (\nu) \cos (\omega) \\
& -3 e^{2} \sin (i) \sin (\nu) \sin (\omega) \cos ^{2}(\nu)+e^{2} \sin (i) \cos ^{3}(\nu) \cos (\omega)-e^{2} \sin (\nu) \cos (\nu) \\
& -e \sin ^{2}(i) \sin ^{3}(\nu) \cos ^{2}(\omega)-4 e \sin ^{2}(i) \sin ^{2}(\nu) \sin (\omega) \cos (\nu) \cos (\omega) \\
& -3 e \sin ^{2}(i) \sin (\nu) \sin ^{2}(\omega) \cos ^{2}(\nu)+2 e \sin ^{2}(i) \sin (\nu) \cos ^{2}(\nu) \cos ^{2}(\omega) \\
& +2 e \sin ^{2}(i) \sin (\omega) \cos ^{3}(\nu) \cos (\omega)-2 e \sin (i) \sin ^{2}(\nu) \cos (\omega) \\
& -4 e \sin (i) \sin (\nu) \sin (\omega) \cos (\nu)+2 e \sin (i) \cos ^{2}(\nu) \cos (\omega)-e \sin (\nu) \\
& -\sin ^{2}(i) \sin 2(\nu) \sin (\omega) \cos (\omega)-\sin ^{2}(i) \sin (\nu) \sin ^{2}(\omega) \cos (\nu) \\
& +\sin ^{2}(i) \sin (\nu) \cos (\nu) \cos { }^{2}(\omega)+\sin ^{2}(i) \sin (\omega) \cos ^{2}(\nu) \cos (\omega) \\
& -\sin (i) \sin (\nu) \sin (\omega)+\sin (i) \cos (\nu) \cos (\omega),
\end{aligned}
$$

which is a function of the $\sin (\nu)$ and $\cos (\nu)$ terms. We now fully expand this expression and substitute in

$$
\sin (\nu)=\sqrt{1-\cos ^{2}(\nu)}
$$

to get an expression in $\cos (\nu)$ only. We define $x=\cos (\nu)$, isolate the $\sqrt{1-x^{2}}$ term, square both sides, and expand to get an eighth degree polynomial in $x$ of the form

$$
0=A_{2} x^{8}+B_{2} x^{7}+C_{2} x^{6}+D_{2} x^{5}+E_{2} x^{4}+F_{2} x^{3}+G_{2} x^{2}+H_{2} x+I_{2} .
$$

The coefficients of this expression are included in Eq. (75) in Appendix H. 
Although eighth degree polynomials do not have analytical solutions, a numerical root solver can determine the eight roots of this function denoted as the ordered set $\mathbf{x}$. We discard any $\mathbf{x}_{p}>1 \forall p \in\{0 \ldots 8\}, \mathbf{x}_{p}<-1 \forall p \in\{0 \ldots 8\}$, or solutions with large imaginary components. We then calculate the remaining true anomaly solutions by $\nu_{0}=\cos ^{-1}(\mathbf{x})$ and $\nu_{1}=$ $2 \pi-\nu_{0}$. Of the remaining valid solutions, we identify whether each solution is an extremum by evaluating whether $\Delta \operatorname{mag}\left(\boldsymbol{\nu}_{0} \pm \delta \nu\right)$ are both larger or both smaller than $\Delta \operatorname{mag}\left(\boldsymbol{\nu}_{0}\right)$. If identified as a potential extremum, the smallest and largest extrema are assigned to $\Delta \mathrm{mag}_{\min }$ and $\Delta \operatorname{mag}_{\max }$. The remaining extrema are checked for duplicates, which are identified by solutions with $(\nu, \Delta$ mag) values close to existing extrema. If any solutions are remaining, an additional check and assignment is then made for the local extrema. Through this process, a solution identified in $\boldsymbol{\nu}_{0}$ has the associated solution in $\boldsymbol{\nu}_{1}$ removed.

To calculate the $\Delta$ mag intersections, we apply the same process for turning Eq. (46) into a polynomial as in the $\Delta$ mag extrema calculation. The full outline for calculating the $\Delta$ mag intersections is given in Appendix D. By following this process, we arrive at

$$
\xi=A_{3} x^{8}+B_{3} x^{7}+C_{3} x^{6}+D_{3} x^{5}+E_{3} x^{4}+F_{3} x^{3}+G_{3} x^{2}+H_{3} x+I_{3},
$$

where $\xi$ is the collection of constants on the left side of Eq. (46). The coefficients of this polynomial are given in Eq. (76) of Appendix I.

We again use a numerical root solver to find the roots of this function $\mathbf{x}$ and again discard all $\mathbf{x}_{p}>1 \forall p \in\{0 \ldots 8\}, \mathbf{x}_{p}<-1 \forall p \in\{0 \ldots 8\}$, and solutions with large imaginary components. We then calculate the true anomalies of all remaining solutions by $\nu_{0}=\cos ^{-1}(\mathbf{x})$ and $\boldsymbol{\nu}_{1}=2 \pi-\boldsymbol{\nu}_{0}$. We then evaluate $\Delta \operatorname{mag}_{0}=\Delta \operatorname{mag}\left(\boldsymbol{\nu}_{0}\right)$ and $\Delta \operatorname{mag}_{1}=\Delta \operatorname{mag}\left(\boldsymbol{\nu}_{1}\right)$ and remove solutions where $\left|\Delta \operatorname{mag}_{0}-\Delta \operatorname{mag}_{\text {lim }}\right|>0.01$ and $\left|\Delta \operatorname{mag}_{1}-\Delta \operatorname{mag}_{\text {lim }}\right|>0.01$. (Note that 0.01 is $< \pm 0.08 \%$ error on $\Delta$ mag.) We do an iterative process of selecting $(\nu, \Delta$ mag), which are unique (not duplicate solutions) and are closest to the expected $\Delta \operatorname{mag}_{\text {lim }}$, until we have the expected number of solutions. In the majority of cases in which solutions exist, there are generally only two viable solutions, the assignment of which is simple. In some cases, the solution selection is more ambiguous as double roots are possible.

\section{$2.3 \nu$ from $X$ and $Y$}

Sections 2.1.2, 2.1.3, and 2.2 give locations of extrema at intersections in the plane of the sky of the form $(x, y)$, but we need to know the true anomalies of the orbit where these intersections occur.

We start with $X$ and $Y$ in Eq. (3) and solve for $(1+e \cos (\nu)) /\left(a\left(1-e^{2}\right)\right)$, resulting in

$$
\frac{1+e \cos (\nu)}{a\left(1-e^{2}\right)}=\frac{1}{Y}[\sin \Omega \cos (\omega+\nu)+\cos \Omega \sin (\omega+\nu) \cos i]
$$

and

$$
\frac{1+e \cos (\nu)}{a\left(1-e^{2}\right)}=\frac{1}{X}[\cos (\Omega) \cos (\omega+\nu)-\sin (\Omega) \sin (\omega+\nu) \cos (i)] .
$$

In both of these expressions, we substitute angle addition formulas of $\sin (\omega+\nu)$ and $\cos (\omega+\nu)$ and subsequently set the two equations equal to each other.

We set Eqs. (51) and (52) equal to each other, expand, isolate a $\cos (\nu)$ term and $\sin (\nu)$ term, solve for $\nu$, and rearrange to get

$$
\nu=\tan ^{-1}\left[\frac{-\frac{X}{Y} \sin \Omega \cos \omega-\frac{X}{Y} \cos \Omega \cos i \sin \omega+\cos \Omega \cos \omega-\sin \Omega \cos i \cos \omega}{-\frac{X}{Y} \sin \Omega \sin \omega+\frac{X}{Y} \cos \Omega \cos i \cos \omega+\cos \Omega \sin \omega+\sin \Omega \cos i \cos \omega}\right] .
$$

We now have an analytical expression for $\nu$ solely as a function of the KOE, $X$, and $Y$ of a particular point on the orbital ellipse. 
By combining the original $X$ and $Y$ equations to solve for $\nu$ analytically, we have created two potential solutions at $\nu$ and $\nu \pm \pi$. One of these is the correct $\nu$ value and the other is not. To calculate the correct intersection point, we calculate the separations at both $\nu$ and $\nu \pm \pi$ to find absolute error in $s$ and use the smaller error of the two.

\subsection{Calculate $t$ from $\nu$}

We have $\nu$ for the locations where $s$ extrema, $\Delta$ mag extrema, $s_{\mathrm{WA}}$-orbit intersections, and $\Delta$ mag intersections occur, but we need them in terms of time. We calculate eccentric anomaly $E$ of these events directly from $\nu$ as

$$
E=\tan ^{-1}\left(\frac{\sqrt{1-e^{2}} \sin \nu}{e+\cos \nu}\right)
$$

which gives the corresponding time

$$
t=\frac{E-e \sin (E) T}{2 \pi},
$$

where $T$ is the orbital period of a general planet.

\subsection{Converting from Star to Star}

The planet-star intersection points and visibility ranges can be saved as either true anomalies or as specific times for a reference star. It makes the most sense to store the values as times and use $1 M_{\odot}$ as the reference time. The times are scaled to any star mass $M$ by

$$
T=T_{\odot} \sqrt{\frac{M_{\odot}}{M}} .
$$

\subsection{Calculating Completeness}

We calculate integration time adjusted completeness using the aggregated fraction of time that planets are detectable. Using the methods described in Sec. 2 on the instrument's photometric visibility limit $\left(\Delta \mathrm{mag}_{\text {lim }}\right)$ and astrometric visibility limits (IWA and OWA), we calculate the specific times that the planet enters or exists the instruments visibility limits. By collecting these times and inspecting intermediate test points, we identify the time windows in which any planet is detectable by the instrument. Given an integration time $\left(t_{\max }\right)$ required to reach some SNR consistent with a clear detection (typically a value $>5$ ), we throw out all visibility time windows less than $t_{\max }$ and discount all other visibility windows by $t_{\max }$. Dividing the sum of all integration time discounted visibility windows for a planet by its orbital period gives us the fraction of time that a planet is detectable by the instrument. Aggregating the fractions of time that planets are detectable by the instrument gives us integration time adjusted completeness. In mathematical form, this is given as

$$
C_{t_{\max }}=\frac{1}{N}\left(\sum_{\forall k} \frac{\sum_{\forall j}\left(\delta t_{j, k}-t_{\max } U_{k}\right)}{T_{k}}\right) .
$$

Here $\delta t_{j, k}$ is the $j$ 'th time window larger than $t_{\max }$ in which the $k^{\prime}$ th planet is visible, $U_{k}$ is a boolean that indicates that the planet is always visible 0 or at least sometimes visible $1, N$ is the total number of planets, and $T_{k}$ is the orbital period for the $k^{\prime}$ th planet.

We also calculate the completeness for an Earth-like exoplanet of the Earth-like exoplanets subpopulation:

$$
C_{\oplus, t_{\max }}=\frac{1}{N_{\oplus}}\left(\sum_{\forall k \in \oplus} \frac{\sum_{\forall j}\left(\delta t_{j, k}-t_{\max } U_{k}\right)}{T_{k}}\right),
$$

where $N_{\oplus}$ is the total number of Earth-like planets simulated. 
Similarly, this capability can be extended to the entirety of the Kopparapu classification scheme. ${ }^{4}$ Our method uses orders of magnitude fewer exoplanets and an equivalent memory but fills in the $\Delta$ mag space using an order of magnitude better accounting with strategically calculated true anomalies.

\subsection{Calculating Dynamic Completeness}

The methods presented in this paper can also be used to calculate dynamic completeness. ${ }^{13}$ Dynamic completeness extends the original concept of completeness by considering the fraction of planets observable on subsequent observations of the same target. For the original formulation of completeness, which relies on simulating a cloud of planets, this requires propagating every simulated planet along its orbits. Dynamic completeness is frequently used to compute the fraction of planets in a population that are initially undetectable but become detectable upon a second observation some time later.

We define $\mathbf{P}$ as the unordered set of all planets, $\mathbf{P}_{\text {detected, } 1}$ as the set of planets detected at observation time one, and $\mathbf{P}_{\text {undetected, } 1}$ as the set of planets not detected at observation time one. To find the sets of detected planets at the first observation, we start by generating a random observation time for the $k^{\prime}$ th planet $\left(t_{\text {start }, k}\right)$ between 0 and $T_{k}$. We then determine which planets are within the instrument's visibility limits at $t_{\text {start }, k}$ to create $\mathbf{P}_{\text {detected, } 1}$ and its complement $\mathbf{P}_{\text {undetected, } 1 \text {. }}$.

For a subsequent observation some time later $\left(t_{\text {wait }}\right)$, we find the time past periastron at which the observation occurs by $\left[\bmod \left(t_{\text {start }}+t_{\text {wait }}, T_{k}\right)\right]$ and determine which planets are within the instrument's visibility limits at that time. This defines the unordered set of planets detected at observation time two as $\mathbf{P}_{\text {detected,2. }}$. Its complement is $\mathbf{P}_{\text {undetected,2 }}$, the set of planets not detected at observation time two.

Dynamic completeness for the second observation of a target star is given by the fraction of planets undetected in the initial observation but detected on the second one:

$$
C_{2}=\frac{1}{N} n\left(\mathbf{P}_{\text {undetected }, 1} \cap \mathbf{P}_{\text {detected }, 2}\right),
$$

where $n(\mathbf{P})$ is a function giving the number of elements in set $\mathbf{P}$ and $N$ is the total number of planets.

Dynamic completeness of the $m$ 'th visit is similarly given by the fraction of previously undetected planets that are detected on that visit:

$$
C_{m}=\frac{1}{N} n\left(\left(\bigcap_{j=0}^{m-1} \mathbf{P}_{\text {undetected, } \mathrm{j}}\right) \cap \mathbf{P}_{\text {detected, } \mathrm{m}}\right) .
$$

\section{Results}

\section{$3.1 \nu$ from $s$}

We apply the methods developed Sec. 2 to calculate the planet-star separation extrema, the true anomalies at which they occur, the times past periastron at which the extrema occur, the true anomalies where the separation circle intersects with the projected orbital ellipse, and the times at which these intersections occur for a planet orbit. Figure 9 shows the projected separation of a sample orbit with global and local extrema calculated via Sec. 2 methods. This figure also demonstrates the ability of our methods to find all true anomalies (and times) when the projected separation takes a specific value (in this case $s_{\mathrm{WA}}=1 \mathrm{AU}$ ).

We now determine the accuracy of the methods implemented. To do this, we calculate the true anomalies of $s_{\mathrm{WA}}$-orbit intersections for $10^{5}$ orbits using the method in Sec. 2.1.3 and $s_{\mathrm{WA}}=$ $1 \mathrm{AU}$. We then calculate the planet-star separation by substituting these true anomalies back into Eq. (2) and plot the error between these separations and the input $s_{\mathrm{WA}}$ in Fig. 10. Of the $10^{5}$ planets orbits simulated, $\sim 25,000$ orbits produced two or more intersections. The largest 
intersection error observed is $<10^{-6}$. Machine precision error is $\sim 10^{-16}$, and the square root of this is $10^{-8}$, which indicates that we have approached machine precision in our method.

\section{$3.2 \nu$ from $\Delta$ mag}

We apply the methods developed Sec. 2 to calculate the $\Delta$ mag extrema, the true anomalies at which they occur, the times past periastron at which the extrema occur, the true anomalies where the $\Delta$ mag intersections occur, and the times at which these $\Delta$ mag intersections occur for a planet orbit. Figure 11 shows the $\Delta$ mag of a sample orbit with global and local extrema calculated via Sec. 2 methods. This figure also demonstrates the ability to find all true anomalies (and times) when the $\Delta$ mag takes a specific value (in this case $\Delta \operatorname{mag}_{\text {lim }}=25.0$ ).

To check the error in true anomaly produced by the $\Delta$ mag intersection method in this paper, we compare the $\nu$ of $\Delta$ mag intersections calculated with alternative numerical solving methods. The method presented in this paper finds $\Delta$ mag intersections of $10^{6}$ planets within $\sim 6.3 \mathrm{~s}$. The first error checking method uses a cubic spline fit to $300(\nu, \Delta \mathrm{mag})$ points along each planet's orbit. We then subtract $\Delta \operatorname{mag}_{\text {lim }}=29$ (our test point) from the spline and find the roots. This univariate spline root solving method is capable of executing in $419 \mathrm{~s}$ on the 510,120 planets in the population that produced two intersections, a rate of $8.1 \times 10^{-4} \mathrm{~s}$ per planet. The univariate spline root solving method is $100 \times$ slower than the $\Delta$ mag intersection method in Sec. 2.2. The separation error between the univariate spline method and the $\Delta$ mag intersection method is $<6 \times 10^{-5}$ for $>99.98 \%$ of planets and $<10^{-4}$ for the other $0.02 \%$. These high-error targets are planets with low variation in $\Delta \operatorname{mag}(\nu)$ orbits. The second-error checking method solves for the $(\nu, \Delta \mathrm{mag})$ intersections using a numerical minimization method on a random subset of $10^{4}$ planets. This method is far more inefficient, taking $1646 \mathrm{~s}$ on $10^{4}$ planets with two intersections at a rate of $0.1646 \mathrm{~s}$ per planet. Using a minimization function allows us to determine the error in $\Delta$ mag of an intersection point to within $10^{-8}$. This numerical minimization method independently confirms the accuracy of solutions to the univariate spline roots method on the limited number of targets tested. We plotted the normalized frequency of true anomaly error of both the numerical minimization method and univariate spline root solving method compared against the $\Delta$ mag intersection method in Fig. 12. Note that the total number of incidences of a given error is normalized by the bin width and total number of targets, so the non-normalized frequency of the $3 \times 10^{-11}$ bin is incredibly large compared with the $5 \times 10^{-4}$ bin. Both methods indicate that the resulting true anomalies of the $\Delta$ mag intersections are within $10^{-4} \mathrm{rad}$ of each other.

Although the $\Delta$ mag intersection method featured in this paper is orders of magnitude faster than the univariate-spline-roots method and just as accurate, the quasi-Lambert phase function required for its use is not the best phase function for all planets; however, it does fit Earth-like planet phase function quite well. At a substantial time cost, the univariate-spline-roots method can be used for planets with any phase function.

\subsection{Convergence and Validation}

We test convergence of our method for calculating completeness by repeatedly calculating completeness on $10^{5}$ planets. We test convergence of Brown's method by repeatedly calculating completeness over a set of a logarithmically increasing number of planets. We use the same SAG13 planet population parameters from Ref. 1 but make the substitution of a quasi-Lambert phase function to define the underlying planet population. Since this technique will be most relevant to future highly capable telescopes, we are using the HabEx IWA of $0.045 \mathrm{arc} \mathrm{sec}$, OWA of 6 arc sec, and upper $\Delta$ mag limit of 25 on a star that is $10 \mathrm{pc}$ away. ${ }^{8}$ This resulting instrument visibility limits and planet population cover a well populated region of the $\Delta$ mag versus $s$ joint probability density function [see Fig. 1 in Ref. 1 for a comparable example of the joint probability density function]. Convergence of the two completeness methods is demonstrated in Fig. 13.

Both methods presented in Fig. 13 demonstrate similar percent convergence to their own converged means. Completeness repeatedly calculated using the method in Sec. 2.6 with $10^{5}$ planets has a mean of 0.25785 with a standard deviation of 0.0010 for $4.28 \times 10^{8}$ planets. In comparison, the Brown completeness method converges to 0.25783 . This error in the converged 
mean of the two methods is consistent with the completeness error identified in Fig. 14. We expect that completeness calculated using the method in this paper will have comparatively better convergence in sparsely populated regions of the $\Delta$ mag versus $s$ joint probability density function (see Fig. 1 in Ref. 1).

Since completeness for an individual planet is the fraction of time that a planet is visible to the instrument, we validate individual planet visibility windows using test points. To calculate a ground truth fraction of time that a planet is visible, we create $10^{5}$ test points, evenly spaced in time, and calculate both the planet-star separation and planet-star $\Delta$ mag at each of theose test points. We then determine whether each point is within the visibility limits of the instrument. The fraction of visible points is the fraction of time that a planet is visible. We repeated this for 25,000 planets due to computational cost of the test point method. The histogram of error in completeness for individual planets calculated using the test point method and method in this paper is shown in Fig. 14. The maximum error in completeness is observed to be near $5 \times 10^{-5}$, which is consistent with the converged error between Brown completeness and integration time adjusted completeness.

The test point method that we used to validate integration time adjusted completeness calculations took over $52 \mathrm{~h}$ on only 25,000 planets (less than a quarter of the planets needed to compute completeness using integration time adjusted completeness). The computation time for the integration time adjusted completeness calculation on $10^{5}$ planets is $21.34 \mathrm{~s}$ with a standard deviation of $0.4 \mathrm{~s}$ over 1000 calculations. Brown completeness, including the joint probability density function generation, has a mean execution time of $3.19 \mathrm{~s}$ and standard deviation of $0.13 \mathrm{~s}$ over 1000 calculations. This execution time of all of these methods scales linearly with the number of planets. Some room for optimization exists in integration time adjusted completeness.

\subsection{Completeness Versus Integration Time}

To evaluate the effects of integration time on completeness, we calculate integration time adjusted completeness for various integration times, star distances, and planet populations (SAG13 and Earth-like planet population). Figure 15 shows the integration time adjusted completeness for the SAG13 planet population ${ }^{1}$ and the Earth-like planet subpopulation defined in Appendix L. The decrease in completeness for longer integration times is most prominent for nearby stars for both populations. As expected, longer integration times decrease completeness.

For the assumed observatory parameters, the Brown completeness of an Earth-like population for a target $5 \mathrm{pc}$ away is 0.583 . As we increase integration time to 1,2 , and 5 days, the associated integration time adjusted completeness decreases by $0.63 \%, 1.27 \%$, and $3.15 \%$, respectively. Recalculating Brown completeness for a target at $25 \mathrm{pc}$ in Fig. 15(b) gives 0.583. As we increase integration time to 1,2 , and 5 days on this $25 \mathrm{pc}$ target, the associated integration time adjusted completeness decreases by $0.989 \%, 1.97 \%$, and $4.92 \%$, respectively. Our integration time adjustment of completeness shows Brown completeness overestimates exoplanet yields for any observation. Brown completeness applied to stars farther away results in a substantial overestimation of exoplanet yield.

Technically, the $\Delta \operatorname{mag}_{\text {lim }}$ used as the upper limit for calculating completeness is a function of integration time and will approach a theoretical upper limit as the noise floor is reached. By calculating $\Delta$ mag $_{\text {lim }}$ using Eq. (12) of Ref. 1, we can calculate completeness at each integration time to get the completeness versus integration time curve. We created a 4-m telescope as in Appendix $\mathrm{M}$ and evaluated completeness at each integration time to create an example demonstrating how Brown completeness and integration time adjusted completeness vary with integration time in Fig. 16. We can see that the completeness of both methods tracks very closely until they diverge. Brown completeness approaches its asymptotic limit as expected, whereas integration time adjusted completeness approaches a maximum and begins decreasing past $\sim 0.2$ day.

\subsection{Dynamic Completeness}

We compute dynamic completeness for the example test case in Fig. 1 of Ref. 13 using the method described in this paper and replicating the approach of the original work. This computation is only for a second epoch. We replicated the original work by sampling a large number of planets, finding the planets initially visible, propagating these planets to some time past initial 
observation, and determining what fraction of these planets were not detected the first time but were detected the second time. We replicated Brown's work using both the Lambert phase function (orange line in Fig. 17) to match the original work as well as the quasi-Lambert phase function (red line in Fig. 17).

We compared the dynamic completeness computation time for 1000 dynamic completeness calculations testing 1000 individual points in time past the initial observation. Brown's dynamic completeness had an average execution time of $34.62 \mathrm{~s}$ with a standard deviation of $0.99 \mathrm{~s}$. Calculating dynamic completeness using the method presented in this work resulted in an average execution time of $4.757 \mathrm{~s}$ with a standard deviation of $0.036 \mathrm{~s}$. The dynamic completeness calculation presented in this paper is $\approx 7 \times$ faster than the traditional Brown completeness method, enabling its use in optimization.

\section{Discussion}

\subsection{Convergence}

Both methods of calculating completeness suffer from sparse sampling of the exoplanet population parameter space. Brown completeness is most affected as it needs planets sampled over $\Omega, \omega, a, e, i, \nu, p$, and $R$. The current implementation of our method needs the same parameters except for $\nu$. We are therefore surprised that Brown's method and our method share such similar completeness convergence. We hypothesize that the similar convergence results are because the integration bounds used for calculating completeness are over a dense region of the joint probability density function. If we examined a more sparse region of the joint probability density function such as high $\Delta$ mag or larger separation (where the larger, lower occurrence rate planets occur), we expect our completeness methods to have marginally better convergence for low numbers of planets. Regardless, integration time adjusted completeness easily reaches completeness errors below $10^{-3}$ (translates to $\approx 0.8 \%$ where $C=0.12$, the largest completeness of an observation in Ref. 1), which is a sufficient error for use in optimization.

\subsection{Reducing Parameter Spaces}

The integration time adjusted completeness method implemented in EXOSIMS is not cached for computational efficiency like the Brown completeness is. Although we are able to store the joint probability density function of $\Delta$ mag versus $s$ with Brown's method, integration time completeness requires the KOE of each simulated planet to be stored. The curse of dimensionality prohibits us from finely sampling the entire subspace of exoplanets, making the caching of integration time adjusted completeness prohibitive, but not all our methods require all $6 \mathrm{KOE}$, $p$, and $R$. So, in the future, it may be possible to create a representative subsampling of planets weighted by occurrence rate based on the parameters needed for different calculations. For example, $s$ extrema, $s_{\mathrm{WA}}$-orbit intersections, and $\Delta$ mag extrema calculations only require $\omega$, $a, e$, and $i$ (Note that the $\nu$ locations of the $\Delta$ mag extrema are independent of $p$ and $R$, but the magnitude of $\Delta$ mag extrema are dependent upon them). The photometric property $p \times R$ could then be sampled and independently combined with individual $(\omega, a, e, i)$ combinations. The primary benefit is that, given some instrument parameters and star properties, we could determine the subset of parameters that can physically be observed prior to calculating completeness, thus making the per-star completeness calculations more efficient.

\subsection{Limitations}

The approach we implemented in this paper did not vary the $\Delta \operatorname{mag}_{\text {lim }}$ as we varied the integration time. If properly executed, $\Delta \mathrm{mag}_{\mathrm{lim}}$ should increase with increased integration time. However, calculating the planet true anomaly intersections with a given $\Delta \operatorname{mag}_{\text {lim }}$ is the most expensive calculation, so repeatedly calculating this to convergence is not desirable.

The $\Delta \operatorname{mag}_{\text {lim }}$ of coronagraph designs are working angle dependent and therefore separation dependent, but we assume that this limit is a fixed quantity. If there is substantial variation in the 
limiting $\Delta$ mag, it is better to use the conservative value with this method. If a higher level of predictive accuracy is desired, these methods could be used to ascertain if any intersection with the $\Delta$ mag $_{\text {lim }}$ exists and subsequently another method could be used on the subset of planets with known intersections.

\subsection{Impact of the Integration Time Adjusted Completeness}

As we showed in this paper, integration time adjusted completeness and Brown completeness converge to the same value to within 0.00002 when evaluated at $t_{\max }=0$ day. The integration time adjusted completeness for an observation of an Earth-like planet population on a 1- $M_{\odot}$ star 5 pc away and integration time of $\approx 1$ day is $0.64 \%$ lower than the comparable Brown completeness. For reference, the Roman target list in Table 9 of Ref. 1 has a maximal integration time of 1.71 days but most are $<0.6$ day. This means that we could expect an average reduction in yield below $0.64 \%$ when observing a population of Earth-like planets around Sun-like stars. This integration time completeness adjustment is within the 3.19\%, 3\%, margin of error from 1000 Monte Carlo simulations of the cycle 6 Roman in Ref. 1 and cannot be considered statistically significant. However, a $~ 4$-day integration time on a Sun-like star $25 \mathrm{pc}$ away observing an Earth-like population has a decrease in completeness above the Ref. $13 \sigma$ margin of error. However, the threshold of statistical significance should not deter the widespread use of integration time adjusted completeness as the adoption of this method can shore up the differences between completeness-based yields and simulation-based yields. We can also say that integration time has a muted effect when observing stars that are farther away. This can most likely be attributed to the increase in $s_{\min }$ and resulting decrease in the total time-fraction that smaller period planets spend within the visible limits of the telescope. Big planets with the orbital radius of Jupiter will move more slowly and be less affected by integration times.

In Appendix M, we optimized an exoplanet direct imaging mission maximizing single-visit Brown completeness yield and included the resulting Design Reference Mission (DRM) in Table 2. The resulting DRM has a Brown completeness yield of 387.16 exoplanet detections in a single-visit detection survey on average, but the integration time adjusted completeness yield expects only 354.67 exoplanets on average. This means that Brown completeness for the particular instrument parameters optimized over the SAG13 planet population with the particular mission parameters overpredicts the actual exoplanet yield by 32.49 exoplanets on average, 9.16\% more than the integration time adjusted completeness yield. When calculating completeness, we are careful to scale the completeness limits of integration by the star's luminosity. For the integration time adjusted completeness method, we additionally scale the planet's orbital periods based off the mass of the host star. The percentage difference above and beyond that expected from Fig. 15 can be found by looking at these two adjustments applied to each star and the target list in Table 2. The average star in the target list has a larger mass and a brighter luminosity than that of the Sun. The brighter luminosity results in a smaller $s_{\min }$ and $s_{\max }$, which serves to incorporate more smaller semimajor axis planets into the completeness calculations, and the larger star masses result in shorter periods, meaning that the planet visibility windows all decrease in duration.

Integration time adjusted completeness is crucial for determining the ability of an Earth-like planet to be spectrally characterized. A spectral characterization with a coronagraph could take between a few days and 60 days. Because of the long integration time, the planners of a HabEx use the "characterization completeness" of $10 \%$ and maximum integration time of 60 days as a filter on stars to consider observing. ${ }^{8}$ The calculation of this critical filter could be substantially improved by considering integration time adjusted completeness if the spectrum of the planet must be taken all at once (i.e., observation spanning multiple weeks) and not spread across multiple epochs. Just because a planet can be detected does not mean that it can be spectrally characterized.

\subsection{Dynamic Completeness and Computation Cost}

The greatest benefit of using the methods in this paper to calculate completeness is the marginal additional cost of calculating dynamic completeness. Generally, dynamic completeness requires 
the computation of true anomalies from time, which is prohibitive for $>10^{5}$ orbits at $>100$ different times in the future. With our methods, calculating visibility windows allows us to use boolean operations to compute dynamic completeness about $7 \times$ faster than Brown's dynamic completeness method. The computational cost of calculating completeness using the method described in this paper is an order of magnitude larger than Ref. 2, but it can use orders of magnitude fewer planets to do so. Unlike Brown's method, within the computation time of our method, we also get additional desirable information about the detectable planets such as their $s$ extrema and $\Delta$ mag extrema.

\subsection{Revisiting the Same Exoplanet}

Another limitation present in the planning of exoplanet direct-imaging missions is the telescope keep-out angles. Figure 3 of Ref. 1 contains a keep-out map for a subset of the DRM created in that paper. The smallest percent of time that a target star is visible for the Roman is nominally $28 \%$. Due to symmetry of the keep-out region, this translates into two separate time windows of visibility of $\sim 51$ days. Instead of considering the integration time an integration time input, it can also be considered a revisit time input for determining the probability of being able to observe a planet twice in the same target-star visibility window.

\subsection{Exoplanet Classification}

The underlying methods in this paper are used to find locations along a planet's orbit where $s$ and $\Delta$ mag intersections occur. The methods in this paper, with some modification, can also be used to probabilistically classify an exoplanet subtype. ${ }^{4}$ If an exoplanet is detected with a particular $(s, \Delta \mathrm{mag})$ and an uncertainty region of $s \pm \sigma_{s}$ and $\Delta \mathrm{mag} \pm \sigma_{\Delta \mathrm{mag}}$, then the methods in this paper can be applied to each of these four bounding lines. By finding the average orbital time-fraction that exoplanets of each type spend in the bounding uncertainty box, we can find the probability that the exoplanet belongs to a specific exoplanet subtype. This requires additional work beyond the scope of this paper.

\section{Conclusion}

We have demonstrated an accurate method for calculating integration time adjusted completeness and its adaptation to calculating dynamic completeness. In the process, we also created fast and accurate methods for calculating the true anomalies where a planet's orbit has specific values of projected separation $\Delta$ mag and their extrema. We demonstrated how to use these methods to calculate a more accurate integration time adjusted completeness using the fractions of time that a planet is detectable by an instrument. We demonstrate that traditional methods of calculating completeness overestimate the number of observable planets because they do not subtract the integration time used in observing the target. For a Sun-like star at $25 \mathrm{pc}$ with 1-day and 5-day integration times, integration time adjusted completeness of Earth-like planets is reduced by $1 \%$ and 5\%, respectively. We applied integration time adjusted completeness to a target list optimized using the Brown completeness method and found that Brown completeness overestimated yields by $9.61 \%$. We also demonstrated that our methods can be used to quickly calculate dynamic completeness for determining the optimal time to revisit a target star.

\section{Appendix A: Common Notation}

There are many common variable forms used in this paper that are simply summarizable. Any variable $\underline{x}$ refers to a 3D vector in $X, Y$, and $Z$ coordinates of something. We use $\mathbf{x}$ to indicate an array of variables, specifically used when referring to multiple roots of a polynomial. $|\overline{A B}|$ refers to the length of line segment $\overline{A B}$. Line segment $\overline{A B}$ is treated as a vector. $C^{\prime}$ is a projection of point $C$.

There are multiple different subscripts with different meanings used in this paper. A subscript with $x_{i}$ refers to the index of a host star (out of some whole, non-descript, star catalog). 
A subscript $x_{k}$ refers to an individual planet out of the whole large set of planets. Subscripts of $x_{\min }, x_{\max }, x_{\operatorname{lmin}}$, and $x_{\operatorname{lmax}}$ are descriptors on the individual variable $x$ that indicate that the variable is associated with the minimum, maximum, local minimum, or local maximum, respectively. When solving for the distance between points inside an ellipse and the vertices or covertices of that ellipse with semimajor axis $a$, we use the shorthand notation of the form $s_{a+x, y}$. [This is specifically the distance between point $(x, y)$ and $(-a, 0)$.]

We denote coefficients of the polynomials of the four methods that solve the quartic in this paper as $A_{\#}$.

In Fig. 4, we reference many points on the 3D elliptical orbit and $2 \mathrm{D}$ projection of this orbit into the plane of the sky. Points on the original 3D ellipse are labeled $B, H, P, C, K, O, D, A, B^{\prime}$, $H^{\prime}, P^{\prime}, C^{\prime}, K^{\prime}, O^{\prime}, D^{\prime}$, and $A^{\prime}$.

In Appendix K, we use $p_{0}$ to $p_{11}$ as intermediate constants for simplifying the full quartic expression. $P, D$, and $\Delta$ (by itself and only in this section) are intermediate constants derived from quartic coefficients for determining the sign of the quartic solutions.

$K_{0}$ to $K_{9}$ are intermediate simplifying coefficients used in representing Eqs. (13)-(15) in Sec. 2.1.1. The full expansion of these equations is included in Appendix E.
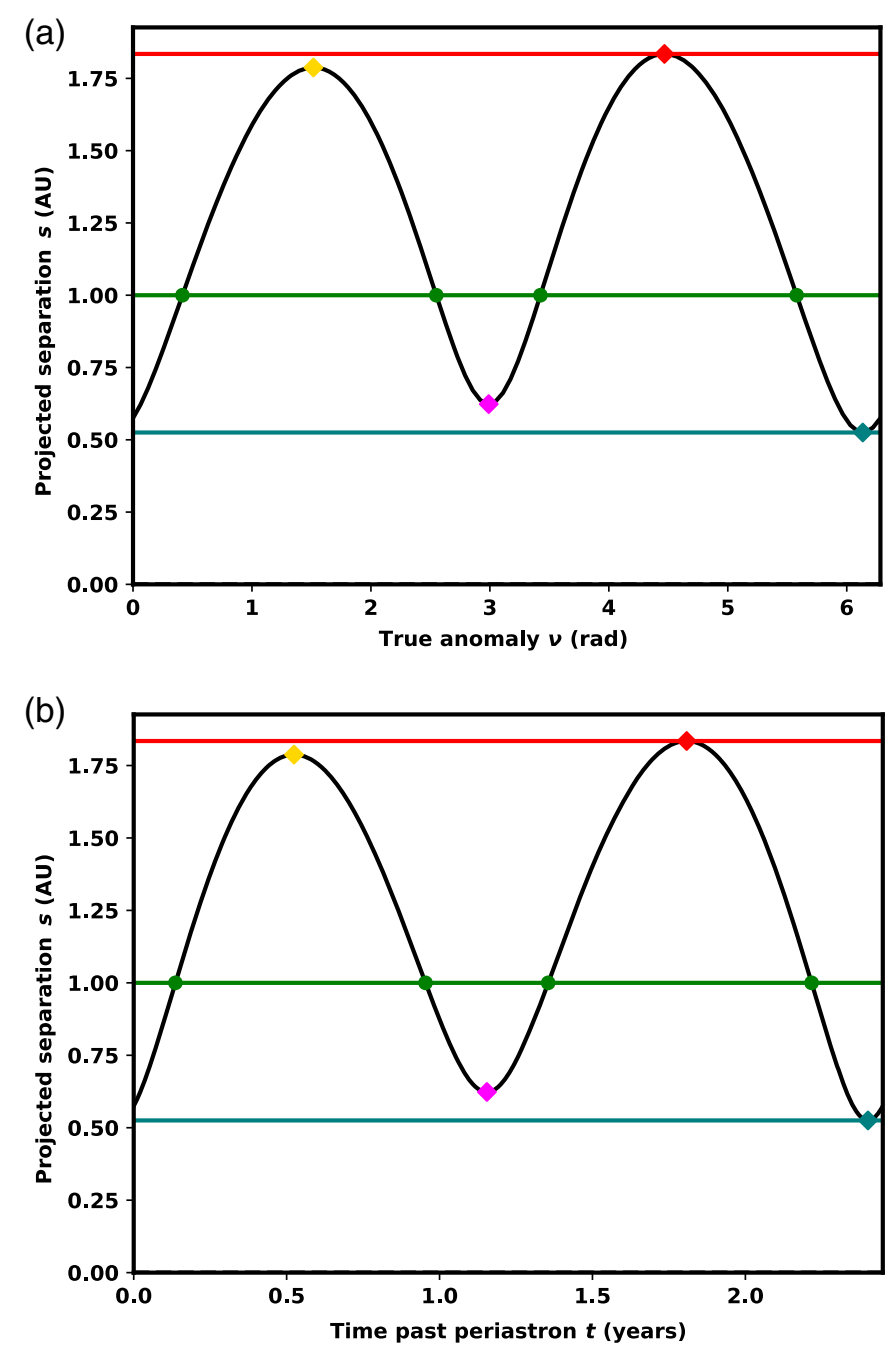

Fig. 9 Planet-star separation of the planet is plotted in black. The separation extrema are indicated by diamonds, where the maximum is red, local maximum is yellow, local minimum is magenta, and minimum is teal. Lines are drawn at the minimum and maximum separations. For the input separation of $s=1 \mathrm{AU}$, the green dots are the analytically calculated orbit intersections. (a) The separation versus true anomaly and (b) the separation versus time past periastron for a planet orbit with $a=1.82 \mathrm{AU}, e=0.09, \Omega=3.37 \mathrm{rad}, \omega=4.86 \mathrm{rad}$, and $i=1.25 \mathrm{rad}$. 


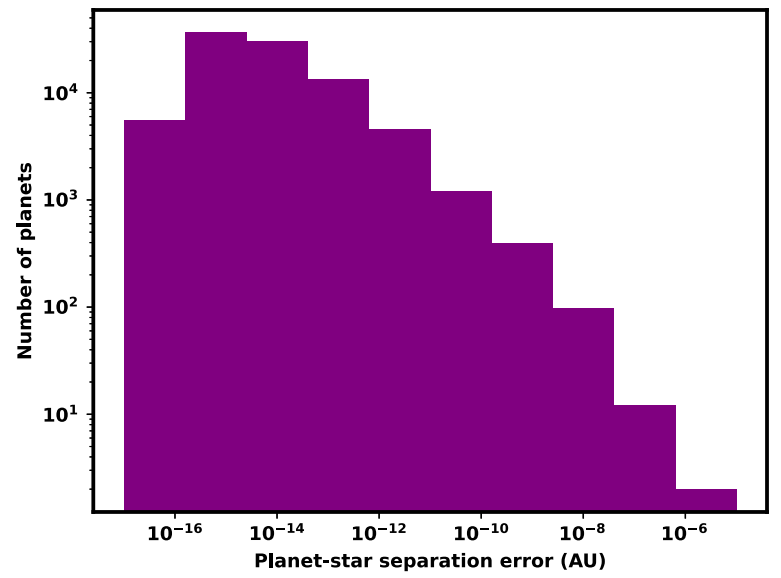

Fig. $10 s_{\mathrm{WA}}$-orbit intersection error histogram calculated for a separation of $s_{\mathrm{WA}}=1 \mathrm{AU}$ for $10^{5}$ planet orbits generated from the SAG13 planet population. Of these $10^{5}$ planet orbits, 6201 orbits have two intersections with the $s_{W A}$ circle, and 17,952 have four intersections with the $s_{W A}$ circle. This results in a total of 84,210 planet-star intersections. After calculating and identifying true anomalies of intersections using the methods described in this paper, we evaluated the planet-star separation of each orbit at the true anomalies and calculated error from the input $s_{\text {WA }}$.
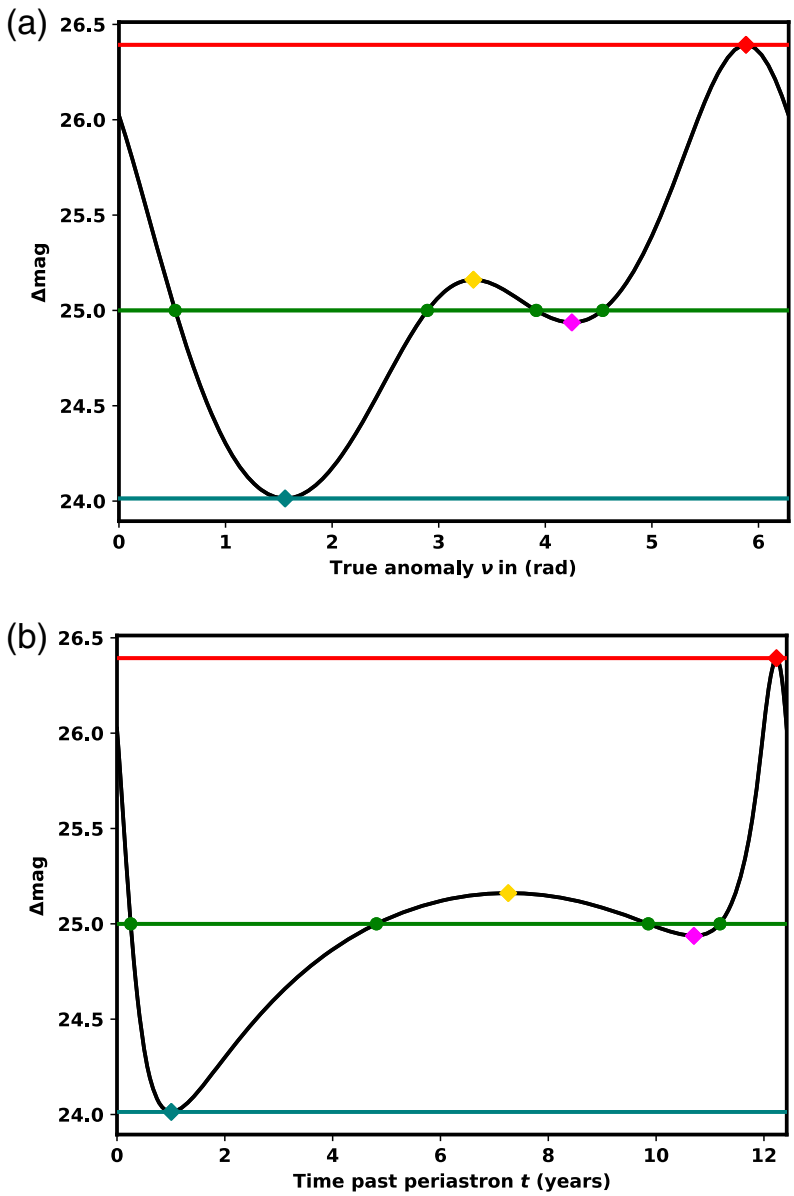

Fig. 11 (a) The $\Delta$ mag of a planet (black line) plotted against $\nu$ and (b) time past periastron. $\Delta$ mag extrema are indicated by diamonds, where the maximum is red, local maximum is yellow, local minimum is magenta, and minimum is teal. Separation minimum and maximum are indicated by horizontal lines. For an input $\Delta$ mag $_{\mathrm{lim}}=25$ (green line), we calculated the specific true anomalies (green dots) where the planet's $\Delta$ mag intersects this line. The specific planet KOE are $a=5.36 \mathrm{AU}, e=0.56, \omega=5.06 \mathrm{rad}, \Omega=0.69 \mathrm{rad}, i=0.81 \mathrm{rad}), p=0.3$, and $R=4 R_{\oplus}$. 


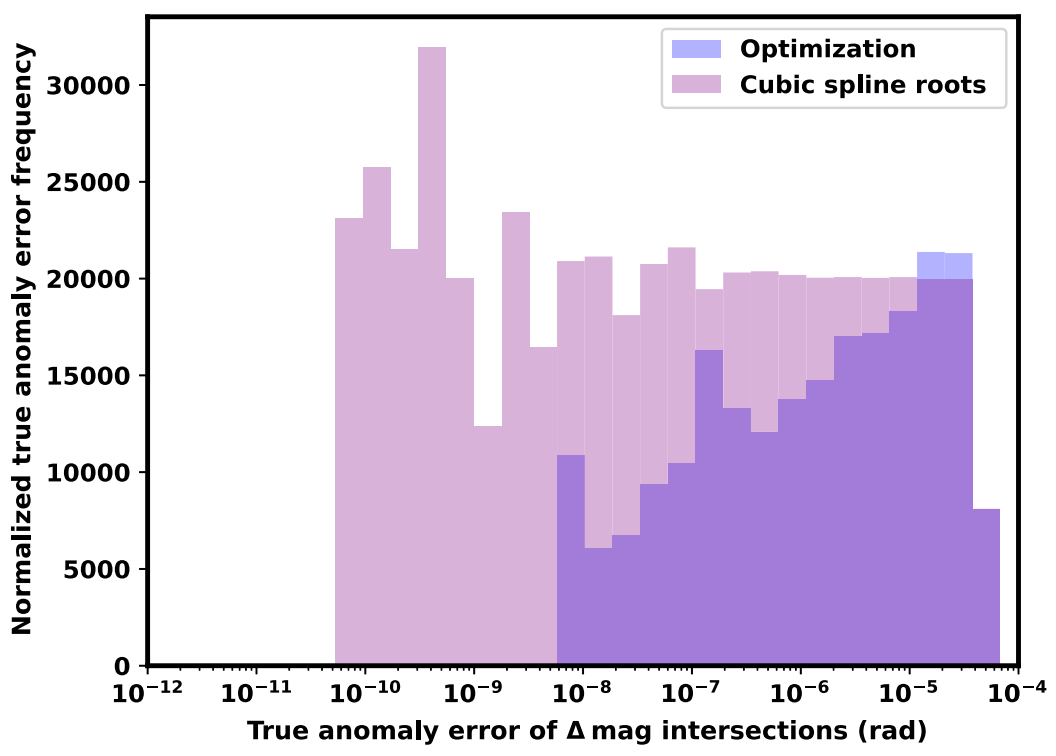

Fig. 12 A histogram of $\nu(\Delta \mathrm{mag})$ error between the root solving method described in this paper and a root solver of a cubic spline fit method (purple) for 510, 120 planet orbits that produce two intersections (totaling 1,020,240 datapoints). We also compare the error between the root solving method described in this paper and an optimization method (blue) for $10^{4}$ planet subset of the planet orbits that produce intersections. The optimization method investigates fewer planets because it is orders of magnitude more computationally expensive, but it is a fundamentally different approach to validating our method than a root solver.

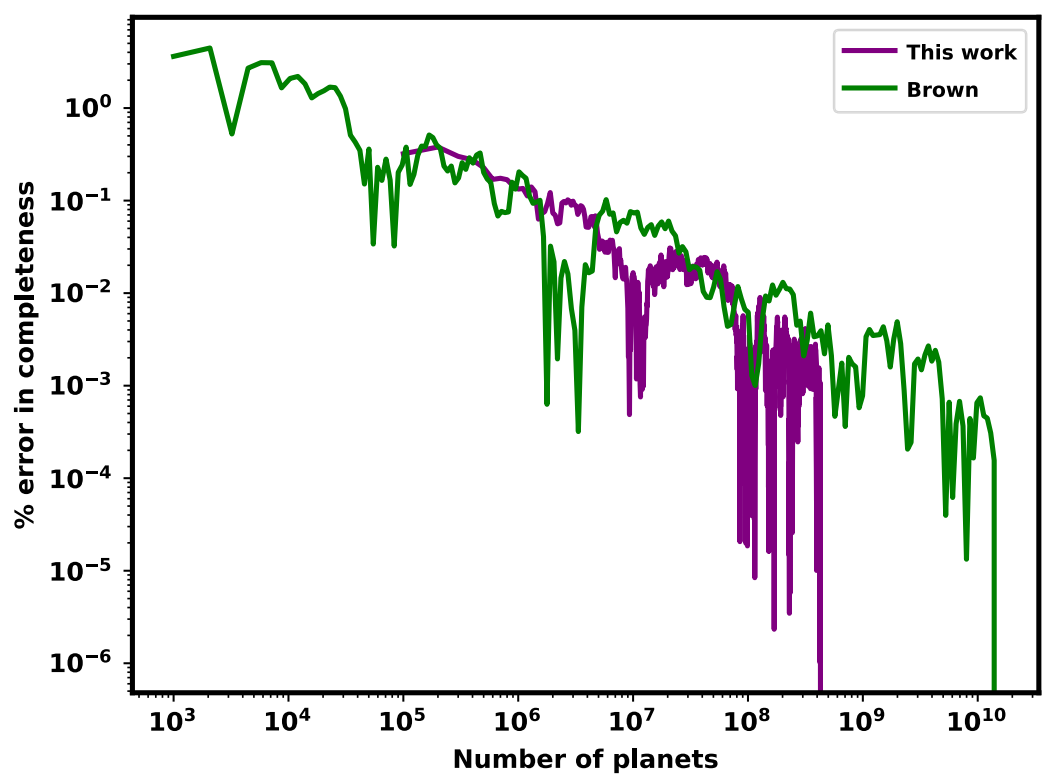

Fig. 13 Convergence of \% error in completeness for varying numbers of planets used in its computation for Brown's method (green) and the method presented in this work with $t_{\max }=0$ day (purple). Completeness at the maximum number of planets is assumed to be the converged value of completeness of the respective methods. The converged mean of Brown's method is 0.25783 compared with the converged mean of the method in this paper of 0.25785 . The standard deviation of the method in this paper is 0.0010 . 


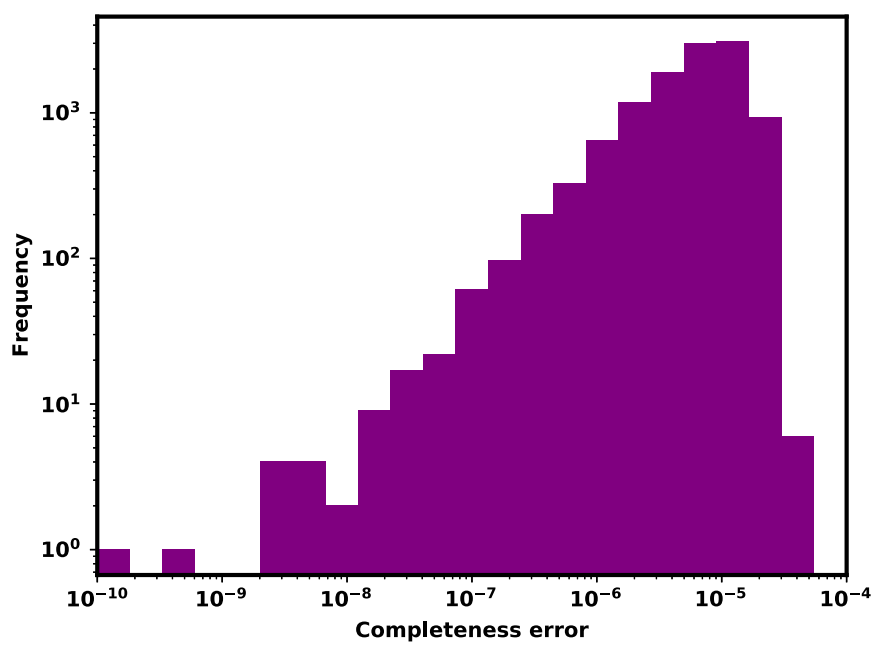

Fig. 14 Per planet completeness error histogram between the integration time adjusted completeness method with $t_{\max }=0$ day and the test point method for 25,000 planets.

(a)

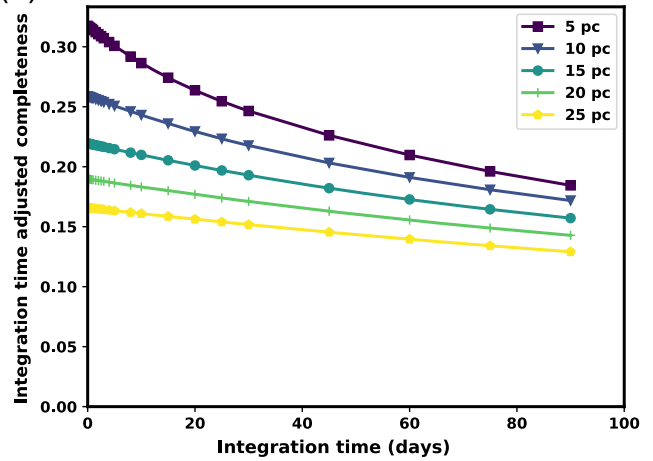

(b)

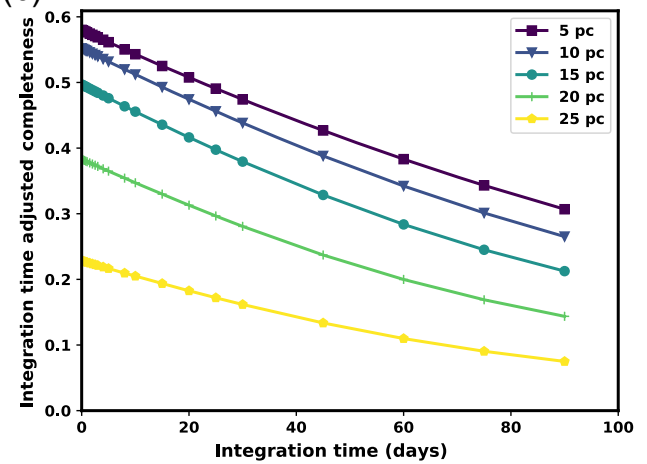

Fig. 15 Integration time adjusted completeness values of (a) SAG13 planet population and (b) Earth-like planet population with IWA $=0.045$ arc sec, OWA $=6$ arc sec, and $\Delta$ mag $=25$ for varying integration times and star distances.

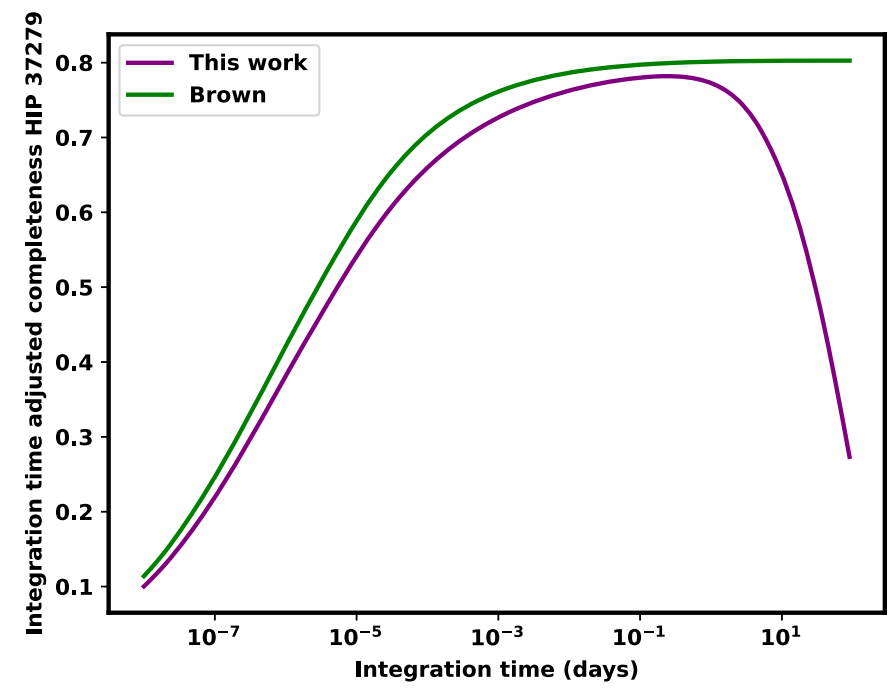

Fig. 16 Brown completeness and integration time adjusted completeness versus integration time for HIP 32,279 assuming a mass of $1.564 M_{\odot}$, luminosity of $7.12 L_{\odot}$, and distance of $d_{i}=3.51 \mathrm{pc}$. The assumed telescope is a 4-m monolith with IWA $=0.045$ arc sec and OWA $=6$ arc sec and $\Delta$ mag $_{\text {lim }}$ computed using the instrument noise model as in Ref. 1. 


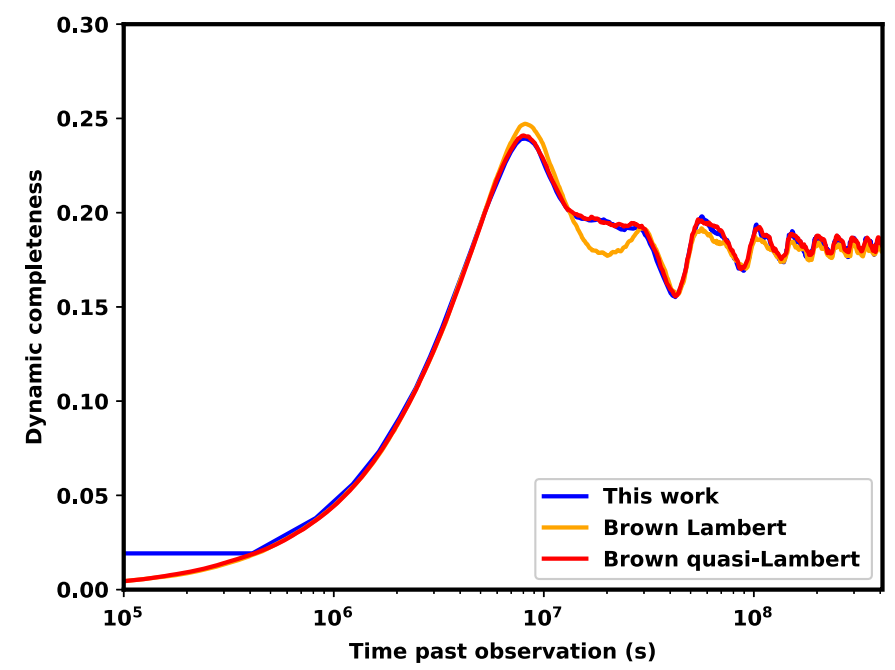

Fig. 17 Dynamic completeness assuming the same parameters as in Ref. 13 ( $\Delta \mathrm{mag}=26$, $M_{\mathrm{HIP} 29271}=1.103 M_{\odot}, d_{i}=10.215 \mathrm{pc}, \mathrm{OWA}=600$ arc sec, IWA $=0.075$ arc sec, $0.7 \sqrt{L} \leq a \leq$ $1.5 \sqrt{L}, 0 \leq e \leq 0.35, p=0.26, R=1 R_{\oplus}$, and $L=0.83 L_{\odot}$ ). In this work (blue), we assume a quasi-Lambert phase function, but in Ref. 13, a Lambert phase function is assumed. Work was done to replicate Brown's original work (Brown Lambert, orange line) and replicate Brown's work using the quasi-Lambert phase function (Brown quasi-Lambert, red line).

\section{Appendix B: True Anomalies of $s$ Intersection Process}

The process outlined here is used in Sec. 2.1.2.

1. Define KOE.

2. $\underline{r}_{k / i}$ from KOE.

3. Orbiting foci $F$ from KOE.

4. Calculate 3D orbit ellipse center.

5. Project 3D ellipse to 2D ellipse.

(a) Prove that $3 \mathrm{D}$ ellipses project to $2 \mathrm{D}$ ellipses.

(b) Project 3D ellipse center to 2D ellipse center.

(c) Project ellipse orbiting foci into plane of the sky.

(d) Project semiminor axis line of 3D ellipse to 2D ellipse.

(e) Project semimajor axis line of 3D ellipse to 2D ellipse.

(f) Calculate $\overline{Q Q^{\prime}}$ construction line from semiminor axis and semiminor axis.

(g) Calculate projected ellipse semimajor axis and semiminor axis from $\overline{O^{\prime} Q^{\prime}}$ and $\overline{O^{\prime} Q}$.

(h) Calculate projected semimajor axis angular offset from $X$ axis of plane of the sky.

6. Derotate the projected ellipse.

7. Center the projected ellipse.

8. Find minimum, maximum, local minimum, and local maximum of projected planet-star separation.

(i) Formulate projected planet-star separation equation.

(j) Reformat and set $\delta s^{2} / \delta x_{e}$ equal to 0 .

(k) Combine coefficients into standard quartic form.

(l) Use standard general quartic solutions.

(m) Take the absolute value and only real component of $x_{e}$ solutions.

(n) Calculate $y_{e}$ associated with each solution.

(o) Assign solutions to minimum, maximum, local minimum, and local maximum planetstar separations. 
i. For all solutions (all real and only 2 real):

A. Calculate $s_{\min }$ from $x_{1}$ and assign $\left(x_{\min }, y_{\min }\right)$ of quadrant 1.

B. Calculate $s_{\max }$ from $x_{0}$ and assign $\left(x_{\max }, y_{\max }\right)$ of quadrant 1 .

ii. All real solutions (where all solutions have $\mathfrak{s}(y)<10^{-5}$, additionally assign).

C. The larger of $s_{\left(x_{2}\right)}$ and $s_{\left(x_{3}\right)}$ becomes $s_{1 \max }$ and assign $\left(x_{\operatorname{lmax}}, y_{\operatorname{lmax}}\right)$ of quadrant 1.

D. The smaller of $s_{\left(x_{2}\right)}$ and $s_{\left(x_{3}\right)}$ becomes $s_{\operatorname{lmin}}$ and assign $\left(x_{\operatorname{lmin}}, y_{\operatorname{lmin}}\right)$ of quadrant 1.

iii. Assign solution signs to proper quadrants.

9. Find circle and projected derotated centered ellipse intersection points.

(p) Formulate circle-ellipse intersection equation.

(q) Reformat into standard quartic form.

(r) Use standard general quartic solutions.

(s) Classify intersection solutions.

iv. Inside outer separation bounds, $s_{\min }<s<s_{\max }$.

v. Inside local $\min / \max$ separation bounds, $s_{\operatorname{lmin}}<s<s_{\operatorname{lmax}}$.

vi. Outside outer separation bounds (no intersections).

(t) Calculate intersection solutions for planets with $s_{\min }, s_{\max }, s_{\operatorname{lmin}}$, and $s_{\operatorname{lmax}}$.

vii. Two intersections on same $y$ side of ellipse as the star, $s_{\min }<s<s_{\operatorname{lmin}} x=$ $\left\{\begin{array}{c}x_{3}, \mathfrak{I}\left(x_{1}\right)>10^{-10} \\ x_{1}, \mathfrak{s}\left(x_{1}\right)<10^{-10} \\ x_{0}\end{array}\right.$

viii. Four intersections where, $s_{\operatorname{lmin}}<s<s_{\operatorname{lmax}}$

- Calculate $\Delta x=x_{k}-|x|,(k \in\{0,1,2,3\})$.

- Sort $\Delta x_{k}$ from min to max and rearrange the associated $x_{k}$ to match that order.

- $\quad$ Same $X Y$ points is $x_{3}$.

- Same $X$ opposite $Y$ is $x_{2}$.

- Opposite $X$ Same $Y$ is $x_{0}$.

- Opposite $X$ opposite $Y$ is $x_{1}$.

ix. Two intersections on opposite $x$ side of ellipse as the star, $s_{\operatorname{lmax}}<s<s_{\max }$.

- $x_{0}$ and $x_{1}$.

- $y_{1}$ is opposite sign of $|y|$.

(u) Calculate intersection solutions for planets with only $s_{\min }$ and $s_{\max }$.

x. Calculate projected ellipse quadrant separation bounds $s_{x, b+y}, s_{x, b-y}, s_{a+x, y}$, and $s_{a-x, y}$.

xi. Identify star location type and quadrant order.

- Type 0 occurs where $s_{x+a, y}<s_{x, y+b}$. Smallest to largest order: $s_{x, b-y}, s_{a-x, y}$, $s_{x+a, y}, s_{x, y+b}$.

- Type 1 occurs where $s_{x, y+b}<s_{a-x, y}$. Smallest to largest order: $s_{x, b-y}, s_{x, y+b}$, $s_{a-x, y}, s_{x+a, y}$.

- Type 2 occurs where $s_{a-x, y}<s_{x, y+b}$ and $s_{x, y+b}<s_{x+a, y}$ and $s_{x, b-y}<s_{a-x, y}$. Smallest to largest order: $s_{x, b-y}, s_{a-x, y}, s_{x, y+b}, s_{x+a, y}$.

- Type 3 occurs where $s_{a-x, y}<s_{x, b-y}$. Smallest to largest order: $s_{a-x, y}, s_{x, b-y}$, $s_{x, y+b}, s_{x+a, y}$. 


\section{Appendix C: $\nu$ from $\Delta$ mag Extrema process}

Here we present the full outline of our process for calculating true anomalies where the planet's orbit has a $\Delta$ mag extrema. These steps are discussed in Sec. 2.2.

1. Substitute into Eq. (1) components and expand until all terms are functions of KOE.

2. Manipulate the $\Delta$ mag equation until all $\nu$ terms are isolated on the right side.

3. Take the derivative.

4. Replace $\sin (\nu)$ with $\sqrt{1-\cos (\nu)^{2}}$.

5. Replace all $\cos (\nu)$ with $x$.

6. Isolate all square root terms to general form $\mathbb{A}^{2} \sqrt{1-x^{2}}=\mathbb{B}^{2}$.

7. Square both sides.

8. Find polynomial coefficients of $x$.

9. Solve with root solver.

10. Filter out $\mathbf{x}$ solutions with imaginary components $\left(>10^{-7}\right)\left(10^{-8}\right.$ is approximately $\sqrt{10^{-16}}$, machine precision).

11. Filter out invalid solutions, $1<\mathbf{x}$ or $-1>\mathbf{x}$.

12. Compute $\boldsymbol{\nu}_{0}=\cos ^{-1}(\mathbf{x})$ and $\boldsymbol{\nu}_{1}=2 \pi-\boldsymbol{\nu}_{0}$.

13. Compute associated $\Delta \operatorname{mag}_{0}$ and $\Delta \operatorname{mag}_{1}$.

14. Remove solutions that are not extrema.

15. Assign solutions $\Delta \operatorname{mag}_{\max }=\min \left(\Delta \operatorname{mag}_{0}, \Delta \operatorname{mag}_{1}\right)$.

16. Assign solutions $\Delta \operatorname{mag}_{\min }=\max \left(\Delta \operatorname{mag}_{0}, \Delta \operatorname{mag}_{1}\right)$

17. Remove duplicate solutions.

18. Remove assigned solutions.

19. Check if solution is extrema.

20. Assign $\Delta \operatorname{mag}_{1 \min }$ and $\Delta \operatorname{mag}_{\operatorname{lmax}}$.

\section{Appendix D: $\nu$ from $\Delta$ magi $_{\text {lim }}$ Intersection Process}

Here we present the full outline of our process for calculating true anomalies where the planet's orbit has a specified $\Delta$ mag, which we call an intersection. These steps are discussed in Sec. 2.2.

1. Find $\Delta$ mag extrema.

2. Find planets where $\Delta \operatorname{mag}<\Delta \operatorname{mag}_{\max }, \Delta \operatorname{mag}>\Delta \operatorname{mag}_{\min }$, and $\Delta \operatorname{mag}>\Delta \operatorname{mag}_{\operatorname{lmax}}$ or $\Delta$ mag $<\Delta$ mag $_{\text {lmin }}$ (these planets have only two intersections).

3. Substitute into $\Delta$ mag equation components and expand until all terms are functions of KOE.

4. Manipulate the equation until all $\nu$ terms are isolated on the right side.

5. Expand.

6. Replace $\sin (\nu)$ with $\sqrt{1-\cos (\nu)^{2}}$.

7. Replace all $\cos (\nu)$ with $x$.

8. Isolate all square root terms to general form $A^{2} \sqrt{1-x^{2}}=B^{2}$.

9. Square both sides.

10. Expand.

11. Find polynomial coefficients of $x$.

12. Solve with root solver.

13. Filter out solutions with imaginary components $\left(>10^{-7}\right)$.

14. Filter out solutions $1<x_{k}$ or $-1>x_{k}$.

15. Take arrays $\nu_{0}=\cos ^{-1}(x)$ and $\nu_{1}=2 \pi-\nu_{0}$.

16. Solve for associated arrays $\Delta \operatorname{mag}_{0}$ and $\Delta \operatorname{mag}_{1}$. 
17. Remove solutions where $\left|\Delta \operatorname{mag}_{0}-\Delta \mathrm{mag}\right|>0.01$ and $\left|\Delta \mathrm{mag}_{1}-\Delta \mathrm{mag}\right|>0.01$ (Note that 0.01 is $< \pm 0.08 \%$ error on $\mathrm{dmag}$ ).

18. Remove duplicate solutions.

19. Verify that there are only two viable solutions.

20. Assign solutions to $\nu$.

\section{Appendix E: Projected Ellipse Semimajor Axis and Semiminor Axis, and $\theta$}

We write the analytical expressions for $a_{p}, b_{p}$, and $\theta$ using full expansions of Eqs. (13)-(15). These expressions are far too long to be practically conveyed, so we use the following intermediary parameters here and only here to simplify these expressions:

$$
\begin{aligned}
K_{0}= & e\left(1-e^{2}\right) \\
K_{1}= & \sin (\Omega) \cos (\omega)+\sin (\omega) \cos (\Omega) \cos (i) \\
K_{2}= & \sqrt{\frac{e+1}{1-e}} \\
K_{3}= & a\left(1-e^{2}\right) \\
K_{4}= & -\sin (\Omega) \sin (\omega) \cos (i)+\cos (\Omega) \cos (\omega) \\
K_{5}= & \sqrt{K_{1}^{2} K_{3}^{2}+K_{4}^{2} K_{3}^{2}+K_{3}^{2} \sin ^{2}(i) \sin ^{2}(\omega)} \\
K_{6}= & K_{3}(-\sin (\Omega) \cos (\omega)-\sin (\omega) \cos (\Omega) \cos (i)) /(1-e) \\
K_{7}= & K_{3}(\sin (\Omega) \sin (\omega) \cos (i)-\cos (\Omega) \cos (\omega)) /(1-e) \\
K_{8}= & K_{3}\left(\sin (\Omega) \cos \left(\omega+2 \tan ^{-1}\left(K_{2}\right)\right)+\sin (\omega\right. \\
& \left.\left.+2 \tan -1\left(K_{2}\right)\right) \cos (\Omega) \cos (i)\right) /\left(e \cos \left(2 \tan ^{-1}\left(K_{2}\right)\right)+1\right) \\
K_{9}= & K_{3}\left(-\sin (\Omega) \sin \left(\omega+2 \tan ^{-1}\left(K_{2}\right)\right) \cos (i)\right. \\
& \left.+\cos (\Omega) \cos \left(\omega+2 \tan ^{-1}\left(K_{2}\right)\right)\right) /\left(e \cos \left(2 \tan ^{-1}\left(K_{2}\right)\right)+1\right) .
\end{aligned}
$$

The simplified expression for the semimajor axis of the projected ellipse is

$$
\begin{aligned}
a_{p}= & \frac{1}{2} \sqrt{\left(\frac{K_{0} a^{2} K_{1}}{K_{5}}-\frac{K_{0} a^{2} K_{4}}{K_{5}}+K_{6}-K_{9}\right)^{2}+\left(\frac{K_{0} a^{2} K_{1}}{K_{5}}+\frac{K_{0} a^{2} K_{4}}{K_{5}}+K_{7}+K_{8}\right)^{2}} \\
& +\frac{1}{2} \sqrt{\left(\frac{K_{0} a^{2} K_{1}}{K_{5}}-\frac{K_{0} a^{2} K_{4}}{K_{5}}-K_{7}+K_{8}\right)^{2}+\left(\frac{K_{0} a^{2} K_{1}}{K_{5}}+\frac{K_{0} a^{2} K_{4}}{K_{5}}+K_{6}+K_{9}\right)^{2}} .
\end{aligned}
$$

The simplified expression for the projected ellipse semiminor axis is

$$
\begin{aligned}
b_{p}= & -\frac{1}{2} \sqrt{\left|\frac{K_{0} a^{2} K_{1}}{K_{5}}-\frac{K_{0} a^{2} K_{4}}{K_{5}}+K_{6}-K_{9}\right|^{2}+\left|\frac{K_{0} a^{2} K_{1}}{K_{5}}+\frac{K_{0} a^{2} K_{4}}{K_{5}}+K_{7}+K_{8}\right|^{2}} \\
& +\frac{1}{2} \sqrt{\left|\frac{K_{0} a^{2} K_{1}}{K_{5}}-\frac{K_{0} a^{2} K_{4}}{K_{5}}-K_{7}+K_{8}\right|^{2}+\left|\frac{K_{0} a^{2} K_{1}}{K_{5}}+\frac{K_{0} a^{2} K_{4}}{K_{5}}+K_{6}+K_{9}\right|^{2}} .
\end{aligned}
$$

The simplified expression for the angle between the projected ellipse semimajor axis and $X$ axis is

$$
\theta=\frac{1}{2}\left[\tan ^{-1}\left(\frac{\frac{K_{0} a^{2} K_{1}}{K_{5}}-\frac{K_{0} a^{2} K_{4}}{K_{5}}+K_{6}-K_{9}}{\frac{K_{0} a^{2} K_{1}}{K_{5}}+\frac{K_{0} a^{2} K_{4}}{K_{5}}+K_{7}+K_{8}}\right)+\tan ^{-1}\left(\frac{\frac{K_{0} a^{2} K_{1}}{K_{5}}+\frac{K_{0} a^{2} K_{4}}{K_{5}}+K_{6}+K_{9}}{-\frac{K_{0} a^{2} K_{1}}{K_{5}}+\frac{K_{0} a^{2} K_{4}}{K_{5}}+K_{7}-K_{8}}\right)\right] .
$$




\section{Appendix F: s Extrema Polynomial}

We reduced Eq. (22) from Sec. 2.1.2 into the standard reduced form of a quartic expression:

$$
\begin{aligned}
0= & x_{e}^{4}+\frac{-2 a_{p}^{2} x_{*}+2 b_{p}^{2} x_{*}}{\left(a_{p}^{4}-2 a_{p}^{2} b_{p}^{2}+b_{p}^{4}\right) / a_{p}^{2}} x_{e}^{3}+\frac{-a_{p}^{4}+2 a_{p}^{2} b_{p}^{2}+a_{p}^{2} x_{*}^{2}-b_{p}^{4}+b_{p}^{2} y_{*}^{2}}{\left(a_{p}^{4}-2 a_{p}^{2} b_{p}^{2}+b_{p}^{4}\right) / a_{p}^{2}} x_{e}^{2} \\
& +\frac{2 a_{p}^{4} x_{*}-2 a_{p}^{2} b_{p}^{2} x_{*}}{\left(a_{p}^{4}-2 a_{p}^{2} b_{p}^{2}+b_{p}^{4}\right) / a_{p}^{2}} x_{e}+\frac{-a_{p}^{4} x_{*}^{2}}{\left(a_{p}^{4}-2 a_{p}^{2} b_{p}^{2}+b_{p}^{4}\right) / a_{p}^{2}} .
\end{aligned}
$$

The coefficients of the quartic expression are

$$
\begin{gathered}
A_{0}=\frac{-2 a_{p}^{2} x_{*}+2 b_{p}^{2} x_{*}}{\left(a_{p}^{4}-2 a_{p}^{2} b_{p}^{2}+b_{p}^{4}\right) / a_{p}^{2}}, \\
B_{0}=\frac{-a_{p}^{4}+2 a_{p}^{2} b_{p}^{2}+a_{p}^{2} x_{*}^{2}-b_{p}^{4}+b_{p}^{2} y_{*}^{2}}{\left(a_{p}^{4}-2 a_{p}^{2} b_{p}^{2}+b_{p}^{4}\right) / a_{p}^{2}}, \\
C_{0}=\frac{2 a_{p}^{4} x_{*}-2 a_{p}^{2} b_{p}^{2} x_{*}}{\left(a_{p}^{4}-2 a_{p}^{2} b_{p}^{2}+b_{p}^{4}\right) / a_{p}^{2}}, \\
D_{0}=\frac{-a_{p}^{4} x_{*}^{2}}{\left(a_{p}^{4}-2 a_{p}^{2} b_{p}^{2}+b_{p}^{4}\right) / a_{p}^{2}} .
\end{gathered}
$$

\section{Appendix G: s Intersection Polynomial}

After dividing by the coefficient of $x_{e}^{4}$ in Eq. (35) from Sec. 2.1.3, we get

$$
\begin{aligned}
0= & x_{e}^{4}-\frac{4 a_{p}^{2} x_{*}}{a_{p}^{2}-b_{p}^{2}} x_{e}^{3}+\frac{2 a_{p}^{2}\left(a_{p}^{2} b_{p}^{2}-a_{p}^{2} s^{2}+3 a_{p}^{2} x_{*}^{2}+a_{p}^{2} y_{*}^{2}-b_{p}^{4}+b_{p}^{2} s^{2}-b_{p}^{2} x_{*}^{2}+b_{p}^{2} y_{*}^{2}\right)}{a_{p}^{4}-2 a_{p}^{2} b_{p}^{2}+b_{p}^{4}} x_{e}^{2} \\
& +\frac{4 a_{p}^{4} x_{*}\left(-b_{p}^{2}+s^{2}-x_{*}^{2}-y_{*}^{2}\right)}{a_{p}^{4}-2 a_{p}^{2} b_{p}^{2}+b_{p}^{4}} x_{e} \\
& +\frac{a_{p}^{4}\left(b_{p}^{4}-2 b_{p}^{2} s^{2}+2 b_{p}^{2} x_{*}^{2}-2 b_{p}^{2} y_{*}^{2}+s^{4}-2 s^{2} x_{*}^{2}-2 s^{2} y_{*}^{2}+x_{*}^{4}+2 x_{*}^{2} y_{*}^{2}+y_{*}^{4}\right)}{a_{p}^{4}-2 a_{p}^{2} b_{p}^{2}+b_{p}^{4}}
\end{aligned}
$$

The coefficients of the general quartic expression in Eq. (36) are

$$
\begin{gathered}
A_{1}=-\frac{4 a_{p}^{2} x_{*}}{a_{p}^{2}-b_{p}^{2}} \\
B_{1}=\frac{2 a_{p}^{2}\left(a_{p}^{2} b_{p}^{2}-a_{p}^{2} s^{2}+3 a_{p}^{2} x_{*}^{2}+a_{p}^{2} y_{*}^{2}-b_{p}^{4}+b_{p}^{2} s^{2}-b_{p}^{2} x_{*}^{2}+b_{p}^{2} y_{*}^{2}\right)}{a_{p}^{4}-2 a_{p}^{2} b_{p}^{2}+b_{p}^{4}}, \\
C_{1}=\frac{4 a_{p}^{4} x_{*}\left(-b_{p}^{2}+s^{2}-x_{*}^{2}-y_{*}^{2}\right)}{a_{p}^{4}-2 a_{p}^{2} b_{p}^{2}+b_{p}^{4}}
\end{gathered}
$$

and

$$
D_{1}=\frac{a_{p}^{4}\left(b_{p}^{4}-2 b_{p}^{2} s^{2}+2 b_{p}^{2} x_{*}^{2}-2 b_{p}^{2} y_{*}^{2}+s^{4}-2 s^{2} x_{*}^{2}-2 s^{2} y_{*}^{2}+x_{*}^{4}+2 x_{*}^{2} y_{*}^{2}+y_{*}^{4}\right)}{a_{p}^{4}-2 a_{p}^{2} b_{p}^{2}+b_{p}^{4}}
$$




\section{Appendix H: $\Delta$ mag Extrema Polynomial}

Here we include the coefficients of Eq. (49) from Sec. 2.2 as

$$
\begin{aligned}
& A_{2}=e^{4} \sin ^{4}(i), \\
& B_{2}=3 e^{3}(e \sin (\omega)+\sin (i)) \sin ^{3}(i), \\
& C_{2}=\frac{1}{4} e^{2}\left(4 e^{2} \sin ^{2}(i) \sin ^{2}(\omega)-8 e^{2} \sin ^{2}(i)+8 e^{2} \sin ^{2}(\omega)+5 e^{2}+34 e \sin (i) \sin (\omega)\right. \\
& \left.+13 \sin ^{2}(i)\right) \sin ^{2}(i), \\
& D_{2}=\frac{1}{2} e\left[2 e^{3} \sin ^{2}(i) \sin ^{3}(\omega)-8 e^{3} \sin ^{2}(i) \sin (\omega)+3 e^{3} \sin (\omega)+5 e^{2} \sin ^{3}(i) \sin ^{2}(\omega)\right. \\
& -11 e^{2} \sin ^{3}(i)+10 e^{2} \sin (i) \sin ^{2}(\omega)+7 e^{2} \sin (i)+17 e \sin ^{2}(i) \sin (\omega) \\
& \left.+3 \sin ^{3}(i)\right) \sin (i), \\
& E_{2}=-\frac{5 e^{4} \sin ^{4}(i) \sin ^{2}(\omega)}{4}+\frac{5 e^{4} \sin ^{4}(i)}{4}-\frac{7 e^{4} \sin ^{2}(i) \sin ^{2}(\omega)}{4}-\frac{3 e^{4} \sin ^{2}(i)}{2} \\
& +\frac{e^{4}}{4}+\frac{3 e^{3} \sin ^{3}(i) \sin ^{3}(\omega)}{2}-10 e^{3} \sin ^{3}(i) \sin (\omega)+\frac{7 e^{3} \sin (i) \sin (\omega)}{2} \\
& +2 e^{2} \sin ^{4}(i) \sin ^{2}(\omega)-\frac{21 e^{2} \sin ^{4}(i)}{4}+4 e^{2} \sin ^{2}(i) \sin ^{2}(\omega)+\frac{7 e^{2} \sin ^{2}(i)}{2} \\
& +\frac{7 e \sin ^{3}(i) \sin (\omega)}{2}+\frac{\sin ^{4}(i)}{4}, \\
& F_{2}=-\frac{3 e^{4} \sin ^{3}(i) \sin ^{3}(\omega)}{2}+\frac{3 e^{4} \sin ^{3}(i) \sin (\omega)}{2}-\frac{3 e^{4} \sin (i) \sin (\omega)}{2}-\frac{e^{3} \sin ^{4}(i) \sin ^{4}(\omega)}{2} \\
& -\frac{5 e^{3} \sin ^{4}(i) \sin ^{2}(\omega)}{2}+3 e^{3} \sin ^{4}(i)-5 e^{3} \sin ^{2}(i) \sin ^{2}(\omega)-\frac{7 e^{3} \sin ^{2}(i)}{2}+\frac{e^{3}}{2} \\
& -\frac{e^{2} \sin ^{3}(i) \sin ^{3}(\omega)}{2}-8 e^{2} \sin ^{3}(i) \sin (\omega)+\frac{5 e^{2} \sin (i) \sin (\omega)}{2}+\frac{e \sin ^{4}(i) \sin ^{2}(\omega)}{2} \\
& -2 e \sin ^{4}(i)+e \sin ^{2}(i) \sin ^{2}(\omega)+\frac{3 e \sin ^{2}(i)}{2}+\frac{\sin ^{3}(i) \sin (\omega)}{2}, \\
& G_{2}=-\frac{e^{4} \sin ^{4}(i) \sin ^{4}(\omega)}{4}+\frac{e^{4} \sin ^{4}(i) \sin ^{2}(\omega)}{2}-\frac{e^{4} \sin ^{4}(i)}{4}-\frac{e^{4} \sin ^{2}(i) \sin ^{2}(\omega)}{2} \\
& +\frac{e^{4} \sin ^{2}(i)}{2}-\frac{e^{4}}{4}-\frac{7 e^{3} \sin ^{3}(i) \sin ^{3}(\omega)}{2}+\frac{7 e^{3} \sin ^{3}(i) \sin (\omega)}{2}-\frac{7 e^{3} \sin (i) \sin (\omega)}{2} \\
& -\frac{5 e^{2} \sin ^{4}(i) \sin ^{4}(\omega)}{4}-e^{2} \sin ^{4}(i) \sin ^{2}(\omega)+\frac{9 e^{2} \sin ^{4}(i)}{4}-5 e^{2} \sin ^{2}(i) \sin ^{2}(\omega) \\
& -\frac{5 e^{2} \sin ^{2}(i)}{2}+\frac{e^{2}}{4}-\frac{3 e \sin ^{3}(i) \sin ^{3}(\omega)}{2}-2 e \sin ^{3}(i) \sin (\omega)+\frac{e \sin (i) \sin (\omega)}{2} \\
& -\frac{\sin ^{4}(i)}{4}+\frac{\sin ^{2}(i)}{4} \\
& H_{2}=-\frac{e^{3} \sin ^{4}(i) \cos ^{4}(\omega)}{2}+e^{3} \sin ^{2}(i) \cos ^{2}(\omega)-\frac{e^{3}}{2}+\frac{5 e^{2} \sin ^{3}(i) \sin (\omega) \cos ^{2}(\omega)}{2} \\
& -\frac{5 e^{2} \sin (i) \sin (\omega)}{2}-\frac{3 e(\cos 2(i)-1)^{2}(\cos (4 w)-1)}{64}+\frac{e \sin ^{4}(i) \cos ^{4}(\omega)}{2} \\
& -\frac{5 e \sin ^{2}(i) \sin ^{2}(\omega)}{2}-\frac{e \sin ^{2}(i) \cos ^{2}(\omega)}{2}-\frac{\sin ^{3}(i) \sin ^{3}(\omega)}{2}, \quad \text { and } \\
& I_{2}=-\frac{e^{2} \sin ^{4}(i) \cos ^{4}(\omega)}{4}+\frac{e^{2} \sin ^{2}(i) \cos ^{2}(\omega)}{2}-\frac{e^{2}}{4}+\frac{e \sin ^{3}(i) \sin (\omega) \cos ^{2}(\omega)}{2} \\
& -\frac{e \sin (i) \sin (\omega)}{2}-\frac{(\cos (2 i)-1)^{2}(\cos (4 w)-1)}{128}-\frac{\sin ^{2}(i) \sin ^{2}(\omega)}{4} .
\end{aligned}
$$




\section{Appendix I: $\Delta$ mag Intersection Polynomial}

Here we include the coefficients of Eq. (50) from Sec. 2.2 as

$$
\begin{aligned}
& A_{3}=\frac{e^{4} \sin ^{4}(i)}{16}, \\
& B_{3}=\frac{1}{4} e^{3}(e \sin (\omega)+\sin (i)) \sin ^{3}(i), \\
& C_{3}=\frac{1}{8} e^{2}\left(e^{2} \cos ^{2}(i) \cos ^{2}(\omega)-3 e^{2} \cos ^{2}(\omega)+3 e^{2}+8 e \sin (i) \sin (\omega)-3 \cos ^{2}(i)\right. \\
& +3) \sin ^{2}(i) \text {, } \\
& D_{3}=\frac{1}{4} e\left[-e^{3} \sin ^{3}(\omega) \cos ^{2}(i)+e^{3} \sin ^{3}(\omega)+e^{3} \sin (\omega) \cos ^{2}(i)-2 e^{2} \sin ^{3}(i) \cos ^{2}(\omega)\right. \\
& -4 e^{2} \sin (i) \cos ^{2}(\omega)+6 e^{2} \sin (i)-6 e \sin (\omega) \cos ^{2}(i)+6 e \sin (\omega) \\
& \left.+\sin ^{3}(i)\right) \sin (i), \\
& E_{3}=\frac{e^{4} \sin ^{4}(i) \sin ^{4}(\omega)}{16}-\frac{e^{4} \sin ^{4}(i) \sin ^{2}(\omega)}{8}+\frac{e^{4} \sin ^{4}(i)}{16}+\frac{e^{4} \sin ^{2}(i) \sin ^{2}(\omega)}{8} \\
& -\frac{e^{4} \sin ^{2}(i)}{8}+\frac{e^{4}}{16}+e^{3} \sin ^{3}(i) \sin ^{3}(\omega)-e^{3} \sin ^{3}(i) \sin (\omega)+e^{3} \sin (i) \sin (\omega) \\
& -e^{2} \xi \sin ^{2}(i) \sin ^{2}(\omega)+\frac{e^{2} \xi \sin ^{2}(i)}{2}+\frac{3 e^{2} \sin ^{4}(i) \sin ^{2}(\omega)}{4}-\frac{3 e^{2} \sin ^{4}(i)}{4} \\
& +\frac{3 e^{2} \sin ^{2}(i) \sin ^{2}(\omega)}{2}+\frac{3 e^{2} \sin ^{2}(i)}{4}+e \sin ^{3}(i) \sin (\omega)+\frac{\sin ^{4}(i)}{16}, \\
& F_{3}=\frac{e^{3} \sin ^{4}(i) \sin ^{4}(\omega)}{4}-\frac{e^{3} \sin ^{4}(i) \sin ^{2}(\omega)}{2}+\frac{e^{3} \sin ^{4}(i)}{4}+\frac{e^{3} \sin ^{2}(i) \sin ^{2}(\omega)}{2} \\
& -\frac{e^{3} \sin ^{2}(i)}{2}+\frac{e^{3}}{4}-e^{2} \xi \sin (i) \sin (\omega)+\frac{3 e^{2} \sin ^{3}(i) \sin ^{3}(\omega)}{2} \\
& -\frac{3 e^{2} \sin ^{3}(i) \sin (\omega)}{2}+\frac{3 e^{2} \sin (i) \sin (\omega)}{2}-2 e \xi \sin ^{2}(i) \sin ^{2}(\omega)+e \xi \sin ^{2}(i) \\
& +\frac{e \sin ^{4}(i) \sin ^{2}(\omega)}{2}-\frac{e \sin ^{4}(i)}{2}+e \sin ^{2}(i) \sin ^{2}(\omega)+\frac{e \sin ^{2}(i)}{2} \\
& +\frac{\sin ^{3}(i) \sin (\omega)}{4}, \\
& G_{3}=-\frac{e^{2} \xi \sin ^{2}(i) \cos ^{2}(\omega)}{2}-\frac{e^{2} \xi}{2}+\frac{3 e^{2} \sin ^{4}(i) \cos ^{4}(\omega)}{8}-\frac{3 e^{2} \sin ^{2}(i) \cos ^{2}(\omega)}{4} \\
& +\frac{3 e^{2}}{8}-2 e \xi \sin (i) \sin (\omega)-e \sin ^{3}(i) \sin (\omega) \cos ^{2}(\omega)+e \sin (i) \sin (\omega) \\
& -\frac{\xi \sin ^{2}(i) \sin ^{2}(\omega)}{2}+\frac{\xi \sin ^{2}(i) \cos ^{2}(\omega)}{2}+\frac{(\cos (2 i)-1)^{2}(\cos (4 w)-1)}{256} \\
& -\frac{\sin ^{4}(i) \cos ^{4}(\omega)}{8}+\frac{3 \sin ^{2}(i) \sin ^{2}(\omega)}{8}+\frac{\sin ^{2}(i) \cos ^{2}(\omega)}{8}, \\
& H_{3}=-e \xi \sin ^{2}(i) \cos ^{2}(\omega)-e \xi+\frac{e \sin ^{4}(i) \cos ^{4}(\omega)}{4}-\frac{e \sin ^{2}(i) \cos ^{2}(\omega)}{2}+\frac{e}{4} \\
& -\xi \sin (i) \sin (\omega)-\frac{\sin ^{3}(i) \sin (\omega) \cos ^{2}(\omega)}{4}+\frac{\sin (i) \sin (\omega)}{4}, \quad \text { and } \\
& I_{3}=\xi^{2}-\frac{\xi \sin ^{2}(i) \cos ^{2}(\omega)}{2}-\frac{\xi}{2}+\frac{\sin ^{4}(i) \cos ^{4}(\omega)}{16}-\frac{\sin ^{2}(i) \cos ^{2}(\omega)}{8}+\frac{1}{16} \text {. }
\end{aligned}
$$




\section{Appendix J: Proof that Ellipses Project to Ellipses}

Let us suppose that we have some ellipse on an arbitrary plane indicated by the black ellipse in Fig. 4. We will say $\overline{A B}$ and $\overline{C D}$ are the principal axes of some this $3 \mathrm{D}$ ellipse. $\overline{A B}$ and $\overline{C D}$ intersect at point $O$, the geometric center of this 3D ellipse. We will say point $P$ is any point on the ellipse with $H$ being the projection of point $P$ onto axis $\overline{A B}$ and $K$ being the projection of point $P$ onto axis $\overline{C D}$. By the definition of an ellipse, we have

$$
\frac{\overline{O H}^{2}}{\overline{O B}^{2}}+\frac{\overline{O K}^{2}}{\overline{O D}^{2}}=1 .
$$

Now we let $A^{\prime}, B^{\prime}, C^{\prime}, D^{\prime}, O^{\prime}, K^{\prime}, H^{\prime}$, and $P^{\prime}$ be the perpendicular projections of points $A$, $B, C, D, O, K, H$, and $P$ onto any given plane. Since perpendicular projections preserve the ratios of segments on a line, we can now say

$$
\frac{\overline{O^{\prime} H^{\prime}}}{\overline{O^{\prime} B^{\prime 2}}}+\frac{\overline{O^{\prime} K^{\prime}}}{\overline{O^{\prime} D^{\prime 2}}}=1 .
$$

This equation means that point $P^{\prime}$ belongs to the ellipse having $A^{\prime} B^{\prime}$ and $C^{\prime} D^{\prime}$ as conjugate diameters. For an ellipse, two diameters are conjugate if and only if the tangent line to the ellipse at an endpoint of one diameter is parallel to the other diameter.

\section{Appendix K: Quartic Solution}

The quartic function has a known analytical solution. ${ }^{19}$ We start with a set of useful simplifying terms

$$
\begin{aligned}
& p_{0}=\left(-\frac{3 A^{2}}{8}+B\right)^{3} \text {, } \\
& p_{1}=\left(A\left(\frac{A^{2}}{8}-\frac{B}{2}\right)+C\right)^{2} \text {, } \\
& p_{2}=-A\left(A\left(\frac{3 A^{2}}{256}-\frac{B}{16}\right)+\frac{C}{4}\right)+D \text {, } \\
& p_{3}=-\frac{3 A^{2}}{8}+B \\
& p_{4}=2 A\left(\frac{A^{2}}{8}-\frac{B}{2}\right), \\
& p_{5}=-\frac{p_{0}}{108}-\frac{p_{1}}{8}+\frac{p_{2} p_{3}}{3}, \\
& p_{6}=\sqrt[3]{\frac{p_{0}}{216}+\frac{p_{1}}{16}-\frac{p_{2} p_{3}}{6}+\sqrt{\frac{p_{5}^{2}}{4}+\frac{\left(-p_{2}-\frac{p_{3}^{2}}{12}\right)^{3}}{27}}}, \\
& p_{7}=\frac{A^{2}}{4}-\frac{2 B}{3},
\end{aligned}
$$




$$
\begin{gathered}
p_{8}=\frac{2 p_{2}+\frac{p_{3}^{2}}{6}}{3 p_{6}}, \\
p_{9}=\sqrt{-2 \sqrt[3]{p_{5}}+p_{7}}, \\
p_{10}=\sqrt{2 p_{6}+p_{7}+p_{8}},
\end{gathered}
$$

and

$$
p_{11}=\frac{A^{2}}{2}-\frac{4 B}{3}
$$

The solutions are four piecewise functions:

$$
\begin{aligned}
& x_{0}=\left\{\begin{array}{ll}
-\frac{A}{4}+\frac{p_{9}}{2}-\frac{\sqrt{p_{11}+2 \sqrt[3]{p_{5}}+\frac{-2 C-p_{4}}{p_{9}}}}{2} & \text { for } p_{2}+\frac{p_{3}^{2}}{12}=0 \\
-\frac{A}{4}-\frac{p_{10}}{2}-\frac{\sqrt{p_{11}-2 p_{6}+p_{9}+\frac{2 C+p_{4}}{p_{10}}}}{2} & \text { otherwise }
\end{array},\right. \\
& x_{1}=\left\{\begin{array}{ll}
-\frac{A}{4}+\frac{p_{9}}{2}+\frac{\sqrt{p_{11}+2 \sqrt[3]{p_{5}}-\frac{2 C+p_{4}}{p_{9}}}}{2} & \text { for } p_{2}+\frac{p_{3}^{2}}{12}=0 \\
-\frac{A}{4}-\frac{p_{10}}{2}+\frac{\sqrt{p_{11}-2 p_{6}+p_{9}+\frac{2 C+p_{4}}{p_{10}}}}{2} & \text { otherwise }
\end{array},\right. \\
& x_{2}= \begin{cases}-\frac{A}{4}-\frac{p_{9}}{2}-\frac{\sqrt{p_{11}+2 \sqrt[3]{p_{5}}-\frac{-2 C-p_{4}}{p_{9}}}}{2} & \text { for } p_{2}+\frac{p_{3}^{2}}{12}=0 \\
-\frac{A}{4}+\frac{p_{10}}{2}-\frac{\sqrt{p_{11}-2 p_{6}+p_{9}+\frac{-2 C-p_{4}}{p_{10}}}}{2} & \text { otherwise }\end{cases}
\end{aligned}
$$

and

$$
x_{3}= \begin{cases}-\frac{A}{4}-\frac{p_{9}}{2}+\frac{\sqrt{p_{11}+2 \sqrt[3]{p_{5}}-\frac{-2 C-p_{4}}{p_{9}}}}{2} & \text { for } p_{2}+\frac{p_{3}^{2}}{12}=0 \\ -\frac{A}{4}+\frac{p_{10}}{2}+\frac{\sqrt{p_{11}-2 p_{6}+p_{9}+\frac{-2 C-p_{4}}{p_{10}}}}{2} & \text { otherwise }\end{cases}
$$

Theoretically, we always have four solutions. Theoretically, we can use equations of coefficients to determine how many roots are real, complex, and double roots. The general form of the quartic defines the expressions $\Delta, P, D_{2}, R$, and $\Delta_{0}$, which are

$$
\begin{aligned}
\Delta= & 256 D^{3}-192 A C D^{2}-128 B^{2} D^{2}+144 B C^{2} D-27 C^{4}+144 A^{2} B D^{2}-6 A^{2} C^{2} D \\
& -80 A B^{2} C D+18 A B C^{3}+16 B^{4} D-4 B^{3} C^{2}-27 A^{4} D^{2}+18 A^{3} B C D-4 A^{3} C^{3} \\
& -4 A^{2} B^{3} D+A^{2} B^{2} C^{2},
\end{aligned}
$$

$$
P=8 B-3 A^{2},
$$

$$
D_{2}=64 D-16 B^{2}+16 A^{2} B-16 A C-3 A^{4},
$$




$$
\begin{gathered}
R=A^{3}+8 C-4 A B, \\
\Delta_{0}=B^{2}-3 A C+12 D .
\end{gathered}
$$

After evaluation of these constants, we can determine

$$
\text { solution types }= \begin{cases}2 \text { distinct } \Re \text { roots \& } 2 \text { complex conjugate roots } & \Delta<0 \\ 4 \Re \text { distinct roots } & \Delta>0 \& P<0 \& D<0 \\ 2 \text { pair complex conjugate roots } & \Delta>0 \&(P>0 \mid D>0)\end{cases}
$$

However, the use of this theoretical classification does not work when numerical errors are introduced. The accumulation of numerical errors causes the solutions to be improperly classified for a sufficiently large number of cases to prohibit the use of these classifications. The $\Delta$ expression rarely evaluates to $\Delta=0$ when it should do so quite frequently. Numerical rounding errors can also frequently result in the evaluation $\Delta<0$ when in reality $\Delta>0$ and vice versa.

\section{Appendix L: Earth-Like Subpopulation}

We use a definition of Earth-like exoplanet similar to that used in Ref. 8. We use a more conservative planetary radius range of $0.9 R_{\oplus} \leq R \leq 1.4 R_{\oplus}$. We use the flux-at-planet range of $0.3586 \leq L_{\mathrm{plan}} \leq 1.1080$. To classify a planet, we use the time-averaged incident flux on the planet calculated by

$$
L_{\mathrm{plan}}=\frac{L_{*}}{\left(a+\frac{a e^{2}}{2}\right)^{2}}
$$

\section{Appendix M: 4-m Monolith Design Reference Mission}

We calculated the Brown completeness and integration time adjusted completeness for a 4-m monolith telescope with a contrast of $10^{-10}$ on the SAG13 planet population discussed in Ref. 1. The $\Delta$ mag and separations of integration are scaled by the luminosity of each candidate target star. The orbital periods are similarly scaled by the mass of each target star in the case of integration time adjusted completeness. We optimized the mission schedule using the sequential least squares quadratic programming method discussed in Ref. 1 with the Brown completeness method. Our approach included the adoption of the use of the local zodiacal light minimum and filtering of target stars.

We assume a total telescope time of 213.67 days ( $\sim 7$ months) and that each observation requires 0.1 day of overhead time and 0.042 day settling time. The coronagraph parameters used are from a vortex charge six coronagraph at a wavelength of $500 \mathrm{~nm}$ with an IWA of 0.045 arc sec and OWA of 2.127 arc sec and a working angle of integration consistent with that used in Ref. 1.

The resulting Brown completeness optimized integration times for a DRM are included in Table 2. The summed Brown completeness for this DRM is 68.89 , whereas the same summed integration time adjusted completeness is 63.11 , a difference of 5.78 . We can multiply either of these numbers by the SAG13 exoplanet occurrence rate of 5.62 to get the expected number of exoplanets detected in a single-visit blind search. These are 387.16 and 354.67 on average, respectively. This means that 32.49 planets are lost in a mission by not taking into account integration times when optimizing the mission schedule. 
Table 2 4-m monolith DRM.

\begin{tabular}{|c|c|c|c|c|c|}
\hline Name & Dist (pc) & Int. time (day) & $\Delta \mathrm{mag}$ & $C_{\text {brown }}$ & $C_{\mathrm{IAC}}$ \\
\hline HIP 439 & 4.34 & 0.399 & 19.557 & 0.049 & 0.041 \\
\hline HIP 522 & 25.71 & 0.181 & 23.476 & 0.038 & 0.033 \\
\hline HIP 544 & 13.67 & 0.6 & 23.403 & 0.16 & 0.141 \\
\hline HIP 746 & 16.78 & 0.066 & 27.142 & 0.247 & 0.24 \\
\hline HIP 910 & 18.75 & 0.383 & 24.916 & 0.16 & 0.146 \\
\hline HIP 950 & 21.28 & 0.341 & 24.504 & 0.106 & 0.095 \\
\hline HIP 1292 & 17.5 & 0.338 & 22.181 & 0.054 & 0.047 \\
\hline HIP 1392 B & 15.19 & 0.293 & 21.362 & 0.039 & 0.033 \\
\hline HIP 1475 & 3.57 & 0.625 & 20.237 & 0.105 & 0.089 \\
\hline HIP 1499 & 23.25 & 0.139 & 21.736 & 0.018 & 0.016 \\
\hline HIP 1599 & 8.59 & 0.374 & 25.793 & 0.47 & 0.438 \\
\hline HIP 1803 & 20.86 & 0.241 & 22.209 & 0.036 & 0.031 \\
\hline HIP 2021 & 7.46 & 0.179 & 26.942 & 0.55 & 0.525 \\
\hline HIP 2072 & 23.81 & 0.129 & 25.773 & 0.103 & 0.097 \\
\hline HIP 2081 & 25.97 & 0.053 & 26.432 & 0.082 & 0.078 \\
\hline HIP 2711 & 25.48 & 0.193 & 23.752 & 0.045 & 0.039 \\
\hline HIP 3093 & 11.06 & 0.745 & 23.731 & 0.242 & 0.213 \\
\hline HIP 3170 & 24.96 & 0.192 & 23.257 & 0.038 & 0.033 \\
\hline HIP 3419 & 29.53 & 0.035 & 26.545 & 0.048 & 0.046 \\
\hline HIP 3497 & 22.06 & 0.184 & 21.928 & 0.025 & 0.022 \\
\hline HIP 3505 & 26.75 & 0.169 & 24.171 & 0.044 & 0.039 \\
\hline HIP 3583 & 15.16 & 0.567 & 23.919 & 0.17 & 0.151 \\
\hline HIP 3765 & 7.45 & 0.861 & 23.974 & 0.38 & 0.339 \\
\hline HIP 3810 & 23.45 & 0.27 & 24.446 & 0.078 & 0.07 \\
\hline HIP 3909 & 15.75 & 0.515 & 24.685 & 0.207 & 0.187 \\
\hline HIP 4148 & 14.17 & 0.293 & 21.192 & 0.039 & 0.033 \\
\hline HIP 4151 & 18.74 & 0.353 & 25.07 & 0.166 & 0.152 \\
\hline HIP 5336 & 7.55 & 0.66 & 24.859 & 0.459 & 0.418 \\
\hline HIP 5862 & 15.11 & 0.46 & 25.001 & 0.238 & 0.217 \\
\hline HIP 6379 & 16.81 & 0.217 & 21.188 & 0.028 & 0.024 \\
\hline HIP 6706 & 25.21 & 0.207 & 23.99 & 0.051 & 0.046 \\
\hline HIP 6813 & 28.62 & 0.13 & 24.535 & 0.037 & 0.033 \\
\hline HIP 7235 & 19.05 & 0.164 & 21.17 & 0.021 & 0.018 \\
\hline HIP 7339 & 20.66 & 0.236 & 22.106 & 0.035 & 0.03 \\
\hline
\end{tabular}


Table 2 (Continued).

\begin{tabular}{|c|c|c|c|c|c|}
\hline Name & Dist $(p c)$ & Int. time (day) & $\Delta$ mag & $C_{\text {brown }}$ & $C_{\mathrm{IAC}}$ \\
\hline HIP 7513 & 13.49 & 0.296 & 25.765 & 0.309 & 0.288 \\
\hline HIP 7601 & 27.38 & 0.129 & 23.04 & 0.023 & 0.02 \\
\hline HIP 7734 & 21.37 & 0.17 & 21.643 & 0.022 & 0.019 \\
\hline HIP 7918 & 12.74 & 0.521 & 24.978 & 0.295 & 0.269 \\
\hline HIP 7978 & 17.43 & 0.483 & 24.302 & 0.153 & 0.137 \\
\hline HIP 7981 & 7.53 & 0.733 & 24.611 & 0.44 & 0.398 \\
\hline HIP 8102 & 3.65 & 0.323 & 26.364 & 0.745 & 0.704 \\
\hline HIP 8362 & 10.07 & 0.753 & 24.254 & 0.318 & 0.285 \\
\hline HIP 8433 & 27.86 & 0.119 & 23.024 & 0.021 & 0.019 \\
\hline HIP 8497 & 23.19 & 0.241 & 25.078 & 0.098 & 0.09 \\
\hline HIP 8796 & 19.42 & 0.141 & 26.116 & 0.18 & 0.17 \\
\hline HIP 8903 & 17.99 & 0.08 & 26.814 & 0.217 & 0.209 \\
\hline HIP 9007 & 17.85 & 0.199 & 25.89 & 0.207 & 0.194 \\
\hline HIP 9236 & 22.01 & 0.069 & 26.565 & 0.138 & 0.132 \\
\hline HIP 9884 & 20.18 & 0.069 & 26.753 & 0.172 & 0.165 \\
\hline HIP 10138 & 10.78 & 0.746 & 23.544 & 0.231 & 0.204 \\
\hline HIP 10306 & 28.87 & 0.115 & 23.779 & 0.026 & 0.023 \\
\hline HIP 10644 & 10.78 & 0.528 & 25.092 & 0.359 & 0.329 \\
\hline HIP 10723 & 24.35 & 0.218 & 23.513 & 0.047 & 0.041 \\
\hline HIP 10798 & 12.67 & 0.605 & 22.975 & 0.146 & 0.128 \\
\hline HIP 11029 & 29.66 & 0.097 & 23.551 & 0.02 & 0.018 \\
\hline HIP 11783 & 26.7 & 0.179 & 24.768 & 0.055 & 0.049 \\
\hline HIP 12114 & 7.18 & 0.88 & 23.866 & 0.379 & 0.337 \\
\hline HIP 12186 & 25.78 & 0.173 & 23.307 & 0.034 & 0.03 \\
\hline HIP 12444 & 21.76 & 0.299 & 23.526 & 0.067 & 0.059 \\
\hline HIP 12623 & 24.19 & 0.249 & 24.661 & 0.076 & 0.068 \\
\hline HIP 12653 & 17.17 & 0.488 & 24.473 & 0.167 & 0.15 \\
\hline HIP 12777 & 11.13 & 0.324 & 25.829 & 0.381 & 0.356 \\
\hline HIP 12843 & 14.22 & 0.352 & 25.471 & 0.28 & 0.259 \\
\hline HIP 13402 & 10.35 & 0.771 & 23.572 & 0.245 & 0.216 \\
\hline HIP 13665 & 26.71 & 0.159 & 23.616 & 0.035 & 0.031 \\
\hline HIP 14146 & 27.17 & 0.112 & 25.539 & 0.062 & 0.058 \\
\hline HIP 14150 & 20.64 & 0.162 & 21.435 & 0.021 & 0.018 \\
\hline HIP 14632 & 10.54 & 0.328 & 25.855 & 0.402 & 0.375 \\
\hline
\end{tabular}


Table 2 (Continued).

\begin{tabular}{|c|c|c|c|c|c|}
\hline Name & Dist (pc) & Int. time (day) & $\Delta \mathrm{mag}$ & $C_{\text {brown }}$ & $C_{\mathrm{IAC}}$ \\
\hline HIP 14954 & 22.58 & 0.299 & 24.511 & 0.09 & 0.08 \\
\hline HIP 15330 & 12.01 & 0.669 & 24.437 & 0.278 & 0.25 \\
\hline HIP 15371 & 12.03 & 0.592 & 24.779 & 0.303 & 0.275 \\
\hline HIP 15457 & 9.14 & 0.567 & 25.073 & 0.413 & 0.378 \\
\hline HIP 15510 & 6.04 & 0.426 & 25.751 & 0.581 & 0.541 \\
\hline HIP 16245 & 21.68 & 0.264 & 25.16 & 0.121 & 0.111 \\
\hline HIP 16537 & 3.21 & 0.377 & 26.121 & 0.768 & 0.722 \\
\hline HIP 16852 & 13.96 & 0.339 & 25.542 & 0.289 & 0.268 \\
\hline HIP 17378 & 9.04 & 0.286 & 26.162 & 0.466 & 0.438 \\
\hline HIP 17420 & 13.95 & 0.326 & 21.324 & 0.044 & 0.038 \\
\hline HIP 17439 & 16.03 & 0.286 & 21.475 & 0.039 & 0.033 \\
\hline HIP 17651 & 17.63 & 0.25 & 25.64 & 0.206 & 0.192 \\
\hline HIP 18859 & 18.83 & 0.429 & 24.311 & 0.132 & 0.118 \\
\hline HIP 19076 & 16.94 & 0.466 & 23.433 & 0.114 & 0.101 \\
\hline HIP 19205 & 27.6 & 0.147 & 24.021 & 0.036 & 0.032 \\
\hline HIP 19335 & 21.0 & 0.341 & 23.973 & 0.09 & 0.08 \\
\hline HIP 19849 & 4.98 & 0.504 & 25.516 & 0.625 & 0.579 \\
\hline HIP 19859 & 21.33 & 0.244 & 22.41 & 0.039 & 0.034 \\
\hline HIP 19884 & 13.04 & 0.19 & 20.397 & 0.024 & 0.02 \\
\hline HIP 19893 & 20.46 & 0.206 & 25.608 & 0.152 & 0.141 \\
\hline HIP 19921 & 18.24 & 0.322 & 25.261 & 0.183 & 0.168 \\
\hline HIP 19990 & 28.94 & 0.122 & 24.383 & 0.033 & 0.03 \\
\hline HIP 21421 & 20.43 & 0.039 & 27.351 & 0.17 & 0.166 \\
\hline HIP 21547 & 29.43 & 0.107 & 23.929 & 0.025 & 0.022 \\
\hline HIP 21770 & 20.17 & 0.24 & 25.449 & 0.153 & 0.141 \\
\hline HIP 21861 & 28.67 & 0.127 & 24.318 & 0.034 & 0.03 \\
\hline HIP 22263 & 13.28 & 0.635 & 24.402 & 0.243 & 0.218 \\
\hline HIP 22336 & 26.42 & 0.154 & 23.183 & 0.029 & 0.026 \\
\hline HIP 22449 & 8.07 & 0.218 & 26.617 & 0.516 & 0.491 \\
\hline HIP 23311 & 8.71 & 0.832 & 23.26 & 0.258 & 0.226 \\
\hline HIP 23482 & 26.08 & 0.185 & 24.074 & 0.047 & 0.042 \\
\hline HIP 23693 & 11.65 & 0.434 & 25.351 & 0.346 & 0.319 \\
\hline HIP 23783 & 26.29 & 0.189 & 24.587 & 0.055 & 0.049 \\
\hline HIP 23875 & 27.4 & 0.044 & 26.48 & 0.067 & 0.064 \\
\hline
\end{tabular}


Table 2 (Continued).

\begin{tabular}{|c|c|c|c|c|c|}
\hline Name & Dist $(p c)$ & Int. time (day) & $\Delta$ mag & $C_{\text {brown }}$ & $C_{\mathrm{IAC}}$ \\
\hline HIP 23941 & 25.46 & 0.208 & 24.364 & 0.057 & 0.051 \\
\hline HIP 24186 & 3.91 & 0.377 & 19.298 & 0.044 & 0.037 \\
\hline HIP 24786 & 25.03 & 0.183 & 23.045 & 0.034 & 0.029 \\
\hline HIP 24813 & 12.63 & 0.458 & 25.207 & 0.311 & 0.285 \\
\hline HIP 25110 & 20.89 & 0.343 & 24.749 & 0.12 & 0.108 \\
\hline HIP 25278 & 14.39 & 0.517 & 24.847 & 0.246 & 0.224 \\
\hline HIP 25544 & 19.2 & 0.183 & 21.347 & 0.024 & 0.02 \\
\hline HIP 25623 & 13.02 & 0.163 & 20.148 & 0.02 & 0.017 \\
\hline HIP 25878 & 5.68 & 0.483 & 20.34 & 0.068 & 0.058 \\
\hline HIP 26394 & 18.32 & 0.446 & 24.074 & 0.128 & 0.114 \\
\hline HIP 26779 & 12.28 & 0.645 & 23.215 & 0.171 & 0.15 \\
\hline HIP 27072 & 8.93 & 0.256 & 26.326 & 0.474 & 0.448 \\
\hline HIP 27288 & 21.61 & 0.108 & 26.165 & 0.141 & 0.134 \\
\hline HIP 27321 & 19.44 & 0.155 & 26.012 & 0.178 & 0.167 \\
\hline HIP 27435 & 15.18 & 0.544 & 23.539 & 0.145 & 0.128 \\
\hline HIP 27628 & 26.73 & 0.081 & 25.936 & 0.071 & 0.066 \\
\hline HIP 27887 & 13.0 & 0.376 & 21.413 & 0.053 & 0.045 \\
\hline HIP 27890 & 26.25 & 0.19 & 24.754 & 0.058 & 0.053 \\
\hline HIP 28103 & 14.88 & 0.2 & 26.143 & 0.282 & 0.266 \\
\hline HIP 28360 & 24.87 & 0.031 & 27.217 & 0.098 & 0.096 \\
\hline HIP 28908 & 25.62 & 0.143 & 22.489 & 0.022 & 0.019 \\
\hline HIP 28954 & 15.27 & 0.327 & 21.575 & 0.046 & 0.039 \\
\hline HIP 29271 & 10.2 & 0.582 & 24.95 & 0.369 & 0.337 \\
\hline HIP 29295 & 5.75 & 0.401 & 20.008 & 0.051 & 0.043 \\
\hline HIP 29525 & 17.95 & 0.337 & 22.3 & 0.055 & 0.048 \\
\hline HIP 29568 & 16.72 & 0.423 & 22.752 & 0.083 & 0.073 \\
\hline HIP 29650 & 20.82 & 0.358 & 24.425 & 0.109 & 0.097 \\
\hline HIP 29800 & 19.25 & 0.394 & 24.751 & 0.145 & 0.131 \\
\hline HIP 29860 & 19.25 & 0.396 & 23.768 & 0.102 & 0.09 \\
\hline HIP 30503 & 21.88 & 0.228 & 22.401 & 0.036 & 0.031 \\
\hline HIP 31592 & 19.75 & 0.225 & 25.568 & 0.163 & 0.151 \\
\hline HIP 32349 & 2.63 & 0.025 & 30.177 & 0.872 & 0.861 \\
\hline HIP 32362 & 18.0 & 0.145 & 26.221 & 0.21 & 0.199 \\
\hline HIP 32366 & 24.38 & 0.207 & 23.238 & 0.041 & 0.036 \\
\hline
\end{tabular}


Table 2 (Continued).

\begin{tabular}{|c|c|c|c|c|c|}
\hline Name & Dist (pc) & Int. time (day) & $\Delta \operatorname{mag}$ & $C_{\text {brown }}$ & $C_{\mathrm{IAC}}$ \\
\hline HIP 32439 & 17.87 & 0.465 & 24.414 & 0.152 & 0.136 \\
\hline HIP 32480 & 16.72 & 0.498 & 24.565 & 0.18 & 0.163 \\
\hline HIP 32607 & 29.6 & 0.051 & 26.098 & 0.046 & 0.043 \\
\hline HIP 32765 & 25.26 & 0.215 & 24.454 & 0.061 & 0.055 \\
\hline HIP 32984 & 8.71 & 0.777 & 22.753 & 0.205 & 0.179 \\
\hline HIP 33094 & 25.89 & 0.154 & 22.824 & 0.026 & 0.023 \\
\hline HIP 33277 & 17.24 & 0.471 & 23.721 & 0.125 & 0.111 \\
\hline HIP 33690 & 18.33 & 0.245 & 21.638 & 0.033 & 0.028 \\
\hline HIP 33817 & 14.65 & 0.425 & 22.086 & 0.071 & 0.061 \\
\hline HIP 34017 & 19.13 & 0.372 & 23.259 & 0.082 & 0.072 \\
\hline HIP 34834 & 21.43 & 0.231 & 25.368 & 0.13 & 0.12 \\
\hline HIP 35136 & 16.89 & 0.503 & 24.178 & 0.156 & 0.139 \\
\hline HIP 35296 A & 14.59 & 0.444 & 22.214 & 0.077 & 0.067 \\
\hline HIP 36366 & 18.05 & 0.245 & 25.626 & 0.197 & 0.183 \\
\hline HIP 36399 & 27.24 & 0.132 & 23.032 & 0.024 & 0.021 \\
\hline HIP 36439 & 20.24 & 0.38 & 24.354 & 0.114 & 0.102 \\
\hline HIP 36795 & 25.3 & 0.184 & 25.172 & 0.076 & 0.069 \\
\hline HIP 37279 & 3.51 & 0.063 & 28.816 & 0.796 & 0.778 \\
\hline HIP 37349 & 14.21 & 0.281 & 21.13 & 0.037 & 0.031 \\
\hline HIP 37606 & 24.67 & 0.233 & 24.476 & 0.067 & 0.06 \\
\hline HIP 37826 & 10.36 & 0.073 & 27.756 & 0.442 & 0.43 \\
\hline HIP 38784 & 17.19 & 0.358 & 22.262 & 0.059 & 0.051 \\
\hline HIP 38908 & 16.2 & 0.532 & 24.279 & 0.174 & 0.155 \\
\hline HIP 39157 & 16.77 & 0.216 & 21.173 & 0.028 & 0.024 \\
\hline HIP 39342 & 17.31 & 0.195 & 21.121 & 0.025 & 0.022 \\
\hline HIP 39757 & 19.48 & 0.083 & 26.621 & 0.184 & 0.177 \\
\hline HIP 39780 & 23.29 & 0.266 & 24.017 & 0.068 & 0.06 \\
\hline HIP 39903 & 19.98 & 0.305 & 25.155 & 0.148 & 0.135 \\
\hline HIP 40035 & 22.38 & 0.292 & 23.955 & 0.075 & 0.066 \\
\hline HIP 40693 & 12.49 & 0.674 & 23.689 & 0.205 & 0.181 \\
\hline HIP 40702 & 19.56 & 0.196 & 25.75 & 0.171 & 0.159 \\
\hline HIP 40706 & 28.63 & 0.124 & 25.177 & 0.045 & 0.041 \\
\hline HIP 40843 & 18.27 & 0.438 & 24.634 & 0.156 & 0.141 \\
\hline HIP 41211 & 26.62 & 0.159 & 23.493 & 0.033 & 0.029 \\
\hline
\end{tabular}


Table 2 (Continued).

\begin{tabular}{|c|c|c|c|c|c|}
\hline Name & Dist (pc) & Int. time (day) & $\Delta \operatorname{mag}$ & $C_{\text {brown }}$ & $C_{\mathrm{IAC}}$ \\
\hline HIP 41484 & 22.25 & 0.216 & 22.368 & 0.033 & 0.029 \\
\hline HIP 41926 & 12.21 & 0.621 & 22.933 & 0.151 & 0.132 \\
\hline HIP 42438 & 14.36 & 0.607 & 24.195 & 0.204 & 0.182 \\
\hline HIP 42808 & 11.14 & 0.631 & 22.574 & 0.141 & 0.123 \\
\hline HIP 43587 & 12.34 & 0.673 & 23.552 & 0.197 & 0.173 \\
\hline HIP 43726 & 17.39 & 0.442 & 23.35 & 0.105 & 0.092 \\
\hline HIP 43797 & 24.15 & 0.229 & 23.68 & 0.052 & 0.046 \\
\hline HIP 44075 & 21.03 & 0.323 & 23.609 & 0.077 & 0.068 \\
\hline HIP 44127 & 14.51 & 0.129 & 26.677 & 0.3 & 0.288 \\
\hline HIP 44143 & 26.42 & 0.182 & 24.346 & 0.049 & 0.044 \\
\hline HIP 44897 & 19.19 & 0.377 & 23.372 & 0.086 & 0.076 \\
\hline HIP 45170 & 20.36 & 0.232 & 21.979 & 0.033 & 0.028 \\
\hline HIP 45333 & 19.57 & 0.397 & 24.602 & 0.134 & 0.12 \\
\hline HIP 45343 & 6.11 & 0.566 & 20.808 & 0.09 & 0.076 \\
\hline HIP 45617 & 17.27 & 0.131 & 20.537 & 0.016 & 0.014 \\
\hline HIP 46509 & 17.33 & 0.342 & 25.261 & 0.201 & 0.184 \\
\hline HIP 46580 & 12.91 & 0.281 & 20.919 & 0.036 & 0.031 \\
\hline HIP 46733 & 23.82 & 0.112 & 25.913 & 0.105 & 0.099 \\
\hline HIP 46843 & 17.79 & 0.161 & 20.93 & 0.021 & 0.018 \\
\hline HIP 46853 & 13.48 & 0.158 & 26.543 & 0.327 & 0.312 \\
\hline HIP 47080 & 11.37 & 0.689 & 24.424 & 0.295 & 0.265 \\
\hline HIP 47592 & 15.01 & 0.457 & 25.025 & 0.241 & 0.22 \\
\hline HIP 48113 & 18.37 & 0.425 & 24.727 & 0.159 & 0.143 \\
\hline HIP 48331 & 11.16 & 0.237 & 20.399 & 0.03 & 0.025 \\
\hline HIP 48833 & 28.15 & 0.136 & 24.138 & 0.034 & 0.031 \\
\hline HIP 49081 & 15.05 & 0.571 & 24.431 & 0.206 & 0.185 \\
\hline HIP 49593 & 28.24 & 0.137 & 25.074 & 0.047 & 0.043 \\
\hline HIP 49669 & 24.31 & 0.029 & 27.69 & 0.106 & 0.105 \\
\hline HIP 49809 & 27.74 & 0.141 & 23.924 & 0.034 & 0.03 \\
\hline HIP 49908 & 4.87 & 0.989 & 22.892 & 0.356 & 0.312 \\
\hline HIP 50075 & 22.85 & 0.198 & 22.331 & 0.03 & 0.026 \\
\hline HIP 50384 & 22.81 & 0.257 & 23.348 & 0.054 & 0.047 \\
\hline HIP 50505 & 20.24 & 0.198 & 21.651 & 0.026 & 0.022 \\
\hline HIP 50564 & 21.37 & 0.309 & 24.921 & 0.119 & 0.108 \\
\hline
\end{tabular}


Table 2 (Continued).

\begin{tabular}{|c|c|c|c|c|c|}
\hline Name & Dist (pc) & Int. time (day) & $\Delta \operatorname{mag}$ & $C_{\text {brown }}$ & $C_{\mathrm{IAC}}$ \\
\hline HIP 50954 & 16.22 & 0.219 & 25.922 & 0.244 & 0.229 \\
\hline HIP 51459 & 12.78 & 0.464 & 25.177 & 0.305 & 0.28 \\
\hline HIP 51502 & 21.5 & 0.333 & 24.57 & 0.105 & 0.094 \\
\hline HIP 51523 & 21.81 & 0.298 & 24.914 & 0.112 & 0.102 \\
\hline HIP 51814 & 26.53 & 0.179 & 24.362 & 0.049 & 0.044 \\
\hline HIP 51933 & 25.08 & 0.195 & 23.413 & 0.04 & 0.035 \\
\hline HIP 53229 & 29.09 & 0.108 & 25.297 & 0.043 & 0.04 \\
\hline HIP 53253 & 29.13 & 0.101 & 25.391 & 0.044 & 0.041 \\
\hline HIP 53721 & 14.06 & 0.509 & 24.905 & 0.257 & 0.234 \\
\hline HIP 53910 & 24.45 & 0.039 & 26.951 & 0.103 & 0.1 \\
\hline HIP 54035 & 2.54 & 1.0 & 21.604 & 0.308 & 0.263 \\
\hline HIP 54182 & 28.99 & 0.125 & 24.696 & 0.037 & 0.033 \\
\hline HIP 54211 & 4.86 & 0.263 & 19.093 & 0.027 & 0.023 \\
\hline HIP 54745 & 21.93 & 0.213 & 22.22 & 0.032 & 0.027 \\
\hline HIP 54872 & 17.91 & 0.074 & 26.903 & 0.219 & 0.212 \\
\hline HIP 55691 A & 13.71 & 0.174 & 20.377 & 0.022 & 0.018 \\
\hline HIP 55779 & 27.22 & 0.158 & 24.189 & 0.041 & 0.037 \\
\hline HIP 56445 & 27.23 & 0.133 & 23.075 & 0.024 & 0.021 \\
\hline HIP 56452 & 9.56 & 0.799 & 24.001 & 0.31 & 0.276 \\
\hline HIP 56802 & 26.73 & 0.158 & 23.596 & 0.034 & 0.03 \\
\hline HIP 56997 & 9.61 & 0.688 & 24.618 & 0.365 & 0.329 \\
\hline HIP 56998 & 12.4 & 0.178 & 20.179 & 0.022 & 0.018 \\
\hline HIP 57443 & 9.22 & 0.55 & 25.115 & 0.413 & 0.378 \\
\hline HIP 57507 & 17.47 & 0.358 & 22.357 & 0.061 & 0.053 \\
\hline HIP 57632 & 11.0 & 0.088 & 27.443 & 0.417 & 0.404 \\
\hline HIP 57757 & 10.93 & 0.259 & 26.148 & 0.398 & 0.374 \\
\hline HIP 57939 & 9.09 & 0.782 & 22.992 & 0.22 & 0.192 \\
\hline HIP 58001 & 25.5 & 0.037 & 26.884 & 0.089 & 0.087 \\
\hline HIP 58345 & 10.16 & 0.549 & 21.707 & 0.093 & 0.08 \\
\hline HIP 58576 & 12.76 & 0.676 & 24.144 & 0.236 & 0.21 \\
\hline HIP 58803 & 25.32 & 0.212 & 24.371 & 0.058 & 0.052 \\
\hline HIP 58910 & 5.52 & 0.78 & 21.563 & 0.167 & 0.143 \\
\hline HIP 59072 & 19.76 & 0.204 & 25.689 & 0.166 & 0.154 \\
\hline HIP 59199 & 14.94 & 0.253 & 25.855 & 0.273 & 0.256 \\
\hline
\end{tabular}


Table 2 (Continued).

\begin{tabular}{|c|c|c|c|c|c|}
\hline Name & Dist (pc) & Int. time (day) & $\Delta \mathrm{mag}$ & $C_{\text {brown }}$ & $C_{\mathrm{IAC}}$ \\
\hline HIP 59750 & 22.35 & 0.244 & 22.816 & 0.043 & 0.038 \\
\hline HIP 59774 & 24.69 & 0.072 & 26.26 & 0.097 & 0.092 \\
\hline HIP 60965 & 26.63 & 0.051 & 26.407 & 0.075 & 0.071 \\
\hline HIP 61053 & 21.78 & 0.261 & 22.834 & 0.047 & 0.041 \\
\hline HIP 61084 & 27.15 & 0.037 & 26.765 & 0.07 & 0.068 \\
\hline HIP 61174 & 18.28 & 0.271 & 25.475 & 0.188 & 0.174 \\
\hline HIP 61291 & 16.18 & 0.248 & 21.274 & 0.033 & 0.028 \\
\hline HIP 61317 & 8.44 & 0.386 & 25.755 & 0.475 & 0.442 \\
\hline HIP 62145 & 14.88 & 0.318 & 21.442 & 0.043 & 0.037 \\
\hline HIP 62207 & 17.38 & 0.456 & 23.567 & 0.116 & 0.102 \\
\hline HIP 62523 & 16.93 & 0.418 & 22.789 & 0.083 & 0.073 \\
\hline HIP 62951 & 7.53 & 0.378 & 20.357 & 0.049 & 0.041 \\
\hline HIP 62956 & 25.31 & 0.03 & 27.46 & 0.093 & 0.091 \\
\hline HIP 63076 & 29.29 & 0.112 & 24.085 & 0.028 & 0.025 \\
\hline HIP 63613 & 27.87 & 0.107 & 25.502 & 0.055 & 0.051 \\
\hline HIP 63721 & 4.62 & 0.538 & 20.255 & 0.081 & 0.068 \\
\hline HIP 64394 & 9.13 & 0.381 & 25.732 & 0.447 & 0.416 \\
\hline HIP 64408 & 20.67 & 0.337 & 24.857 & 0.127 & 0.115 \\
\hline HIP 64583 & 18.2 & 0.38 & 25.015 & 0.174 & 0.159 \\
\hline HIP 64792 & 17.56 & 0.467 & 24.586 & 0.166 & 0.15 \\
\hline HIP 64924 & 8.56 & 0.553 & 25.147 & 0.439 & 0.403 \\
\hline HIP 65109 & 18.02 & 0.076 & 26.859 & 0.216 & 0.209 \\
\hline HIP 65352 & 15.45 & 0.25 & 21.164 & 0.033 & 0.028 \\
\hline HIP 65355 & 16.14 & 0.162 & 20.661 & 0.021 & 0.017 \\
\hline HIP 65530 & 21.17 & 0.228 & 22.163 & 0.034 & 0.029 \\
\hline HIP 65721 & 17.99 & 0.426 & 24.802 & 0.169 & 0.153 \\
\hline HIP 66249 & 22.71 & 0.095 & 26.18 & 0.124 & 0.117 \\
\hline HIP 66765 & 15.65 & 0.293 & 21.439 & 0.039 & 0.034 \\
\hline HIP 67153 & 19.4 & 0.236 & 25.54 & 0.169 & 0.157 \\
\hline HIP 67155 & 5.41 & 0.319 & 19.535 & 0.036 & 0.03 \\
\hline HIP 67275 & 15.62 & 0.345 & 25.386 & 0.242 & 0.224 \\
\hline HIP 67422 A & 13.41 & 0.362 & 21.413 & 0.05 & 0.043 \\
\hline HIP 67927 & 11.4 & 0.139 & 26.884 & 0.397 & 0.38 \\
\hline HIP 68030 & 24.86 & 0.165 & 22.587 & 0.026 & 0.023 \\
\hline
\end{tabular}


Table 2 (Continued).

\begin{tabular}{|c|c|c|c|c|c|}
\hline Name & Dist (pc) & Int. time (day) & $\Delta \operatorname{mag}$ & $C_{\text {brown }}$ & $C_{\mathrm{IAC}}$ \\
\hline HIP 68184 & 10.06 & 0.729 & 22.959 & 0.194 & 0.169 \\
\hline HIP 68682 & 16.98 & 0.42 & 22.833 & 0.085 & 0.074 \\
\hline HIP 68933 & 18.03 & 0.072 & 26.912 & 0.216 & 0.209 \\
\hline HIP 69671 & 21.22 & 0.258 & 22.541 & 0.043 & 0.037 \\
\hline HIP 69701 & 22.24 & 0.194 & 25.491 & 0.121 & 0.112 \\
\hline HIP 69713 & 29.07 & 0.124 & 24.773 & 0.037 & 0.034 \\
\hline HIP 69965 & 18.03 & 0.43 & 23.529 & 0.106 & 0.093 \\
\hline HIP 69972 & 11.8 & 0.592 & 22.482 & 0.124 & 0.108 \\
\hline HIP 69989 & 26.1 & 0.181 & 23.903 & 0.043 & 0.038 \\
\hline HIP 70319 & 17.19 & 0.411 & 22.815 & 0.082 & 0.072 \\
\hline HIP 70497 & 14.53 & 0.261 & 25.85 & 0.284 & 0.266 \\
\hline HIP 70857 & 19.89 & 0.178 & 21.426 & 0.023 & 0.02 \\
\hline HIP 70873 & 23.74 & 0.172 & 22.267 & 0.025 & 0.022 \\
\hline HIP 71075 & 26.61 & 0.056 & 26.311 & 0.074 & 0.071 \\
\hline HIP 71181 & 13.22 & 0.337 & 21.254 & 0.046 & 0.039 \\
\hline HIP 71284 & 15.83 & 0.314 & 25.495 & 0.241 & 0.224 \\
\hline HIP 71855 & 20.0 & 0.203 & 21.649 & 0.027 & 0.023 \\
\hline HIP 71957 & 18.27 & 0.204 & 25.823 & 0.197 & 0.185 \\
\hline HIP 72567 & 18.17 & 0.429 & 23.644 & 0.109 & 0.097 \\
\hline HIP 72603 & 22.98 & 0.285 & 24.398 & 0.082 & 0.073 \\
\hline HIP 72622 & 23.24 & 0.062 & 26.537 & 0.119 & 0.114 \\
\hline HIP 72848 & 11.51 & 0.715 & 23.602 & 0.219 & 0.193 \\
\hline HIP 73100 & 25.11 & 0.202 & 23.679 & 0.046 & 0.04 \\
\hline HIP 73165 & 26.9 & 0.165 & 25.043 & 0.058 & 0.052 \\
\hline HIP 73996 & 19.55 & 0.347 & 25.001 & 0.15 & 0.136 \\
\hline HIP 74273 & 24.2 & 0.167 & 22.366 & 0.025 & 0.022 \\
\hline HIP 74537 & 17.67 & 0.311 & 21.982 & 0.046 & 0.04 \\
\hline HIP 74605 & 25.34 & 0.211 & 24.386 & 0.059 & 0.053 \\
\hline HIP 74702 & 15.85 & 0.274 & 21.367 & 0.036 & 0.031 \\
\hline HIP 74975 & 25.38 & 0.211 & 24.352 & 0.058 & 0.052 \\
\hline HIP 75181 & 14.81 & 0.589 & 24.092 & 0.188 & 0.167 \\
\hline HIP 75718 & 20.58 & 0.139 & 21.214 & 0.018 & 0.015 \\
\hline HIP 75809 & 21.85 & 0.176 & 21.803 & 0.024 & 0.02 \\
\hline HIP 76219 & 28.93 & 0.123 & 24.381 & 0.033 & 0.03 \\
\hline
\end{tabular}


Table 2 (Continued).

\begin{tabular}{|c|c|c|c|c|c|}
\hline Name & Dist (pc) & Int. time (day) & $\Delta \operatorname{mag}$ & $C_{\text {brown }}$ & $C_{\mathrm{IAC}}$ \\
\hline HIP 76267 & 23.01 & 0.038 & 27.087 & 0.125 & 0.121 \\
\hline HIP 76829 & 17.44 & 0.34 & 25.26 & 0.198 & 0.182 \\
\hline HIP 77052 & 14.66 & 0.578 & 23.722 & 0.165 & 0.146 \\
\hline HIP 77070 & 22.68 & 0.084 & 26.306 & 0.125 & 0.119 \\
\hline HIP 77257 & 12.12 & 0.385 & 25.5 & 0.338 & 0.313 \\
\hline HIP 77358 & 15.35 & 0.525 & 23.372 & 0.132 & 0.117 \\
\hline HIP 77760 & 15.89 & 0.352 & 25.336 & 0.234 & 0.216 \\
\hline HIP 77801 & 17.35 & 0.437 & 23.278 & 0.102 & 0.089 \\
\hline HIP 77952 & 12.38 & 0.126 & 26.908 & 0.365 & 0.351 \\
\hline HIP 78072 & 11.25 & 0.272 & 26.058 & 0.385 & 0.361 \\
\hline HIP 78459 & 17.24 & 0.488 & 24.429 & 0.164 & 0.147 \\
\hline HIP 78527 & 21.03 & 0.173 & 25.748 & 0.145 & 0.135 \\
\hline HIP 78775 & 14.52 & 0.451 & 22.244 & 0.079 & 0.069 \\
\hline HIP 79190 & 14.67 & 0.304 & 21.331 & 0.041 & 0.035 \\
\hline HIP 79248 & 17.57 & 0.328 & 22.113 & 0.051 & 0.044 \\
\hline HIP 79537 & 13.89 & 0.226 & 20.787 & 0.029 & 0.025 \\
\hline HIP 79578 & 21.55 & 0.168 & 21.662 & 0.022 & 0.019 \\
\hline HIP 79672 & 13.9 & 0.625 & 24.242 & 0.217 & 0.194 \\
\hline HIP 79822 & 29.74 & 0.107 & 24.346 & 0.028 & 0.026 \\
\hline HIP 80179 & 27.27 & 0.165 & 24.698 & 0.049 & 0.044 \\
\hline HIP 80331 & 28.23 & 0.053 & 26.202 & 0.058 & 0.055 \\
\hline HIP 80337 & 12.78 & 0.641 & 24.472 & 0.261 & 0.235 \\
\hline HIP 80686 & 12.12 & 0.492 & 25.116 & 0.32 & 0.293 \\
\hline HIP 81300 & 9.75 & 0.794 & 23.977 & 0.302 & 0.268 \\
\hline HIP 81935 & 14.26 & 0.166 & 20.386 & 0.021 & 0.017 \\
\hline HIP 82003 & 9.81 & 0.179 & 19.732 & 0.021 & 0.017 \\
\hline HIP 82020 & 26.11 & 0.192 & 24.816 & 0.061 & 0.055 \\
\hline HIP 82396 & 19.54 & 0.085 & 26.601 & 0.183 & 0.175 \\
\hline HIP 82587 & 29.19 & 0.111 & 23.885 & 0.026 & 0.023 \\
\hline HIP 82588 & 17.25 & 0.303 & 21.827 & 0.043 & 0.037 \\
\hline HIP 82621 & 26.94 & 0.13 & 22.785 & 0.021 & 0.019 \\
\hline HIP 82860 & 15.26 & 0.419 & 25.138 & 0.241 & 0.22 \\
\hline HIP 83000 & 28.04 & 0.084 & 25.743 & 0.056 & 0.052 \\
\hline HIP 83389 & 18.59 & 0.25 & 21.73 & 0.034 & 0.029 \\
\hline
\end{tabular}


Table 2 (Continued).

\begin{tabular}{|c|c|c|c|c|c|}
\hline Name & Dist (pc) & Int. time (day) & $\Delta$ mag & $C_{\text {brown }}$ & $C_{\mathrm{IAC}}$ \\
\hline HIP 83541 & 17.84 & 0.284 & 21.815 & 0.04 & 0.034 \\
\hline HIP 83591 & 10.71 & 0.218 & 20.197 & 0.027 & 0.023 \\
\hline HIP 83601 & 20.67 & 0.317 & 23.214 & 0.067 & 0.058 \\
\hline HIP 83609 & 5.62 & 0.311 & 19.561 & 0.035 & 0.029 \\
\hline HIP 83990 & 13.62 & 0.26 & 20.929 & 0.034 & 0.029 \\
\hline HIP 84143 & 22.53 & 0.104 & 26.117 & 0.126 & 0.119 \\
\hline HIP 84478 & 5.95 & 0.95 & 23.071 & 0.332 & 0.29 \\
\hline HIP 84862 & 14.33 & 0.578 & 24.57 & 0.231 & 0.208 \\
\hline HIP 85042 & 19.52 & 0.316 & 22.628 & 0.056 & 0.049 \\
\hline HIP 85235 & 12.8 & 0.588 & 22.853 & 0.136 & 0.119 \\
\hline HIP 85295 & 7.7 & 0.45 & 20.704 & 0.063 & 0.053 \\
\hline HIP 86201 & 23.16 & 0.251 & 25.007 & 0.097 & 0.088 \\
\hline HIP 86400 & 11.0 & 0.644 & 22.624 & 0.147 & 0.128 \\
\hline HIP 86486 & 21.45 & 0.29 & 25.037 & 0.121 & 0.11 \\
\hline HIP 86614 & 22.84 & 0.226 & 25.235 & 0.107 & 0.098 \\
\hline HIP 86620 & 23.06 & 0.257 & 23.538 & 0.057 & 0.05 \\
\hline HIP 86742 & 25.09 & 0.079 & 26.132 & 0.091 & 0.086 \\
\hline HIP 86796 & 15.51 & 0.512 & 24.744 & 0.215 & 0.195 \\
\hline HIP 86974 & 8.31 & 0.253 & 26.393 & 0.501 & 0.474 \\
\hline HIP 88175 & 23.55 & 0.233 & 25.073 & 0.094 & 0.085 \\
\hline HIP 88635 & 29.7 & 0.065 & 25.833 & 0.044 & 0.041 \\
\hline HIP 88694 & 17.55 & 0.439 & 23.382 & 0.104 & 0.092 \\
\hline HIP 88771 & 26.63 & 0.088 & 25.85 & 0.071 & 0.066 \\
\hline HIP 88972 & 11.02 & 0.686 & 23.01 & 0.178 & 0.156 \\
\hline HIP 89042 & 17.61 & 0.476 & 24.3 & 0.15 & 0.135 \\
\hline HIP 89348 & 22.92 & 0.28 & 24.79 & 0.094 & 0.085 \\
\hline HIP 89474 & 22.82 & 0.213 & 22.54 & 0.034 & 0.03 \\
\hline HIP 89962 & 18.54 & 0.154 & 26.107 & 0.197 & 0.186 \\
\hline HIP 90496 & 23.97 & 0.088 & 26.129 & 0.105 & 0.099 \\
\hline HIP 90790 & 13.25 & 0.399 & 21.587 & 0.059 & 0.05 \\
\hline HIP 91262 & 7.68 & 0.03 & 29.135 & 0.559 & 0.549 \\
\hline HIP 91768 & 3.52 & 0.41 & 19.289 & 0.05 & 0.041 \\
\hline HIP 92024 & 28.55 & 0.136 & 24.804 & 0.041 & 0.037 \\
\hline HIP 92043 & 19.21 & 0.229 & 25.603 & 0.174 & 0.162 \\
\hline
\end{tabular}


Table 2 (Continued).

\begin{tabular}{|c|c|c|c|c|c|}
\hline Name & Dist $(\mathrm{pc})$ & Int. time (day) & $\Delta$ mag & $C_{\text {brown }}$ & $C_{\mathrm{IAC}}$ \\
\hline HIP 92161 & 28.89 & 0.115 & 25.259 & 0.044 & 0.041 \\
\hline HIP 92549 & 25.67 & 0.187 & 23.697 & 0.042 & 0.037 \\
\hline HIP 93747 & 25.46 & 0.055 & 26.449 & 0.088 & 0.084 \\
\hline HIP 93858 & 16.95 & 0.437 & 23.018 & 0.094 & 0.082 \\
\hline HIP 93966 & 21.43 & 0.285 & 23.049 & 0.056 & 0.049 \\
\hline HIP 94083 & 27.3 & 0.159 & 24.403 & 0.044 & 0.039 \\
\hline HIP 94376 & 29.87 & 0.06 & 25.889 & 0.043 & 0.04 \\
\hline HIP 95149 & 18.83 & 0.293 & 22.147 & 0.045 & 0.039 \\
\hline HIP 95319 & 15.76 & 0.465 & 22.822 & 0.097 & 0.085 \\
\hline HIP 95447 & 15.18 & 0.537 & 24.662 & 0.217 & 0.196 \\
\hline HIP 95501 & 15.53 & 0.158 & 26.357 & 0.269 & 0.256 \\
\hline HIP 95995 A & 16.96 & 0.367 & 22.278 & 0.061 & 0.053 \\
\hline HIP 96100 & 5.75 & 0.532 & 25.374 & 0.576 & 0.531 \\
\hline HIP 96258 & 25.11 & 0.198 & 23.568 & 0.043 & 0.038 \\
\hline HIP 96441 & 18.34 & 0.281 & 25.424 & 0.186 & 0.172 \\
\hline HIP 96895 & 21.08 & 0.315 & 23.431 & 0.07 & 0.062 \\
\hline HIP 96901 & 21.21 & 0.284 & 22.947 & 0.054 & 0.047 \\
\hline HIP 97649 & 5.12 & 0.061 & 28.669 & 0.689 & 0.673 \\
\hline HIP 97650 & 27.87 & 0.135 & 23.712 & 0.03 & 0.027 \\
\hline HIP 97675 & 19.19 & 0.403 & 24.679 & 0.143 & 0.129 \\
\hline HIP 97944 & 14.05 & 0.552 & 23.011 & 0.128 & 0.113 \\
\hline HIP 98036 & 13.7 & 0.257 & 25.942 & 0.308 & 0.289 \\
\hline HIP 98066 & 25.99 & 0.196 & 24.561 & 0.057 & 0.051 \\
\hline HIP 98470 & 21.25 & 0.322 & 23.721 & 0.078 & 0.069 \\
\hline HIP 98698 & 12.86 & 0.225 & 20.606 & 0.029 & 0.024 \\
\hline HIP 98767 & 15.86 & 0.539 & 23.947 & 0.159 & 0.142 \\
\hline HIP 98792 & 15.77 & 0.222 & 21.051 & 0.029 & 0.025 \\
\hline HIP 98819 & 17.77 & 0.453 & 23.791 & 0.122 & 0.108 \\
\hline HIP 98921 & 18.79 & 0.371 & 23.036 & 0.076 & 0.067 \\
\hline HIP 98959 & 17.73 & 0.425 & 23.298 & 0.098 & 0.086 \\
\hline HIP 99031 & 23.95 & 0.242 & 23.966 & 0.061 & 0.054 \\
\hline HIP 99137 & 23.43 & 0.197 & 22.533 & 0.031 & 0.027 \\
\hline HIP 99240 & 6.11 & 0.299 & 26.312 & 0.598 & 0.564 \\
\hline HIP 99461 & 6.01 & 0.782 & 24.537 & 0.499 & 0.452 \\
\hline
\end{tabular}


Table 2 (Continued).

\begin{tabular}{|c|c|c|c|c|c|}
\hline Name & Dist (pc) & Int. time (day) & $\Delta$ mag & $C_{\text {brown }}$ & $C_{\mathrm{IAC}}$ \\
\hline HIP 99572 & 27.7 & 0.118 & 22.892 & 0.02 & 0.018 \\
\hline HIP 99701 & 6.2 & 0.402 & 20.137 & 0.052 & 0.044 \\
\hline HIP 99825 & 8.91 & 0.825 & 23.923 & 0.323 & 0.287 \\
\hline HIP 100017 & 17.57 & 0.453 & 23.653 & 0.117 & 0.104 \\
\hline HIP 100511 & 26.25 & 0.131 & 22.509 & 0.02 & 0.018 \\
\hline HIP 100925 & 19.52 & 0.216 & 21.657 & 0.029 & 0.025 \\
\hline HIP 101345 & 24.4 & 0.212 & 23.393 & 0.044 & 0.039 \\
\hline HIP 101612 & 27.79 & 0.153 & 24.797 & 0.046 & 0.042 \\
\hline HIP 101983 & 24.66 & 0.232 & 24.425 & 0.066 & 0.059 \\
\hline HIP 101997 & 14.38 & 0.499 & 22.622 & 0.101 & 0.088 \\
\hline HIP 102040 & 20.95 & 0.242 & 22.254 & 0.037 & 0.032 \\
\hline HIP 102333 & 24.17 & 0.195 & 25.258 & 0.09 & 0.083 \\
\hline HIP 102422 & 14.27 & 0.201 & 26.191 & 0.299 & 0.282 \\
\hline HIP 102485 & 14.68 & 0.297 & 25.672 & 0.275 & 0.256 \\
\hline HIP 102488 & 22.29 & 0.068 & 26.555 & 0.133 & 0.128 \\
\hline HIP 103389 & 21.97 & 0.291 & 23.579 & 0.067 & 0.059 \\
\hline HIP 103458 & 22.14 & 0.176 & 21.863 & 0.024 & 0.02 \\
\hline HIP 103673 & 28.01 & 0.12 & 23.163 & 0.022 & 0.02 \\
\hline HIP 104214 & 3.5 & 0.778 & 24.704 & 0.657 & 0.599 \\
\hline HIP 104217 & 3.5 & 0.966 & 23.852 & 0.562 & 0.503 \\
\hline HIP 104239 A & 17.57 & 0.13 & 20.584 & 0.016 & 0.014 \\
\hline HIP 105090 & 3.95 & 1.019 & 22.792 & 0.39 & 0.341 \\
\hline HIP 105199 & 15.04 & 0.077 & 27.164 & 0.291 & 0.282 \\
\hline HIP 105858 & 9.26 & 0.361 & 25.804 & 0.445 & 0.415 \\
\hline HIP 106440 & 4.95 & 0.288 & 19.245 & 0.031 & 0.026 \\
\hline HIP 106559 & 27.1 & 0.14 & 23.208 & 0.027 & 0.023 \\
\hline HIP 106696 & 14.62 & 0.283 & 21.212 & 0.037 & 0.032 \\
\hline HIP 107089 & 21.2 & 0.174 & 25.728 & 0.142 & 0.132 \\
\hline HIP 107350 & 17.89 & 0.43 & 23.466 & 0.104 & 0.092 \\
\hline HIP 107556 & 11.87 & 0.142 & 26.818 & 0.38 & 0.364 \\
\hline HIP 107649 & 15.99 & 0.539 & 24.202 & 0.173 & 0.154 \\
\hline HIP 107975 & 27.45 & 0.143 & 23.663 & 0.031 & 0.028 \\
\hline HIP 108036 & 26.62 & 0.173 & 24.239 & 0.046 & 0.041 \\
\hline HIP 108870 & 3.62 & 0.625 & 25.183 & 0.688 & 0.633 \\
\hline
\end{tabular}


Table 2 (Continued).

\begin{tabular}{|c|c|c|c|c|c|}
\hline Name & Dist (pc) & Int. time (day) & $\Delta \operatorname{mag}$ & $C_{\text {brown }}$ & $C_{\mathrm{IAC}}$ \\
\hline HIP 109176 & 11.73 & 0.252 & 26.12 & 0.371 & 0.349 \\
\hline HIP 109378 & 21.56 & 0.164 & 21.628 & 0.022 & 0.018 \\
\hline HIP 109422 & 18.28 & 0.399 & 24.905 & 0.168 & 0.153 \\
\hline HIP 109427 & 28.3 & 0.071 & 25.894 & 0.055 & 0.052 \\
\hline HIP 109821 & 22.05 & 0.24 & 22.624 & 0.04 & 0.035 \\
\hline HIP 110649 & 20.56 & 0.369 & 24.363 & 0.11 & 0.098 \\
\hline HIP 111449 & 22.68 & 0.294 & 24.314 & 0.083 & 0.074 \\
\hline HIP 112117 & 23.64 & 0.22 & 23.07 & 0.042 & 0.037 \\
\hline HIP 112447 & 16.3 & 0.286 & 25.586 & 0.233 & 0.217 \\
\hline HIP 112935 & 27.28 & 0.155 & 24.059 & 0.038 & 0.034 \\
\hline HIP 113137 & 26.75 & 0.131 & 22.721 & 0.021 & 0.019 \\
\hline HIP 113283 & 7.61 & 0.849 & 22.821 & 0.242 & 0.211 \\
\hline HIP 113357 & 15.61 & 0.554 & 24.306 & 0.186 & 0.167 \\
\hline HIP 113368 & 7.7 & 0.065 & 28.225 & 0.554 & 0.54 \\
\hline HIP 113421 & 19.86 & 0.31 & 22.688 & 0.055 & 0.048 \\
\hline HIP 113576 & 8.22 & 0.273 & 20.018 & 0.033 & 0.028 \\
\hline HIP 113860 & 29.42 & 0.109 & 24.09 & 0.027 & 0.024 \\
\hline HIP 114046 & 3.28 & 1.0 & 21.937 & 0.308 & 0.265 \\
\hline HIP 114430 & 27.65 & 0.123 & 23.011 & 0.022 & 0.019 \\
\hline HIP 114570 & 24.59 & 0.192 & 25.221 & 0.085 & 0.077 \\
\hline HIP 114622 & 6.54 & 0.811 & 24.377 & 0.46 & 0.415 \\
\hline HIP 114924 & 20.5 & 0.362 & 24.042 & 0.098 & 0.087 \\
\hline HIP 114948 & 20.54 & 0.356 & 23.94 & 0.094 & 0.083 \\
\hline HIP 114996 & 23.06 & 0.154 & 25.676 & 0.112 & 0.104 \\
\hline HIP 116085 & 16.93 & 0.296 & 21.711 & 0.041 & 0.036 \\
\hline HIP 116584 & 26.41 & 0.133 & 25.433 & 0.068 & 0.063 \\
\hline HIP 116727 & 14.1 & 0.186 & 26.3 & 0.305 & 0.29 \\
\hline HIP 116745 & 11.42 & 0.44 & 21.427 & 0.065 & 0.056 \\
\hline HIP 116771 & 13.71 & 0.317 & 25.66 & 0.3 & 0.279 \\
\hline HIP 120005 & 6.11 & 0.543 & 20.704 & 0.083 & 0.07 \\
\hline
\end{tabular}




\section{Acknowledgments}

This work was funded by the Science Investigation Team of the Nancy Grace Roman Space Telescope under NASA (Grant No. NNX15AB40G). This work was an expansion of the work done in Ref. 20.

\section{References}

1. D. R. Keithly et al., "Optimal scheduling of exoplanet direct imaging single-visit observations of a blind search survey," J. Astron. Telesc. Instrum. Syst. 6, 027001 (2020).

2. R. A. Brown, "Single-visit photometric and obscurational completeness," Astrophys. J. 624, 1010-1024 (2005).

3. D. Savransky, C. Delacroix, and D. Garrett, "EXOSIMS: exoplanet open-source imaging mission simulator," Astrophysics Source Code Library, record ascl:1706.010 (2017).

4. R. K. Kopparapu et al., "Exoplanet classification and yield estimates for direct imaging missions," Astrophys. J. 856, 122 (2018).

5. D. Garrett, "Exoplanet direct imaging detection metrics and exoplanet popu- lations," $\mathrm{PhD}$ Dissertation, Cornell University (2018).

6. D. Garrett and D. Savransky, "Analytical formulation of the single-visit completeness joint probability density function," Astrophys. J. 828, 20 (2016).

7. N. J. Kasdin et al., "The Nancy Grace Roman Space Telescope Coronagraph Instrument (CGI) technology demonstration," Proc. SPIE 11443, 114431U (2020).

8. Science and Technology Definition Team, "HabEx final report," Tech. Rep., Jet Propulsion Laboratory (2019).

9. Science and Technology Definition Team, "LUVOIR final report," Tech. Rep., NASA (2019).

10. R. Morgan et al., "Exoplanet yield estimation for decadal study concepts using EX," in 227th Meeting Am. Astron. Soc. (2020).

11. R. Morgan et al., "The standard definitions and evaluation team final report a common comparison of exoplanet yield," Tech. Rep. (2019).

12. Jet Propulsion Laboratory, "Habitable exoplanet observatory final report," Tech. Rep. (2019).

13. R. A. Brown and R. Soummer, "New completeness methods for estimating exoplanet discoveries by direct detection," Astrophys. J. 715, 122-131 (2010).

14. B. Spain, Analytical Conics, Dover, London (2007).

15. W. H. Besant, Conic Sections, Treated Geometrically, 9th ed., London (1895).

16. T. A. A. Broadbent, L. Kuipers, and R. Timman, Handbook of Mathematics, Vol. 54, 5th ed., Springer, Berlin (1970).

17. E. Agol, "Rounding up the wanderers: optimizing coronagraphic searches for extrasolar planets," Mon. Not. R. Astron. Soc. 374(4), 1271-1289 (2007).

18. D. Savransky, E. Cady, and N. J. Kasdin, "Parameter distributions of Keplerian orbiTS," Astrophys. J. 728(1), 66 (2011)

19. M. Abramowitz and I. A. Stegun, Handbook of Mathemaical Functions with Formulas, Graphsm and Mathematical Tables, 9th ed., Dover (1972).

20. D. Keithly and D. Savransky, "Integration time adjusted completeness," Proc. SPIE 11443, 1144324 (2020).

Dean Keithly is a PhD candidate in the Sibley School of Mechanical and Aerospace Engineering at Cornell University. He received his bachelor's degree in mechanical engineering from Michigan Technological University, where he built the Oculus-ASR satellite thermal control subsystem. He received his master's degree in systems engineering from Cornell University, working on projects funded by the NASA Institute of Advanced Concepts. His primary research focus is on spacecraft modeling, mission modeling, optimization, and scheduling.

Dmitry Savransky is a professor in the Sibley School of Mechanical and Aerospace Engineering at Cornell University and PI of the Space Imaging and Optical Systems 
Laboratory. He received his $\mathrm{PhD}$ from Princeton University in 2011 and was a postdoctoral researcher at Lawrence Livermore National Laboratory, where he worked on integration, testing and commissioning of the Gemini Planet Imager. His research focuses on the optimization of ground and space-based exoplanet imaging surveys, control of autonomous optical systems, and advanced image processing.

Corey Spohn received a Bachelor of Science in both physics and engineering science and mechanics from Virginia Tech in May 2018. During his time at Virginia Tech he focused heavily on astrophysics while conducting research on anti-frosting surfaces, inter-droplet ice bridging, and flying snakes. For his senior design project, he investigated human-robot dynamics in a crowded panic situation by having humans play the children's game sharks and minnows with robots in a motion capture studio. He began working towards a $\mathrm{PhD}$ in the fall of 2018 and joined the Space Imaging and Optical Systems Lab in December 2018 where he is currently researching exoplanet mission scheduling. 